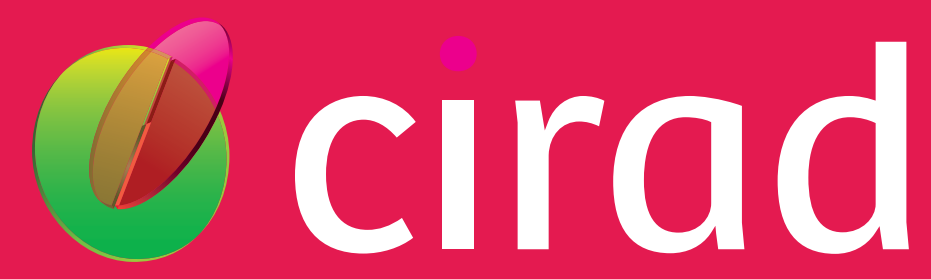

LARECHERCHE AGRONOMIQUE POUR LE DÉVELOPPEMENT
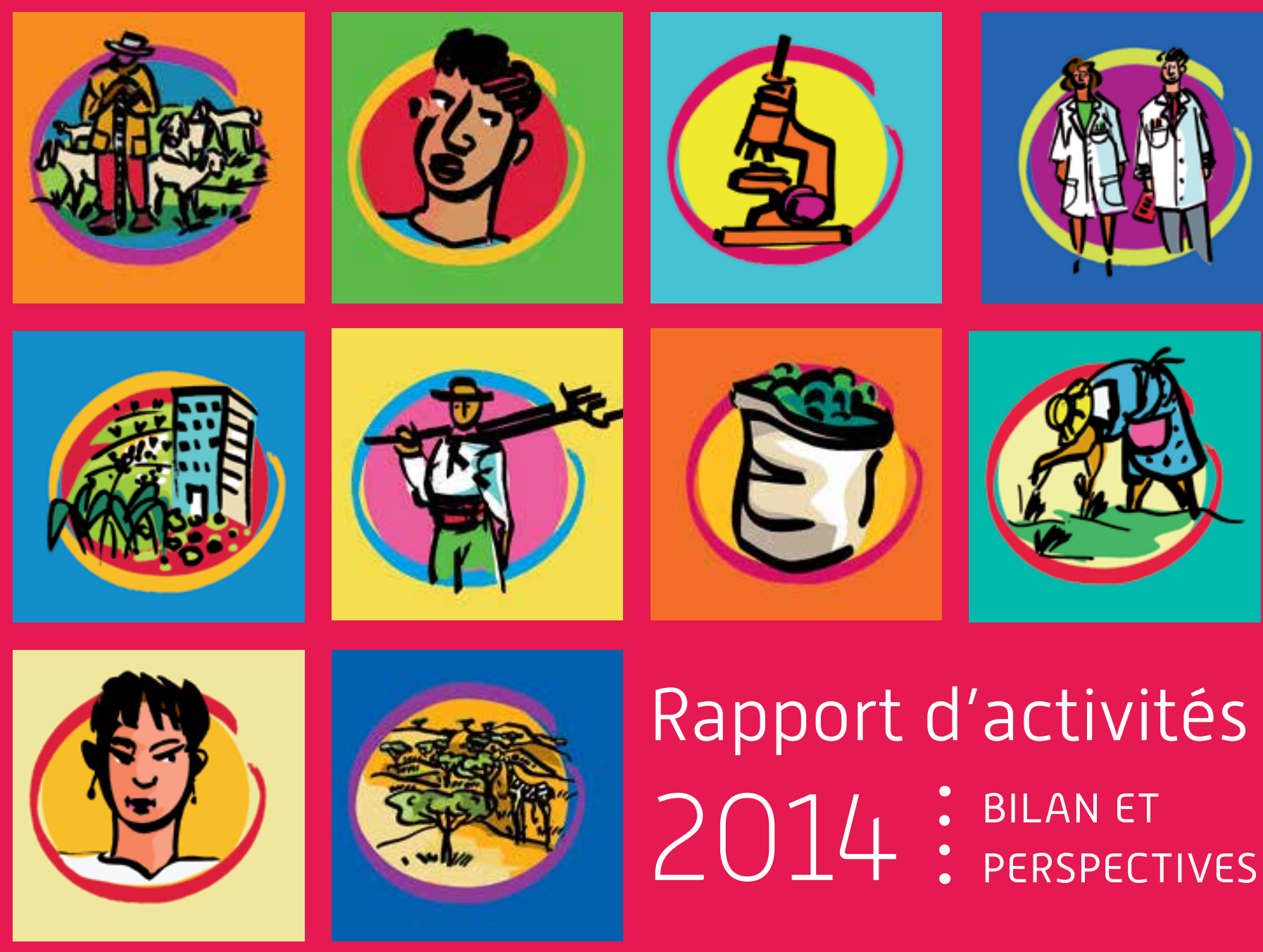

\title{
Rapport d'activités
}
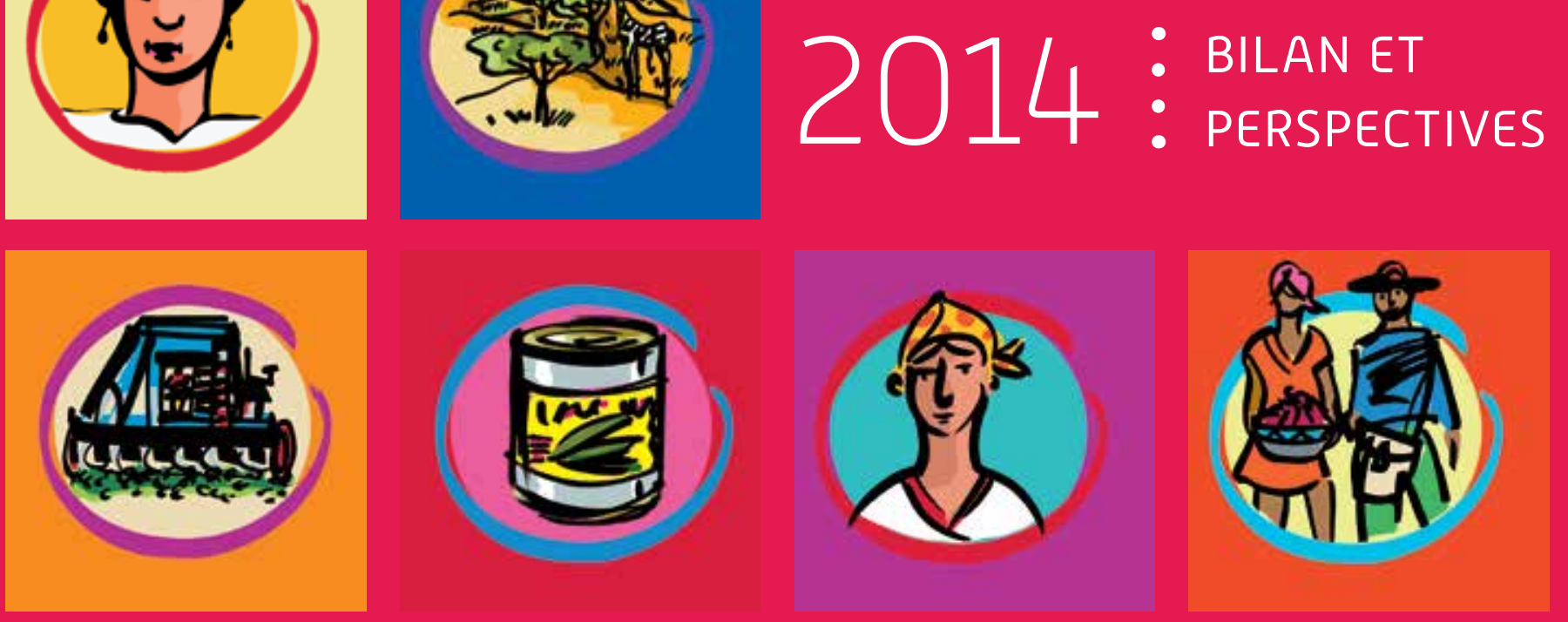


\section{changement climatique}

\section{biodiversité}

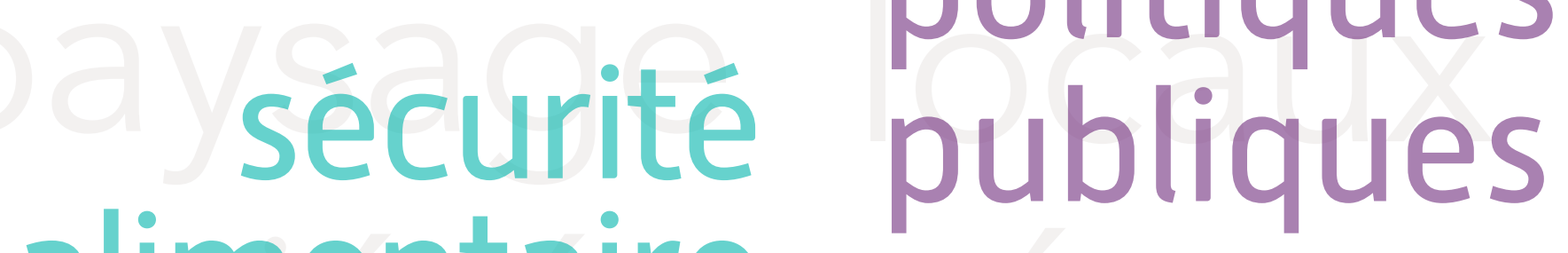

alimentaire

\section{politiques \\ publiques}

\section{agroécologie}

\section{agricultures \\ fanitiales}

$$
\begin{aligned}
& \text { maladies } \\
& \text { émergentes }
\end{aligned}
$$




\section{Le mot de Michel Eddi,}

\section{président directeur général du Cirad}

Les Nations unies ont déclaré l'année 2014 « Année internationale de l'agriculture familiale ». Le Cirad pouvait-il rêver d'un thème plus opportun pour apporter sa contribution à la somme des réflexions, interrogations et débats qui traversent la société internationale sur ce thème crucial pour l'avenir des agricultures du monde ? Sous le parrainage des ministres en charge de l'Agriculture et du Développement, ce fut l'occasion pour nous de faire connaitre les résultats issus de nos propres travaux ainsi que l'expertise développée à ce sujet, depuis des décennies, sur un enjeu qui reste au centre de l'avenir des agricultures du Nord et du Sud. Parce que l'amélioration des conditions de production et de vie des agricultures familiales est au cœur de nos missions, nous avons tout naturellement choisi, nous aussi, d'en faire le sujet de notre thématique annuelle.

Pour cela, nous nous sommes efforcés de participer aux débats et à la réflexion, en chacune des circonstances très diverses offertes au cours de cette année riche en évènements...

- Des rencontres internationales sur le thème « Agricultures familiales et recherche ", organisées à Montpellier par Agropolis international, le GFAR, le CGIAR et le FRM .

- Des échanges interactifs et de qualité avec le grand public, au plus haut point concerné par ce sujet, pendant Salon international de l'agriculture. Cet intérêt traduit les interrogations mais aussi les attentes du plus grand nombre à l'égard du devenir et des capacités d'adaptation des agricultures et de leur organisation millénaire, au Nord comme au Sud, face aux défıs inédits du monde qui vient.

- Le succès de notre exposition itinérante sur ce thème, conçue avec l'AFD, I'Institut français et l'IRD.

- Des publications diverses, sur les multiples aspects de cette réflexion, auxquelles nos collègues ont contribué tout au long de l'année et dont le point d'orgue a été la parution de l'ouvrage collaboratif « Agricultures familiales et mondes à venir » qui s'emploie à résumer pas moins de trois décennies de recherches sur ce sujet.

De belles occasions de reconnaissance et de notoriété pour le Cirad!
2014, c'est aussi, malgré les rigueurs et les difficultés, d'autres circonstances heureuses, d'autre résultats, d'autres raisons de croire en l'avenir.

Je citerai pêle-mêle :

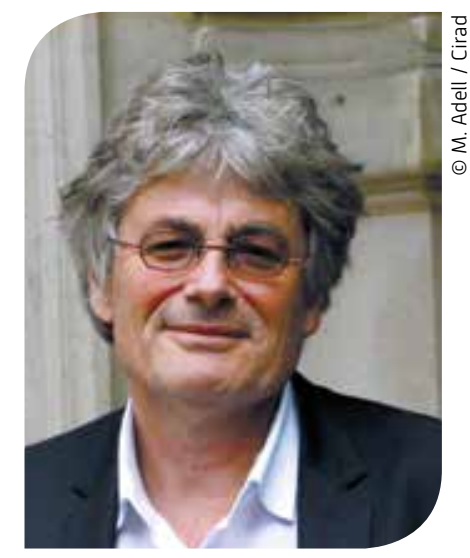

- la signature de notre nou-

veau contrat d'objectifs avec l'Etat dans un cadre de confiance renouvelé ;

- I'adoption par le CA de notre document « Objectifs de stratégie scientifique et partenariale » [OSSP] et la contractualisation de nos objectifs partagés pour cinq ans avec l'ensemble de nos unités de recherches;

- le lancement de notre dynamique de relance économique qui confirme nos capacités structurelles d'adaptation grâce à la mobilisation des collectifs ;

- la consolidation de nos partenariats par la structuration et la pérennité de nos dispositifs de recherche ;

- le renouveau de nos relations avec des pays comme la Côte d'Ivoire, chers à notre histoire commune ;

- de nouvelles initiatives pour l'Afrique, comme «ProlntensAfrica » acceptée par la Commission européenne ;

- des contributions scientifiques partagées avec l'Inra en faveur du concept d'agroécologie porté par le ministre en charge de l'Agriculture ou celles concernant l'adaptation de l'agriculture en faveur du changement climatique ;

- le succès des RITA dans les Dom, débouchant sur une deuxième phase du programme ;

- le lancement de la plateforme partenariale de recherche agronomique pour l'océan Indien (PReRAd) ;

- et de beaux résultats engrangés par notre recherche !

2014... Une année productive et utile pour le Cirad.

Merci à toutes et à tous, personnels, partenaires, associés ou ministères, qui par votre soutien, vos actions, l'adhésion sans faille à nos valeurs, avez permis qu'il en soit ainsi ! 


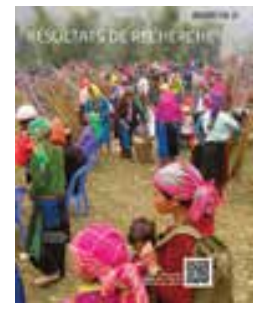

\section{RÉSULTATS DE RECHERCHE Pages 25 À 53}

\section{AGRICULTURE ECOLOGIQUEMENT INTENSIVE}

- Transition vers l'intensification écologique. Le rôle de la recherche scientifique. Interview de François-Xavier Côte ............ 26

Déchets organiques. Une mine pour l'agriculture ? ............. 26

Caféier. Des collections de reference pour mieux exploiter la diversité . 27 Palmier à huile. Un pas vers la comprehension

de l'anomalie florale mantled .......................... 27

Recherche participative. Entre savoirs paysans et savoirs scientifiques 28

Sélection du riz. Identification du gène de stérilite mâle MS-IR36 . . 28

D RP-PCP. Développer l'agriculture durable et préserver la faune

sauvage. Interview d'Eddie Mwenje . . . . . . . . . . . . . . . . . . 29

Pêche à Sumatra. Comment s'adapter au déclin des ressources ....... 30

Agrosystème Pterocarpus officinalis/taro. Symbiotes et transfert

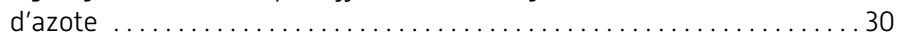

Coton cultivé. Retour aux racines . . . . . . . . . . . . . . . . . . . 30

Des génomes séquencés : tilapia, caféier, agrumes ............... 31

Production du caoutchouc naturel. L'éthylène, une hormone

essentielle ........................................ 31

Climat et pertes variétales. De mémoire d'agriculteurs ........... 32

Canne à sucre. Estimer les rendements des petits producteurs

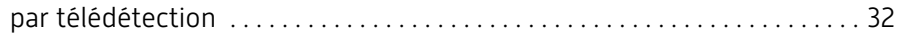

D Asap. Agrosystèmes productifs et écologiques en Afrique de l'Ouest. Interview de Souleymane Ouedraogo.

\section{VALORISATION DE LA BIOMASSE}

Durabilité naturelle du teck. Une technique rapide de prévision . . . . . 34 Agrocarburants au Burkina Faso. Les circuits de proximité stimulent la demande ........................................ 34 Biocombustibles. Projet Catapult .............................. 34

\section{ALIMENTATION DURABLE}

Aliments-mangeurs. Moins de sens, plus de distance.

Interview de Muriel Figuié

Maraîchage urbain au Vietnam.

La qualité sanitaire passe par le conseil technique .............. 36

Riz cuit. Un modèle pour prédire sa texture ................. 36

Banane plantain. Aptitude à la cuisson et qualité nutritionnelle ...... 36

Micro-organismes et fermentation des produits alimentaires

traditionnels

\section{SANTE DES ANIMAUX ET DES PLANTES}

Lutte intégrée contre les insectes. " Il est indispensable

de considérer 'ensemble du système ».

Interview de Jérémy Bouyer et Thierry Brévault ........... 38

Varioles et peste des petits ruminants. Un vaccin bivalent à l'épreuve de l'immunité acquise .................................... 39

Les florendovirus. Un nouveau genre viral des plantes à fleurs ..... 40

Avec ou sans plantes de couverture.

Qui mange le charançon du bananier?
Résultats majeurs. Deux génomes de trypanosome séquencés ...... 40 Conflit ou synergie ? Combattre l'érosion et réguler les bioagresseurs du mil .................................... 41 Cultures fruitières a la Réunion. De nouveaux pièges pour les mouches

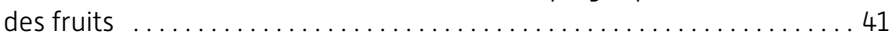

Faune sauvage, biodiversité et santé, quels défis ? ............. 41

Echaudure des feuilles de la canne à sucre. La variété a un rôle déterminant dans la contamination + Un nouveau mécanisme d'infection découvert ................................ 42

Riz pluvial à Madagascar. L'agriculture de conservation

pour limiter la pyriculariose ........................ 42

Igname africaine. Des séquences de badnavirus détectées

dans son génome ................................... 43

Lancement du réseau PACSUN. Le manioc sous surveillance . . . . . . . . 43

Chancre asiatique des agrumes. Mieux connaître

la bactérie responsable ............................... 44

Maladies animales et zoonotiques.

L'Europe et la Chine lancent Linktads ............................ . 44

Maladies infectieuses. "La répartition des chauves-souris africaines influe sur leur diversité virale».

Interview de Mathieu Bourgarel

\section{ACTION PUBLIQUE POUR LE DEVELOPPEMENT}

Paiements pour services environnementaux (PSE].

Restaurer la forêt en réduisant les inégalités.

Interview de Damien Jourdain . 46

PSE. Des instruments de marchandisation de la nature ? . . . . . . . . 47

Projet PESMIX. Atelier international à Montpellier .............. . 47

Gestion des ressources au Sénégal. Des jeux pour concevoir

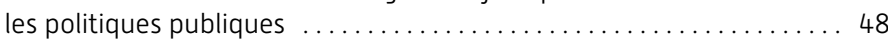

Investissements agro-industriels. Risques et opportunités

pour les pays d'Afrique centrale ........................ 48

Coopération pour le développement. Un secteur en pleine mutation . 48

D Amazonie. Promouvoir des formes durables de développement en Amazonie. Interview de Joice Ferreira ............ 49

\section{SOCIETES, NATURE ET TERRITOIRES}

Au-delà de la vulnérabilité. La résilience

des socio-écosystèmes. Interview d'Aurélie Botta . . . . . . . . 50

Forêts aménagées. Un réseau mondial pour mesurer leur résilience .. . 51 Costa Rica. Biodiversité et services ecosystémiques font bon ménage . 51 Forêts tropicales humides. Le changement climatique pourrait entraîner une baisse de la productivité ...................... 51 Surexploitation des aquifères au Maroc. Accompagner les acteurs

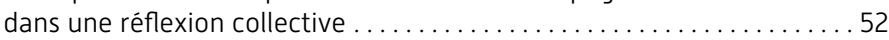
Gestion territoriale de l'environnement. Placer les acteurs au centre . . 52 I. Sirma. Partenariat scientifique multi-institutionnel et agricultures irriguées au Maghreb. Interview d' Ali Hammani ............ 53

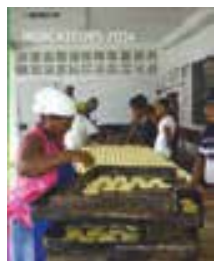

INDICATEURS 2014

Pages 54 à 60

ORGANISATION GÉNÉRALE, ADRESSES Pages 61 à 68 42 . . . . 44

(1)




\section{Le Cirad est l'organisme français de recherche agronomique et de coopération internationale pour le développement durable des régions tropicales et méditerranéennes.}

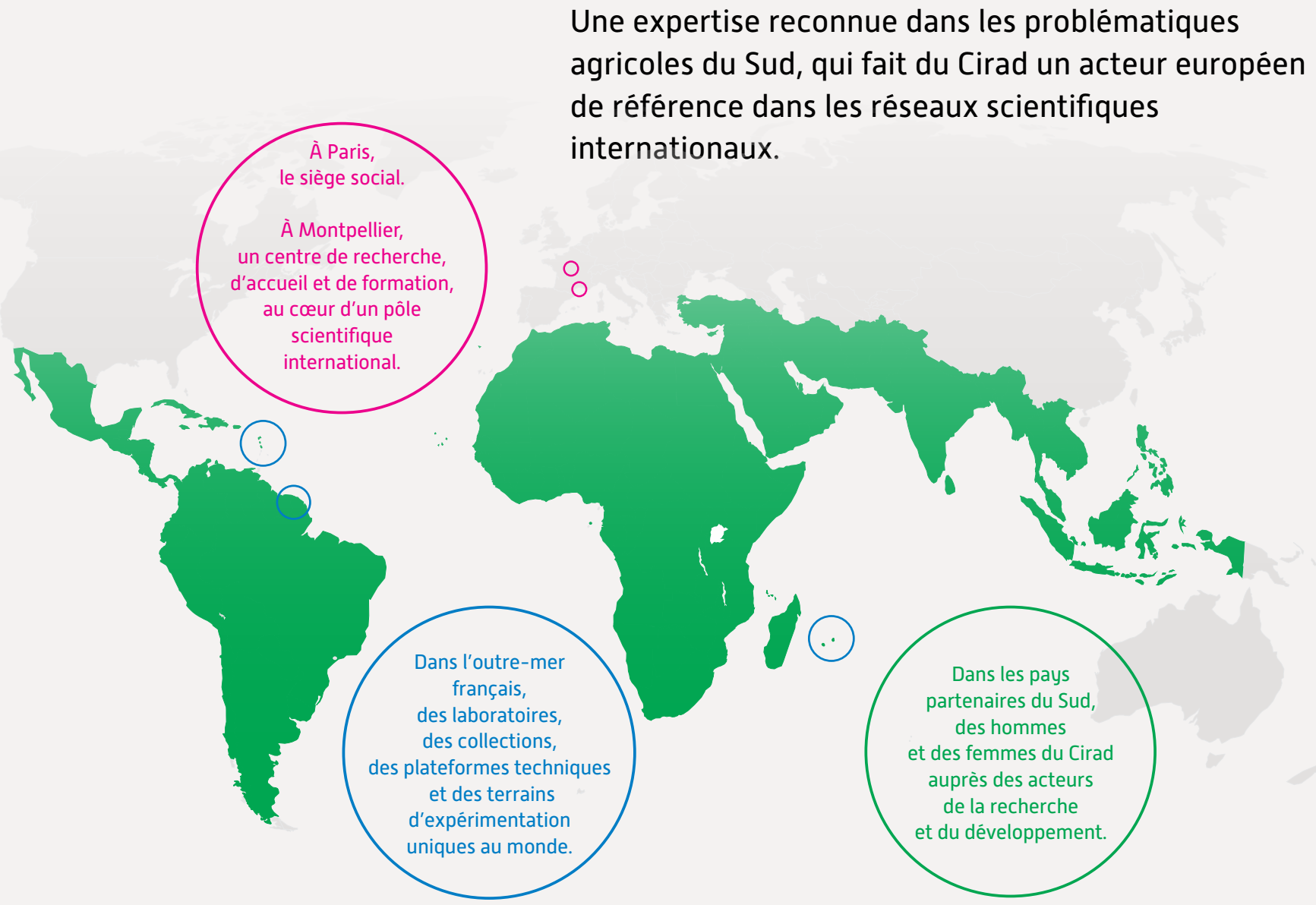

Une programmation scientifique

qui répond aux besoins des populations

locales et aux grands défis

de l'agriculture mondiale.
Organisme de recherche finalisée, le Cirad conçoit le partenariat comme une composante fondamentale de sa stratégie scientifique.

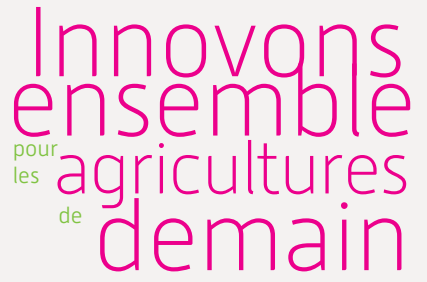

$>$ Les femmes et les hommes du Cirad imaginent des solutions originales et adaptées aux besoins des sociétés rurales et à leur environnement.
$>$ Le partenariat est la modalité et la finalité de leur action.
$>$ Ces femmes et ces hommes s'engagent pour construire une agriculture durable et capable de nourrir 9 milliards d'êtres humains en 2050. 


\section{Cirad \\ 1650 \\ U) $\bigcup$ agents \\ $200 \mathrm{M} €$}

de budget en 2014

1 er opérateur de recherche agronomique

dans l'outre-mer français

\section{Enseignement}

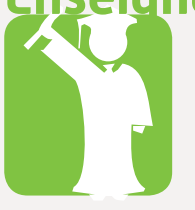

5400 heures de cours par an

300 doctorants encadrés chaque année, dont 200 originaires des pays du Sud

Partenaire de 120 cursus d'enseignement supérieur (master à école doctorale), en France et à l'étranger

\section{Partenariat}

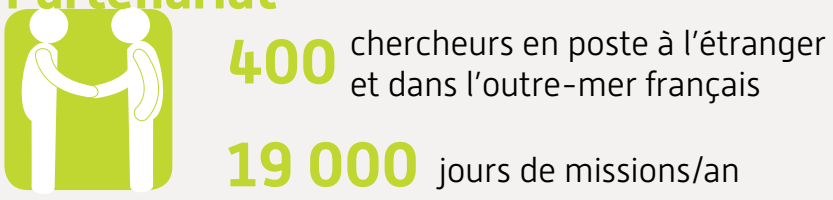

Des activités dans plus de 100 pays en partenariat avec 150 institutions

\section{Recherche}

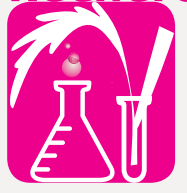

800 articles scientifiques publiés chaque année, dont 400 avec les chercheurs de pays du Sud

Plus de $\mathbf{2 0}$ dispositifs de recherche et d'enseignement en partenariat dans le monde

800 chercheurs et techniciens du monde entier accueillis chaque année
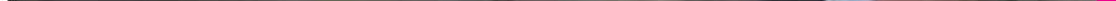


\section{EVOLUER POUR ASSUMER NOS AMBITIONS}

\section{Développement durable Un engagement de l'entreprise}

Le Cirad, établissement de recherche finalisée, a pour mission de contribuer à la viabilité à long terme des sociétés et des écosystèmes, avec une attention particulière à la situation des populations les plus démunies. II s'applique à être exemplaire dans ses propres pratiques et leurs impacts... Aussi a-t-il plus fortement affirmé son engagement dans cette voie en signant en septembre, au-delà des exigences légales, la Charte du développement durable des établissements publics et entreprises publiques.

Par cette adhésion, le Cirad partage les valeurs et les principes d'action du développement durable avec 61 établissements. II les traduit par des approches de qualité et d'amé- lioration continue dans ses systèmes de management et ses activités. Il en prend la responsabilité, portée par la direction générale, mais aussi développée par l'ensemble de ses agents. Il en rendra compte régulièrement au Conseil d'administration et proposera un plan stratégique fixant des priorités en fonction des moyens disponibles.

leandre.mas@cirad.fr

Délégation Qualité et développement durable notamment aux Rencontres de partage avec les pairs, élaborées et réalisées dans le cadre du Club développement durable des établissements publics et entreprises publiques.

\section{LE CIRAD DÉVELOPPE DÉJÀ UNE APPROCHE SOCIALEMENT VI ET ÉCOLOGIOUEMENT RESPONSABLE DE SES ACTIVITÉS}

La gestion responsable ou " l'éco-responsabilité " des activités est déclinée dans les procédures de diverses actions : amélioration énergétique ; gestion des déchets ; achats, équipement et consommation. Elle s'appuie sur des référentiels reconnus comme la démarche qualité et le management environnemental [ 3 bilans carbone réalisés].

Deux sites [Montpellier et Martinique) ont déjà développé un plan d'action environnement et un dispositif de suivi, en particulier pour la réduction d'émission de gaz à effet de serre. Les 6 sites du Cirad devraient en être pourvus d'ici 2017.
La gestion des ressources humaines vise à respecter les enjeux de la diversité (parité hommes-femmes, gestion du handicap, seniors...), à prévenir les risques psychosociaux et à développer les compétences de chacun.

Des relations constructives sont développées avec les collectivités locales (métropole, outre-mer]. Notamment, les connaissances et les savoirs produits par le Cirad sont mis au service des citoyens et des décideurs pour les aider à prendre des décisions conscientes et responsables : recyclage des déchets à la Réunion, environnement et gestion de la chlordécone aux Antilles, valorisation et conservation de la biodiversité et des écosystèmes fragiles..

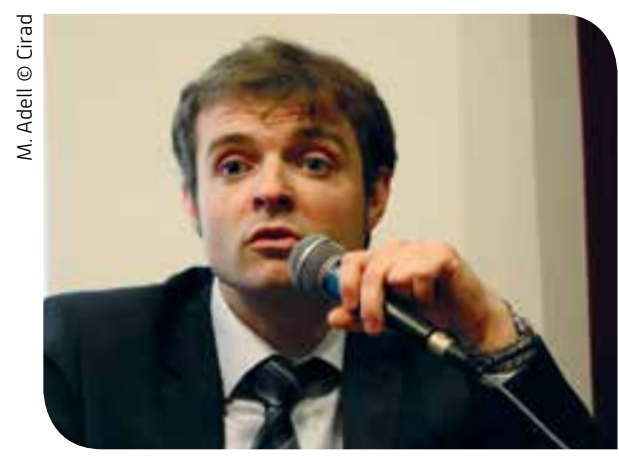

\section{François Pouget, nouveau directeur délégué aux ressources}

François Pouget, économiste de trente-trois ans, est le nouveau directeur général délégué aux ressources et aux dispositifs du Cirad depuis le 2 juin. Il était précédemment en poste au ministère des Finances et des Comptes publics. Il représentait le ministre chargé du budget au Conseil d'administration du Cirad.

La direction aux ressources et aux dispositifs regroupe toutes les fonctions support du
Cirad. François Pouget a pour principale mission la mise en œuvre de la stratégie de l'établissement et de son contrat d'objectifs dans le champ de l'appui à la recherche. II a en charge les relations sociales, la présidence du comité d'entreprise et un important chantier mis en place afin de développer les ressources propres du Cirad : le Pacte de développement des ressources. 


\section{Un nouveau contrat d'objectifs signé avec les tutelles}

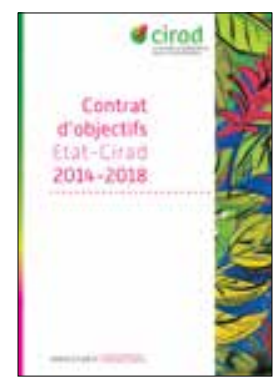

Le contrat d'objectifs 2014-2018 a été signé entre le Cirad et ses tutelles. II décline en objectifs prioritaires, partagés avec l'Etat, les quatre ambitions avec lesquelles notre organisme a revisité sa stratégie pour les dix prochaines années.

Ce contrat engage le Cirad à participer activement à la stratégie nationale de recherche et à développer ses partenariats au Sud dans le cadre d'une coordination de la recherche agronomique internationale. II donne aussi la priorité à un certain nombre de chantiers de modernisation du fonctionnement de l'établissement. L'objectif est de consolider son modèle économique en renouvelant son portefeuille contractuel en cohérence avec sa stratégie scientifique et partenariale. Le Cirad est bien placé pour faire valoir ses avantages comparatifs, liés à sa capacité reconnue de coopération entre pays du Nord et pays du Sud. Michel Eddi, PDG du Cirad, le ministre Laurent Fabius (Affaires étrangères et Développement international), les secrétaires d'État Annick Girardin (Développement et Francophonie) et Geneviève Fioraso [Enseignement supérieur et Recherche] y ont apposé leur signature.
Faire référence au niveau mondial sur nos priorités scientifiques.

Co-construire des partenariats stratégiques de recherche agronomique pour le développement.

Développer les conditions d'une innovation efficace.

Evoluer pour assumer nos ambitions.

\section{A propos des dispositifs de recherche et d'enseignement en partenariat [DP]}
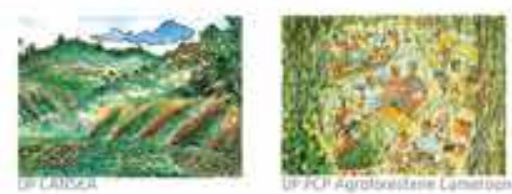
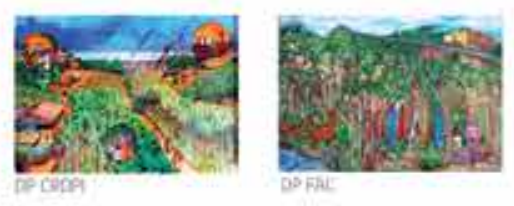
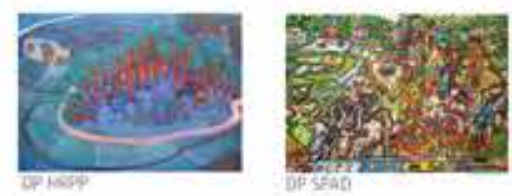

Les dispositifs de recherche et d'enseignement en partenariat [DP] sont la forme la plus aboutie, mais non exclusive, que le Cirad a élaborée pour construire des partenariats à long terme avec les institutions du Sud sur des thématiques partagées.

Pour promouvoir les 21 DP auprès des bailleurs, le Cirad a réalisé un ensemble de fiches qui met en avant l'expertise, les atouts et les réseaux dans lesquels ils sont impliqués. La réalisation de tableaux par des artistes-peintres locaux, dont la mission était d'évoquer leur thématique, marque leur enracinement dans une identité scientifique et culturelle. Ces œuvres sont exposées dans le hall du centre de recherche de Montpellier.

Dans le cadre de ses DP, le Cirad privilégie un partenariat contractuel de proximité [156 organismes partenaires en 2014], mais aussi entre dispositifs. Ce partenariat est ouvert à tous les acteurs de l'innovation dans les domaines de l'agriculture, de l'alimentation et de l'environnement. L'objectif à terme est de faire reconnaître les DP comme plateformes partenariales dans les pays du Sud, au même titre que les autres dispositifs français, notamment via l'Alliance nationale de recherche pour l'environnement, Allenvi.

Dans son document de stratégie à l'international, la direction d'Agreenium, dont le Cirad est membre, écrit, que « les DP doivent être considérés par tous les membres d'Agreenium comme des lieux prioritaires d'investissement » dans les pays en développement.

jacques.pages@cirad.fr

Délégué aux dispositifs de recherche et d'enseignement en partenariat
Tableau réalisé pour le DP Malica (c) Tran Thanh Hang

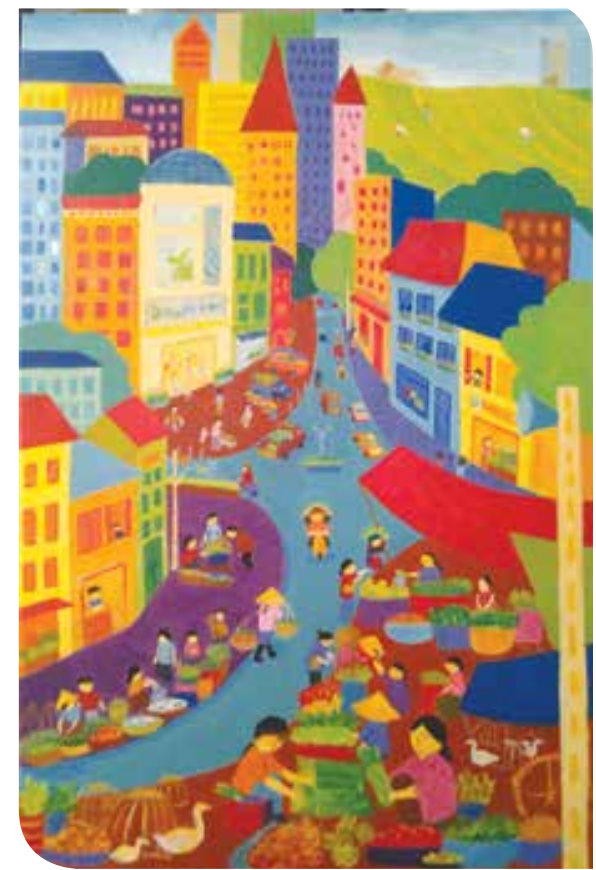




\section{Une programmation à 5 ans des unités de recherche}

Toutes les unités de recherche (UR) du Cirad ont été évaluées par l'Agence d'évaluation de la recherche et de l'enseignement supérieur (AERES). La construction d'une trajectoire scientifique contribuant à la réalisation des chantiers prévus au titre des "Objectifs de stratégie scientifique et partenariale » (OSSP) est inscrite dans le contrat d'objectifs 20142018. Cette construction mobilise trois modalités de pilotage : le pilotage "par conviction», fondé sur le débat interne, qui favorise l'ap- propriation des priorités stratégiques; la démarche "incitative », par l'allocation de moyens financiers ciblés; la " contractualisation sur objectifs", au travers de "Lettres annuelles d'objectifs » (LAO) dans le cadre du budget annuel de l'établissement, pour les UR comme pour les services d'appui.

Les projets scientifiques des unités de recherche et leur mise en œuvre vont également être contractualisés pour une durée de 5 ans sous la forme de "Lettre pluriannuelle d'objectifs » (LPO), complétant ainsi le dispositif établi avec les LAO.

La LPO met en cohérence les stratégies des unités et de l'établissement, en particulier pour les trois types principaux de déclinaisons les axes stratégiques, les DEUX CHANTIERS THÉMATIQUES de l'établissement et les dispositifs de recherche et d'enseignement en partenariat (DP).

\section{DEUX CHANTIERS THÉMATIQUES}

\section{Développer les conditions d'une innovation efficace}

\section{INNOVATION ET IMPACT}

Comment et par quels canaux les résultats de la recherche génèrent-ils ou contribuent-ils, par I'innovation, à l'amélioration du développement des sociétés rurales du Sud ? Comment qualifier et quantifier cet impact dans la stratégie de l'établissement ? Pour sa première année, le groupe de travail chargé de ce chantier a sélectionné treize cas représentant la diversité des actions du Cirad. II a organisé un atelier en octobre avec des chercheurs porteurs de ces études de cas et des experts extérieurs pour valider et améliorer le cadre méthodologique de son expertise.

L'objectif à cinq ans du chantier « Innovation et impact » est de mettre au point une méthodologie et une pratique d'évaluation de l'impact des recherches du Cirad, tant en interne que pour les tutelles et les bailleurs, en s'appuyant sur des études de cas. Le chantier comporte quatre volets : monter une plateforme méthodologique ; explorer la diversité des innovations et de leurs chemins d'impact ; développer une capacité de recherche sur les liens entre innovation et impact ; organiser la diffusion d'une " culture de l'impact ».

etienne.hainzelin@cirad.fr danielle.barret@cirad.fr

Coordinateurs du chantier « Innovation et impact »
Hypothenemus hampei femelle, scolyte du caféier. (๑) Cirad

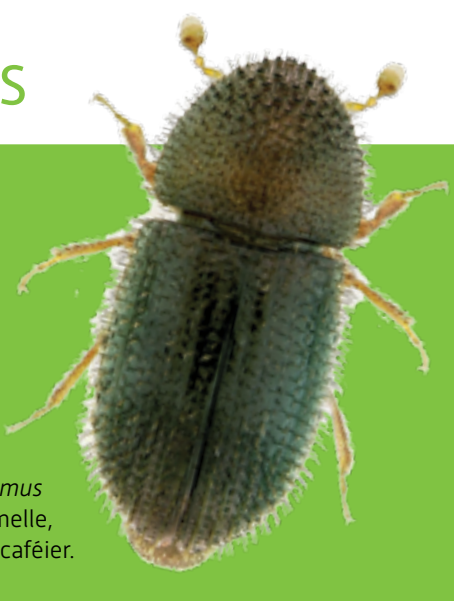

Promouvoir l'ambition de l'intégration scientifique au service du développement durable

\section{FILIÈRES ET DÉVELOPPEMENT DURABLE}

Comment assurer impératifs de production et du développement durable ? Dans de nombreux pays tropicaux, un nombre réduit de filières agricoles joue un rôle économique et social majeur. Or l'augmentation de la production mondiale fait craindre un épuisement des ressources et des déséquilibres planétaires majeurs. Dans cette perspective, les approches techniques et biologiques des systèmes agroalimentaires doivent être profondément renouvelées.

Le Cirad dispose de solides atouts sur les filières tropicales, avec de nombreux savoir-faire, résultats et bases de données remarquables, une expertise mondialement reconnue et des liens consolidés dans le temps avec divers partenaires des filières, privés et publics. Sa compréhension approfondie des modèles biologiques, des systèmes d'exploitation et des formes d'organisation des filières dans de nombreux pays lui confère une crédibilité auprès des partenaires et une pertinence scientifique et sociale. Les démarches engagées notamment sur le palmier à huile et le bananier sont prometteuses et riches d'enseignements pour promouvoir des approches renouvelées.

Le chantier Filières et développement durable, qui commencera en 2016, sera organisé selon 4 volets : animation scientifique et partage de méthodologies ; production scientifique intégrée ; expertises, prospective ; aide à la décision pour l'engagement auprès d'opérateurs privés.

estelle.bienabe@cirad.fr Adjointe au DGD-RS

hubert.omont@cirad.fr Délégué aux filières

Pépinière industrielle, Congo. ๑ D. Louppe/Cirad

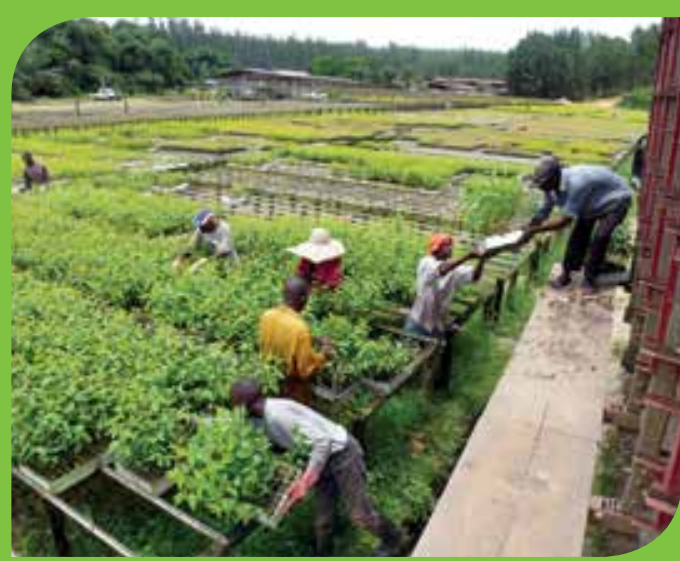




\section{Patrimoine numérique 60 ans de recherche à valoriser}

A l'ère du tout numérique, les nouvelles formes de collecte de données scientifiques (plateformes partagées, recherche participative...), d'analyse (fouilles et science des données) et de diffusion de la connaissance (données associées aux publications) ouvrent la voie à l'open science. En outre, la prise en compte des grands enjeux de la planète (changement climatique, pression sur les écosystèmes...) entraîne un regain d'intérêt de la recherche pour des données anciennes qui pourraient être remobilisées et associées aux nouvelles pour étudier certaines dynamiques. Cela pose la question du partage des données au sein de communautés variées d'utilisateurs.

Conscient de ces enjeux, le Cirad a amorcé un travail de veille externe et de réflexion avec ses partenaires des UMR (Inra, IRD...). Une initiative a été lancée autour du patrimoine numérique du Cirad, ciblant principalement les données scientifiques. Un groupe de travail a ainsi été créé pour produire un état de l'art et des pratiques de l'organisme. Il doit également proposer un plan d'action et formuler des recommandations. Tous les niveaux d'organisation sont impliqués (départements et UR pilotes, services d'appui, ressources humaines, éthique et déontologie...).

Certaines données issues de plus de 60 années de recherche dans les régions tropicales pourraient ainsi être revisitées. Quant aux données acquises sur les nouveaux projets, elles bénéficieront de nouveaux plans de gestion en vue de leur réutilisation.

pascal.bonnet@cirad.fr

Département Environnements et sociétés [ES]

Mise en place de boutures de manioc à Madagascar. (c) Cirad. Support : plaque de verre

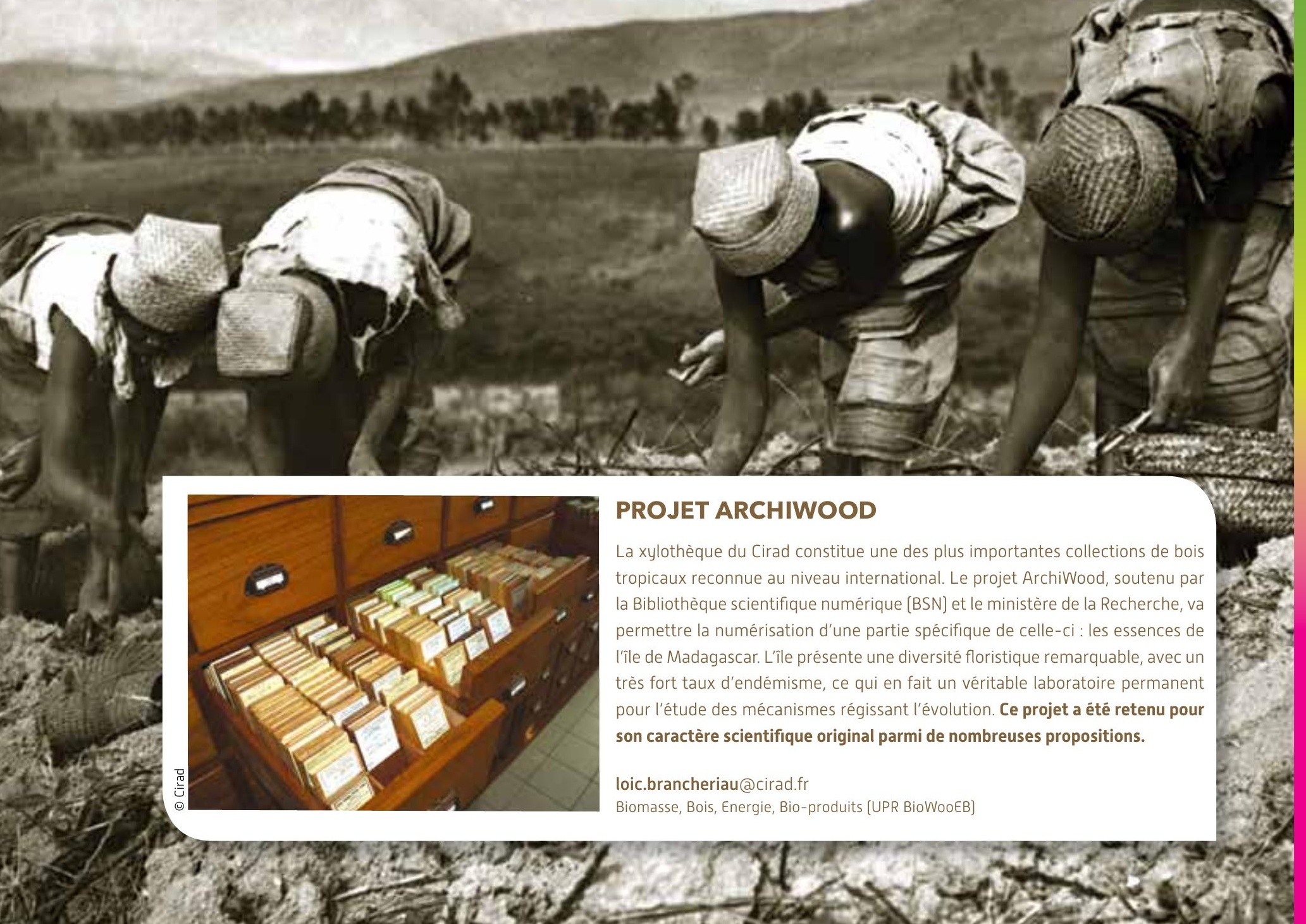




\section{PRODUIRE ET RENFORCER DES PARTENARIATS STRATÉGIQUES}

\section{Sécurité alimentaire Métaprogramme Cirad-Inra \\ Comment nourrir durablement, sainement et équitablement dix milliards d'humains à l'horizon 2050 ?}

Face à ce défi, le Cirad, l'Inra et leurs partenaires français ont décidé de revisiter cette question et de construire une programmation commune. Le métaprogramme « GloFoods, Transitions pour la sécurité alimentaire mondiale », a ainsi tenu son séminaire de lancement à Montpellier en juin avec 140 participants de l'enseignement et de la recherche. En juillet, certains d'entre eux ont continué leurs réflexions lors de la réunion internationale sur la gouvernance de la sécurité alimentaire organisée par la FAO et I'Iddri. Un appel à manifestation d'intérêt a été lancé en décembre. Il permettra d'attribuer des fonds incitatifs aux équipes porteuses de projets innovants.

etienne.hainzelin@cirad.fr

co-directeur de Glofoods

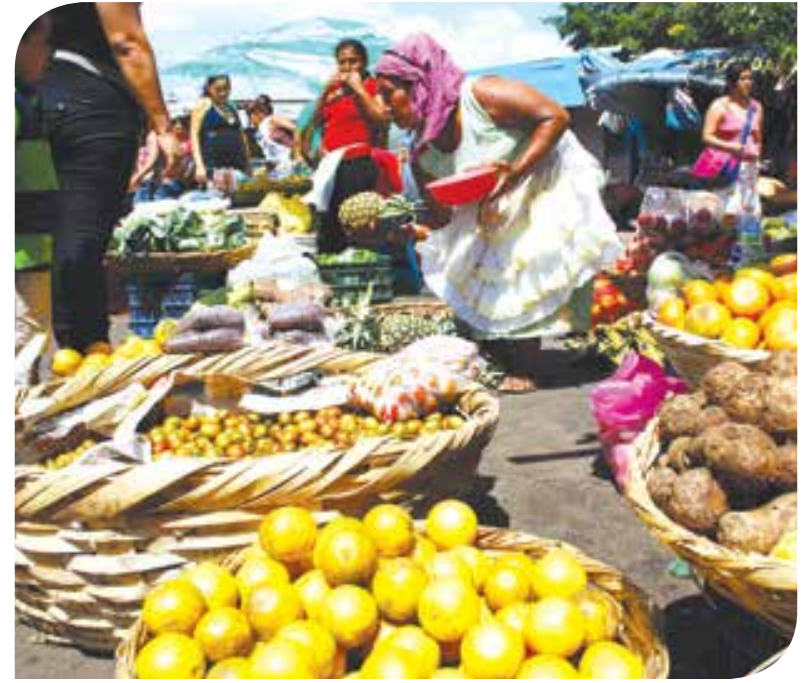

Marché au Nicaragua. S. Fréguin @ Cirad

\section{Europe}

\section{Nouvelles initiatives pour l'Afrique}

Le Cirad, acteur du dialogue EuropeAfrique en recherche et innovation. A la suite du $4^{\mathrm{e}}$ sommet Union européenneAfrique à Bruxelles en avril, un groupe de 10 experts a été chargé par la Commission européenne et la Commission de l'Union africaine d'élaborer une feuille de route pour un partenariat dans les secteurs de la sécurité alimentaire et nutritionnelle et de l'agriculture durable. Le Cirad, représenté par Philippe Petithuguenin, co-préside ce groupe, qui s'inscrit dans le cadre du dialogue de haut niveau (HLPD) entre I'UE et I'Afrique sur les sciences, les technologies et l'innovation. La feuille de route fait l'objet d'une large consultation. Elle propose de développer le partenariat UEAfrique autour de trois piliers thématiques: intensification durable; systèmes agricoles et alimentaires pour la nutrition; expansion et amélioration des marchés agricoles et du commerce, et un pilier transversal : ajouter de la valeur aux coopérations existantes; faciliter les processus d'innovation; renforcer les capacités de recherche et d'innovation; mettre en place une gouvernance adaptative]. Elle devrait être adoptée en 2015.

L'initiative "IntensAfrica " soutenue dans le cadre du programme « Horizon 2020 ». «ProlntensAfrica » a été acceptée par la Commission européenne en novembre. C'est une proposition originale qui vise à valider en deux ans la faisabilité d'une programmation de recherche conjointe à grande échelle entre l'Europe et I'Afrique. Elle permettra de faire progresser l'initiative IntensAfrica, portée par le Cirad et I'université de Wageningen depuis 2013. Cette initiative rassemble les partenaires scientifiques de 13 pays européens et de nombreux pays africains du Forum for Agricultural Research in Africa (Fara) autour d'une ambition commune : I'intensification durable des systèmes de production agroalimentaires. jean-michel.sers@cirad.fr Délégué Europe
Nouveau rendez-vous annuel avec les décideurs. Le Cirad lance "See you in Brussels! », un cycle de conférences annuelles à Bruxelles. Son objectif est de dialoguer régulièrement avec les décideurs en tant que partenaire majeur des politiques de recherche, de coopération et de soutien aux régions. La première édition s'est tenue le mardi 2 décembre dans les locaux de la Maison de la Région Languedoc-Roussillon, en présence de Michel Eddi, Patrick Caron et Philippe Petithuguenin. Jean-Michel Sourisseau et Pierre-Marie Bosc, pour le Cirad, et José Antonio Osaba, pour le World Rural Forum, ont présenté un bilan de l'Année internationale de l'agriculture familiale, et notamment les travaux du Cirad, à une cinquantaine de personnalités et décideurs très intéressés de la Commission et du Parlement européens, du Secrétariat Afrique-Caraïbes-Pacifique et des ambassadeurs des pays du Sud auprès de I'UE.

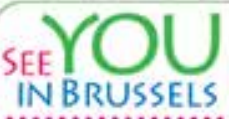
INBRUSSELS 


\section{Le Cirad rejoint l'Alliance globale pour l'agriculture climato-intelligente}

A quinze mois de la Conférence des parties sur le changement climatique de Paris en décembre 2015 (Cop 21), le secrétaire général des Nations unies, Ban Ki-Moon, a convoqué un sommet pour le climat, le 23 septembre à New York. L'Alliance globale pour l'agriculture climatointelligente a été lancée à cette occasion. Elle a pour objectif d'accroître la productivité agricole et les moyens de subsistance de 500 millions d'agriculteurs du monde entier, de renforcer la résilience des systèmes alimentaires et de réduire les émissions de gaz à effet de serre liées à l'agriculture. L'Alliance est organisée autour de trois piliers : renforcement des connaissances et partage d'information, mobilisation de financements publics et privés et renforcement de leur efficacité, et création d'un environnement politique favorable.

La charte a été signée, pour la France, par Annick Girardin, Secrétaire d'Etat au Développement et à la Francophonie, qui a rappelé que l'Etat français s'engage à promouvoir une agriculture climato-intelligente fondée sur une approche agroécologique et s'appuyant sur le modèle de l'agriculture familiale tant au plan national qu'à l'international. Michel Eddi a également signé l'adhésion du Cirad à cette charte selon les lignes présentées par Annick Girardin.

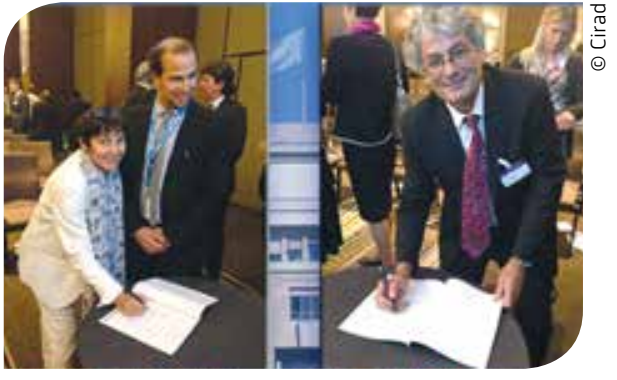

Annick Girardin (à gauche) et Michel Eddi (à droite)

emmanuel.torquebiau@cirad.fr chargé de mission changement climatique

\section{Symposium France-FAO sur l'agroécologie}

Le symposium international sur l'agroécologie pour la sécurité alimentaire et la nutrition dans le monde, qui s'est tenu en septembre à Rome (Italie), est une initiative conjointe de la FAO et du ministère français de l'Agriculture. Le Cirad était co-organisateur et sponsor de ce symposium aux côtés de la FAO et de l'Inra. L'objectif de ces journées était de promouvoir un dialogue rassemblant les expériences et les politiques existantes sur l'agroécologie. Leur originalité était de faire se rencontrer scientifiques, ONG, acteurs privés et publics. Le symposium a accueilli 400 participants de 61 pays et suscité l'intérêt de plus 60 médias du monde entier. Le Cirad a été très actif dans l'animation scientifique avec trois conférences introductives de sessions, des animations de tables rondes et de nombreux posters.

Une délégation de huit personnes accompagnait Stéphane le Foll, ministre de l'Agriculture, de l'Agroalimentaire et de la Forêt, qui
Stéphane Le Foll, ministre de l'Agriculture, de l'Agroalimentaire et de la Forêt à la tribune.

a notamment promu «le projet agroécologique » français. Le ministre a aussi soutenu les 3 rencontres régionales qui ont été proposées pour la suite, avec la possibilité d'accueillir l'une d'elles à Paris en 2016. La France a également proposé d'appuyer la création d'un «Club des pays amis de l'agroécologie » et les échanges d'informations scientifiques et de pratiques localement adaptées.

Après la conférence, Jacques Pagès, représentant de la France à la $\mathrm{FAO}$, a réuni les scientifiques du Cirad présents et Barbara Herren, présidente du comité scientifique du colloque, pour envisager des actions concrètes sur le terrain associant la FAO et certains dispositifs

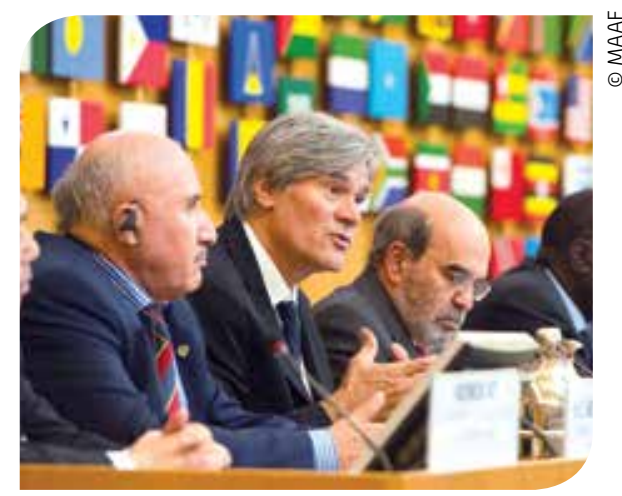

de recherche et d'enseignement en partenariat (DP) du Cirad. Parmi les collaborations envisagées, le renforcement des connaissances scientifiques des représentants de la FAO et l'organisation de Farmers' Fields Schools ont été évoqués.

etienne.hainzelin@cirad.fr Conseiller du PDC

Rapport final du colloque et interventions : http://www.fao.org/about/meetings/afns/fr

Le projet agro-écologique en France : http://agriculture.gouv.fr/agroecologie-une-force-pour-la-france 


\section{Collaborations renforcées avec les pays ACP}

Le premier accord-cadre formel entre le Cirad et le secrétariat des pays d'Afrique, des Caraïbes et du Pacifique (ACP) a été signé le 9 décembre à Bruxelles. Le Cirad travaille déjà très étroitement avec de nombreuses institutions des pays ACP en bilatéral. Le 8 avril, le centre de Montpellier avait accueilli plusieurs ambassadeurs et représentants des pays ACP producteurs de sucre. A l'occasion de cette visite, Michel Eddi et Patrick Gomes, président du sous-comité des pays ACP et ambassadeur de Guyana, avaient renouvelé leur vœux de collaboration scientifique et technique sur des problématiques agricoles telles que les bioénergies, les biomatériaux, les approches sociales de la production ou encore l'intensification écologique. La signature a eu lieu lors de la $100^{\circ}$ réunion du Conseil des ministres ACP à Bruxelles. Philippe Petithuguenin, directeur adjoint à la recherche et à la stratégie, y représentait le Cirad.

philippe.petithuguenin@cirad.fr

Direction de la recherche et de la stratégie

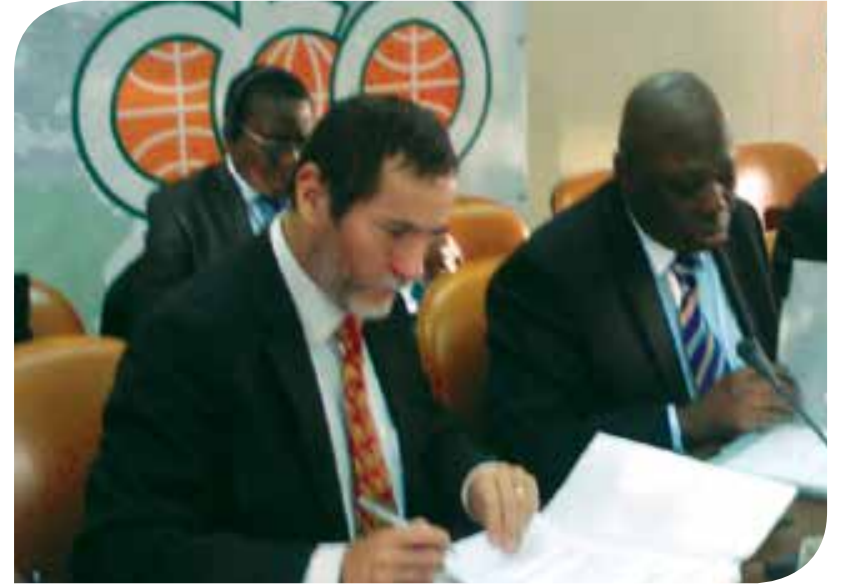

Philippe Petithuguenin et Alhaji Muhammad Mumuni, secrétaire général de l'organisation des pays ACP, à Bruxelles. ๑ J.-C. Dagallier/Cirad

\section{Succès des réseaux d'innovation et de transfert agricole (RITA)}

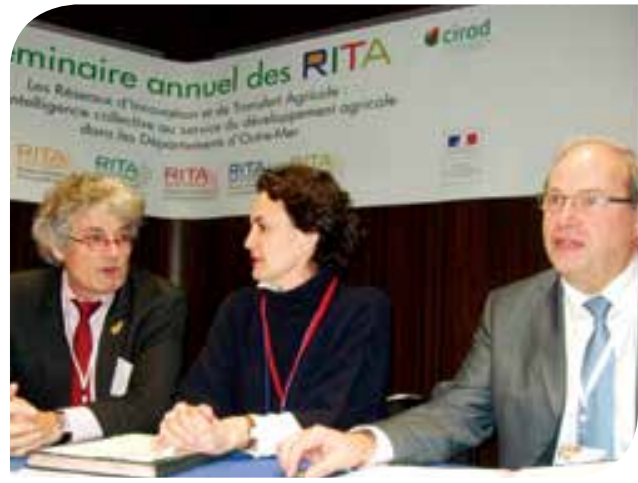

Renouvellement de la Convention entre le ministère de l'Agriculture, le Cirad et l'Acta. @ S. Della Mussia/Cirad
Evaluation et certification de nouvelles variétés végétales (ignames, bananes, ananas, agrumes, abricots pays, plantes aromatiques et médicinales...), mise en place de nouveaux systèmes de cultures fruitières et maraîchères à bas niveau d'intrants chimiques, amélioration de l'élevage bovin, porcin et cunicole, et aussi meilleure connaissance de l'abeille et de ses

La première phase de mise en place des Réseaux d'innovation et de transfert agricole (RITA 1) s'est achevée fin 2014. Des bilans ont été dressés à l'occasion de divers événements nationaux comme le séminaire organisé par l'Acta, le Cirad et l'Odeadom au Salon international de l'agriculture à Paris, et lors d'événements régionaux, comme le séminaire du 9 décembre en Martinique.

Des bilans par projet ont également été dressés comme celui de Eva-transfert, en Guadeloupe le 25 novembre. Tous soulignent l'efficacité de ce dispositif multipartenarial animé par l'Acta et le Cirad et mis en place fin 2011 par le ministère de l'Agriculture afin de diversifier et promouvoir les productions animales et végétales dans l'outre-mer français.

produits... Que ce soit à la Réunion, à Mayotte, à la Guadeloupe, à la Martinique ou en Guyane, en deux années, les projets de la première vague (RITA 1) recueillent déjà des résultats concrets.

Le fonctionnement en réseau a permis d'impliquer tous les acteurs, de la recherche au développement agricole, en passant par la formation. Des innovations ont été développées avec les producteurs pour améliorer la durabilité économique et environnementale des productions. Les RITA sont ainsi cités en exemple en tant que dispositif multipartenarial efficace. Ils figurent en bonne place pour bénéficier des nouveaux programmes de développement européens, nationaux et régionaux.

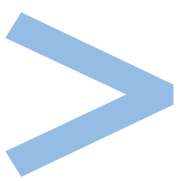




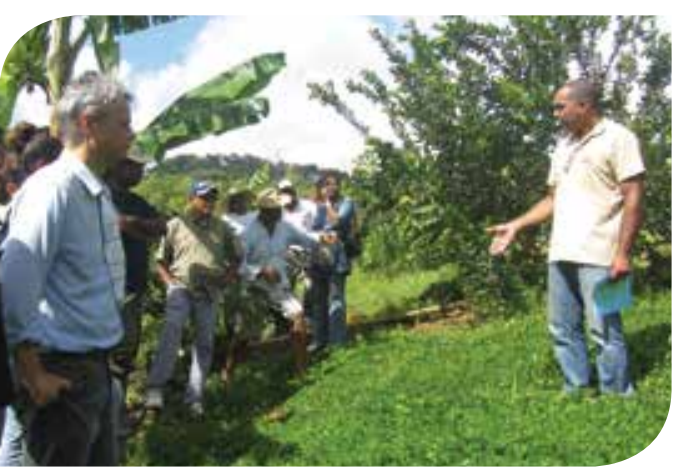

Visite de terrain à la Martinique : plantes de couverture en bananeraie. (c) Cirad

\section{... à RITA 2}

La volonté commune, avec RITA 2 (2015-2020), est de développer de nouvelles pratiques agroécologiques avec le plus grand nombre d'agriculteurs. En 2015, de nouveaux projets vont voir le jour. En Guadeloupe, le RITA intégrera les acteurs de la filière canne. En Martinique, des projets de transformation des produits ou d'agroforesterie seront inclus. La performance des élevages et l'amélioration de la qualité des produits animaux constitueront un projet phare à Mayotte. En Guyane, les cultures de tubercules et les cultures sur abattis-brûlis seront intégrées. A la Réunion, le champ d'action s'élargit aussi avec la prise en compte de la canne à sucre et du secteur animal.

Le ministère de l'Agriculture a renouvelé le mandat d'animation du Cirad et de l'Acta. Ces organismes maintiennent un fonctionnement similaire en mettant l'accent sur la capitalisation et le partage inter-Dom des informations. Le transfert et la formation vont être développés avec les Chambres d'agriculture, les techniciens de groupements et formateurs de l'enseignement agricole. Une réflexion prospective sera menée sur l'avenir des RITA pour trouver les ressources nécessaires à la poursuite des actions au bénéfice des producteurs des Dom.

jean-marc.thevenin@cirad.fr Bioagresseurs : analyse et maîtrise du risque [UPR Bioagresseurs]

\section{Coopération régionale Une plateforme multi-acteurs pour la Caraïbe}

Dans la Caraïbe, la stratégie régionale de coopération scientifique du Cirad repose sur un fort réseau de partenaires, consolidé ces dernières années selon quatre axes thématiques : systèmes de culture agroécologiques; gestion des ressources génétiques, sélection variétale et qualité des produits ; santé animale et végétale ; dynamique des forêts tropicales. Sur le plan opérationnel, il s'agit plutôt d'un grand projet intégré, structuré en sous-projets thématiques, avec des axes d'animations transversaux autour de l'agroécologie, l'agrobiodiversité, la formation et l'impact... Il sera piloté par une plateforme de coordination multi-acteurs.

La direction régionale du Cirad a présenté la nouvelle plateforme aux acteurs publics et privés, nationaux et locaux, des cinq collectivités françaises des Amériques lors de la $11^{\mathrm{e}}$ conférence de coopération régionale Antilles-Guyane. Cette conférence est le principal rendez-vous annuel des acteurs des cinq collectivités.

Echantillons de la collection de bananiers (Martinique). (๖) Cirad

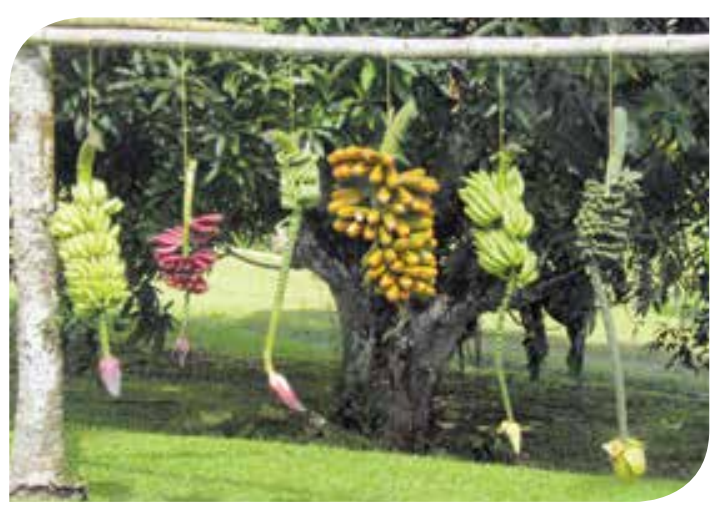

Le Centre de ressources biologiques [CRB] Plantes tropicales, aux Antilles, a été certifié selon la norme NF S96-900. C'est une reconnaissance nationale de la qualité des activités menées sur la canne à sucre, la banane, l'ananas, la mangue ou l'igname. Près de 2500 variétés sont répertoriés aux Antilles. Celles-ci sont conservées au Cirad et à l'Inra, en plein champ ou in vitro.

\section{Le PROJet BANANe durable CARAÏBE a} tenu sa réunion de clôture les 14 et 15 octobre. En quatre ans, ce projet coordonné par I'UGPBAN, I'Institut technique tropical (IT2) et le Cirad et financé par le programme européen Interreg IV Caraïbes, a appuyé le développement de systèmes de culture innovants dans toute la région. Ces approches pourraient être poursuivies dans le cadre du programme Interreg 5.

jean-michel.risede@cirad.fr

Fonctionnement écologique et gestion durable des agrosystèmes bananiers et ananas (UPR Geco)
LE PROJET DEVAG, qui s'intéressait aux filières horticoles, s'est terminé en mars. Soutenu par les Ambassades de France à Cuba et en Haïti et financé par le programme Interreg IV Caraïbes, ce projet, en avance sur toutes les directives nationales en matière d'agroécologie, est considéré comme un modèle. L'ensemble de ses produits sont disponibles en ligne et sur DVD.

paula.fernandes@cirad.fr,

Fonctionnement agroécologique et performances des systèmes de cultures horticoles [UR Hortsys)

http://devag.tropical-agroecology.org

CARIBVET, RÉSEAU CARIBÉEN de SANTÉ ANIMALE, a tenu son neuvième comité de pilotage du 5 au 6 mai à La Havane. Les chefs de services vétérinaires caribéens ont également pu assister à la conférence internationale de surveillance en santé animale (ICAHS) qui se déroulait à Cuba du 7 au 9 mai, organisée par le Centro Nacional de Sanidad Agropecuaria [CENSA) et un comité international composé de chercheurs cubains, brésiliens et européens (dont des Ciradiens). 


\section{Lancement de la plateforme de recherche agronomique pour l'océan Indien (PReRAD]}

La plateforme régionale de recherche agronomique pour le développement dans l'océan Indien, PReRAD, est lancée. Michel Eddi a signé en juin l'accord-cadre qui associe le Cirad, la Réunion et la Commission de l'océan Indien [COI], pour la création de cette plateforme. Elle sera animée par le Cirad depuis la Réunion et Mada-

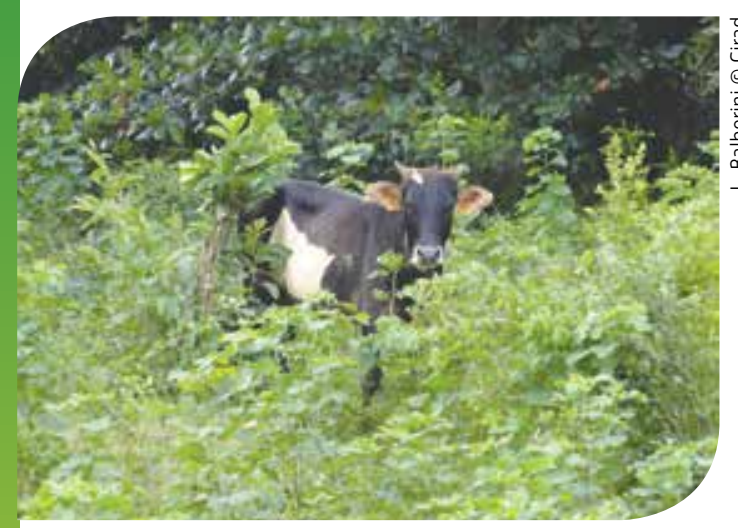

gascar et rassemble un effectif de 600 personnes, constitué pour moitié par les équipes du Cirad et pour l'autre moitié par les équipes partenaires des pays de la COI.

Cette plateforme répond à la nécessité pour les pays de l'océan Indien de renforcer leur autonomie alimentaire en développant leurs complémentarités sur des filières agricoles clés. Le déploiement d'un partenariat régional pourra bénéficier de financements internationaux complémentaires des fonds européens (Feder). Pour ce faire, la mise en place d'une gouvernance partagée avec les pays partenaires permet de structurer la coopération sur des thématiques d'intérêt commun avec les ministères, les universités et le secteur privé, , notamment: santé animale et végétale, maladies émergentes, systèmes agroalimentaires et qualité des produits, valorisation de l'agrobiodiversité et recyclage des matières organiques.
La PReRAD est le principal opérateur scientifique de la COI pour la sécurité alimentaire. Elle entend améliorer une recherche d'excellence, en appui au développement des filières agricoles. L'axe majeur est la réduction des pertes grâce à la mise en place de démarches agroécologiques. A cette fin, le Cirad renforcera ses structures d'accueil et de recherche, notamment à la Réunion, afin d'accueillir les équipes, les appuyer par des missions et les former, autant que de besoin. II bénéficiera de l'appui du consortium Agreenium pour la formation universitaire et professionnelle qui mettra en place un dispositif régional de formation à distance avec les universités.

gilles.mandret@cirad.fr Directeur régional Réunion-Océan Indien

\section{Relance de la coopération scientifique avec la Côte d'Ivoire}

Le Cirad et le Centre national de recherche agronomique (CNRA) ont signé un accord-cadre de coopération à l'occasion de la visite d'État du président de la République française en Côte d'Ivoire le 17 juillet. Cette signature symbolise la relance des activités de coopération scientifique du Cirad en Côte d'Ivoire, après la période de troubles politiques qu'a connue le pays. Elle s'est déroulée en présence du ministre ivoirien de la Recherche et de l'Enseignement supérieur.

La coopération concerne notamment la filière cacao : organisation de la filière et des producteurs, lutte contre le swollen shoot virus (CSSV) et reconnaissance de la qualité de la production ivoirienne. La banane et le plantain sont également concernés, ainsi que les cultures vivrières. La coopération sera menée selon trois axes - recherche, développement et formation - avec I'appui d'Agreenium. Elle pourra bénéficier du fonds du contrat de désendettement développement (C2D) mis en place par la France et signé par la Côte d'Ivoire en décembre 2012 dans le cadre de l'allègement de la dette des pays en développement. A cette fin, le CNRA, le Cirad et le Fonds interprofessionnel pour le conseil et la recherche agricole (Firca) de la Côte d'Ivoire construiront des propositions conjointes de programmation.

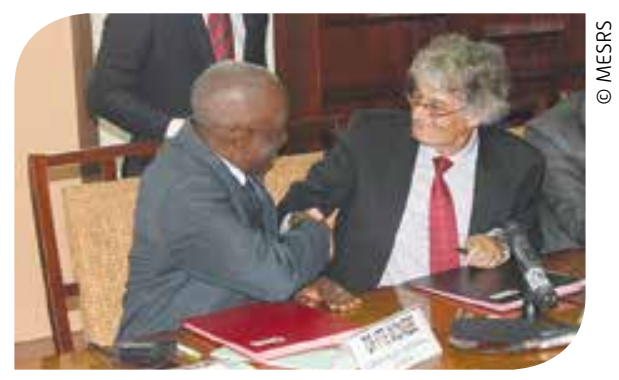

Yté Wongbé, DG du Centre national de recherche agronomique de Côte d'Ivoire et Michel Eddi.

patrice.grimaud@cirad.fr Directeur régional Afrique de l'Ouest continentale 


\section{Accord entre l'État kényan et les instituts de recherche français}

Un accord-cadre de coopération a été signé entre les trois instituts français de recherche présents au Kenya (Cirad, Ifra et IRD) et le ministère kényan de l'Education, de la Science et de la Technologie (MOEST) le 6 mai à Nairobi, sous l'égide de l'Ambassade de France.

Pour le Cirad, cet accord légitime une activité de recherche et de formation par la recherche qui, sur le territoire kényan, se concrétisait surtout par un partenariat avec les centres internationaux, l'Icraf (café et agroforesterie), l'Icipe (protection des plantes) et l'Ilri (élevage et marché). S'appuyant sur le bilan de la période précédente - plus de 80 programmes de recherche, 100 étudiants encadrés, près de 180 copublications, une quarantaine d'évènements scientifiques - le nouvel accord reconnaît l'importance de la contribution française à la production de connaissances et de cadres scientifiques. II vise à renforcer les collaborations institutionnelles et scientifiques et à faciliter l'ouverture à l'Union européenne.

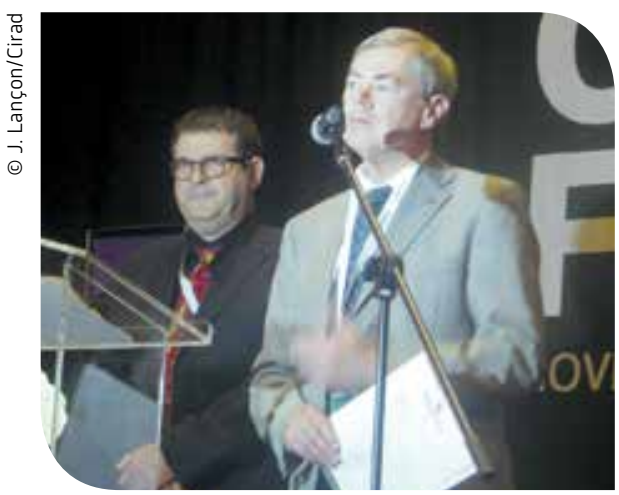

Patrick Caron, directeur de la recherche et de la stratégie, et David Radcliffe, Conseiller européen.

agricole d'Afrique, I'Union africaine, le Nepad et I'Union européenne. Patrick Caron a notamment pu s'entretenir avec ses partenaires du projet Prolntensafrica, qui vient de bénéficier d'un financement européen de deux ans pour élaborer un programme conjoint Europe-Afrique. Le Cirad a également participé à plusieurs sessions spéciales : Climate smart agriculture et Innovation platforms, amélioration de l'enseignement et des compétences en agriculture, et la plateforme Paepard (Platform for African - European Partnership in Agricultural Research for Development].

Dans le cadre du Centre for Study of Governance Innovation (Govlnn), le Cirad, partenaire de l'université de Pretoria, a contribué à l'organisation de la semaine sur l'innovation dans le domaine de la gouvernance du 2 au 7 juin. Vandana Shiva, activiste écologiste et féministe indienne, récipiendaire du prix Nobel alternatif, y a notamment donné une conférence intitulée Rethinking Development in the 21st Century.

jacques.lancon@cirad.fr, directeur régional Afrique australe et orientale

Un protocole d'accord entre le Cirad et le ministère malgache de l'Enseignement supérieur et de la Recherche scientifique (Mesupres) a été signé par Michel Eddi et la ministre Marie Monique Rasoazananera en juin. II précise les modalités de l'appui du Cirad à la politique de recherche malgache dans le cadre de programmes conjoints menés par des équipes mixtes de recherche.

pascal.danthu@cirad.fr, directeur régional Madagascar
Journées francomaghrébines de la recherche

\section{agronomique et vétérinaire}

La conférence a rassemblé le 31 octobre à Tunis les dirigeants des institutions de recherche agronomique et les responsables professionnels agricoles d'Algérie, de France, du Maroc et de Tunisie. Ce rendez-vous annuel a été initié en 2013 à Meknès. II a pour objectif de défınir des priorités de recherche et de partenariat entre pays méditerranéens sur « l'adaptation de l'agriculture méditerranéenne aux changements globaux ». Le Cirad tient notamment sa place en Afrique du Nord grâce au dispositif Sirma [Systèmes irrigués au Maghreb) et dans le cadre de projets financés par la l'Union européenne, I'Agence française de développement, l'Agence nationale de la recherche et la Fondation Agropolis.

\section{BILAN ET PERSPECTIVES}

DES ACTIONS MENÉES DEPUIS

\section{LE SÉMINAIRE DE MEKNĖs}

De nombreuses activités de recherche en partenariat sont en cours dans la région, notamment dans le cadre de projets menés sur financements européens (FP7, "Voisinage » ou Arimnet1], mais aussi de I'ANR ou l'AFD. Le dispositif en partenariat Sirma, animé par le Cirad, est au cœur de ces projets. Lancé en janvier, le programme Arimnet2 (coordination de la recherche agronomique en Méditerranée) implique 16 pays partenaires. Il est coordonné par I'Inra. L'IAV Hassan II, avec I'ANR-France, assure le secrétariat des appels à projets. Des collectifs franco-maghrébins sont déjà constitués pour la rédaction de propositions. D'autres actions France-Maghreb seront montées, notamment dans le cadre des appels à projets européens H2020 et EraNet-Med.

Enfin, dans le but de renforcer l'impact des recherches, les professionnels sont associés plus étroitement dans les projets. Des actions de formation seront menées à tous les niveaux. 


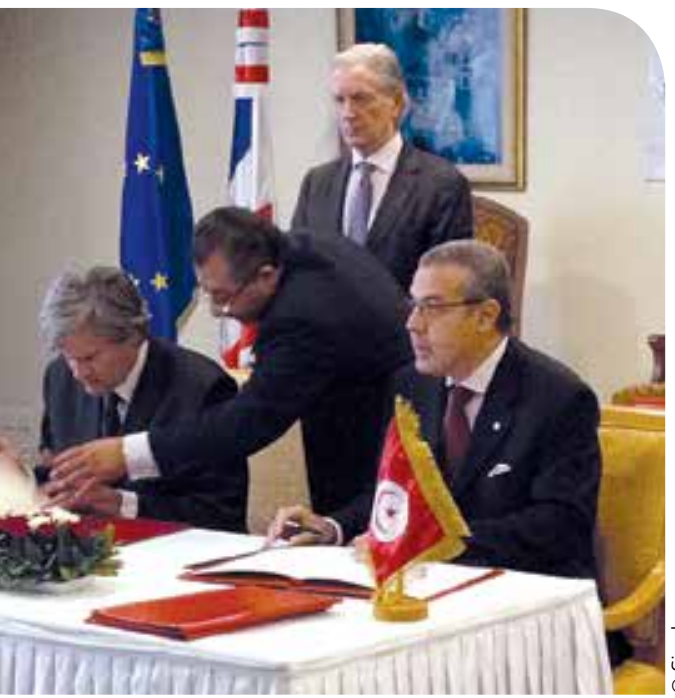

Ouverture de la conférence par MM. Stéphane Le Foll et Lassaad Lachaal, ministre tunisien de l'Agriculture.

\section{TROIS DOMAINES PRIORITAIRES DE RECHERCHE}

Les collaborations concernent prioritairement trois domaines.

> Santé animale-maladies vectorielles. Le réseau Remesa (Réseau méditerranéen de santé animale, FAO-OIE], qui rassemble 10 pays du pourtour méditerranéen, permettra d'animer le développement et la mise en œuvre de projets et programmes régionaux. L'UMR Cmaee (Cirad-Inra) et les instituts de l'Iresa et I'IAV-H2 montent des projets communs dans ce cadre.

$>$ Gestion de l'eau et des sols : les nombreuses actions menées par le dispositif en partenariat Sirma et le projet Sicmed (Inra, IRD) montrent l'existence d'une capacité collective à construire et mener à bien des projets de recherche.

$>$ Amélioration des plantes et des systèmes de cultures. De nombreux projets sont en cours (projet Arcade-Agropolis Fondation, thèses en cotutelle, mobilité Agreenskills]. Ils concernent notamment le blé dur, les fruitiers méditerranéens, le déficit hydrique, la tolérance au stress salin (agrumes, riz) et l'agriculture de conservation... Souvent bilatérales, ces collaborations pourraient être étendues dans un cadre multilatéral.

La prochaine conférence annuelle se tiendra en Algérie en 2015.

jean-luc.battini@cirad.fr Chargé de mission Méditerranée

\section{Des collaborations}

\section{franco-brésiliennes renforcées pour le développement durable de l'Amazonie}

Cinq ans après l'accord franco-brésilien sur le développement durable du Biome Amazonie et son premier séminaire, en 2009, le séminaire « Recherches et politiques publiques pour un développement durable en Amazonie » a été organisé les 11 et 12 décembre, à Brasilia. Près d'une centaine de participants, décideurs, bailleurs politiques et partenaires de recherche du Cirad (Dispositif en partenariat "Forêts, agriculture et développement territorial en Amazonie ") et de I'IRD [Programme pluridisciplinaire régional « Dynamiques environnementales, ressources et sociétés en Amazonie ») ont ainsi pu partager leurs visions, leurs modes d'intervention et réfléchir au futur.

Le séminaire a aussi permis de présenter le programme franco-brésilien Guyamazon, dont l'objectif est de soutenir des projets de recherche, de formation et d'innovation conjoints et de contribuer à la consolidation des collaborations entre la Guyane et les États amazoniens du Brésil. Le programme initié en 2012 regroupe 132 chercheurs de 32 institutions et envisage de s'élargir à d'autres partenaires. II réunit I'Ambassade de France, la Région Guyane, I'IRD, le Cirad et les fondations d'appui à la recherche des États d'Amazonas, d'Amapá, et du Maranhão.

bernard.mallet@cirad.fr

Directeur régional Brésil

En savoir plus sur le biome amazonien

http://www.accord-biome-amazonien.org

En savoir plus ur Guyamazon

http://www.ird.fr/les-partenariats/programmes-multilateraux/guyamazon

Frédéric Huynih (IRD), Doris Sayago (CDS/UNB) et Bernard Mallet, directeur régional du Cirad au Brésil ouvrent le séminaire à Brasilia. @ Cirad

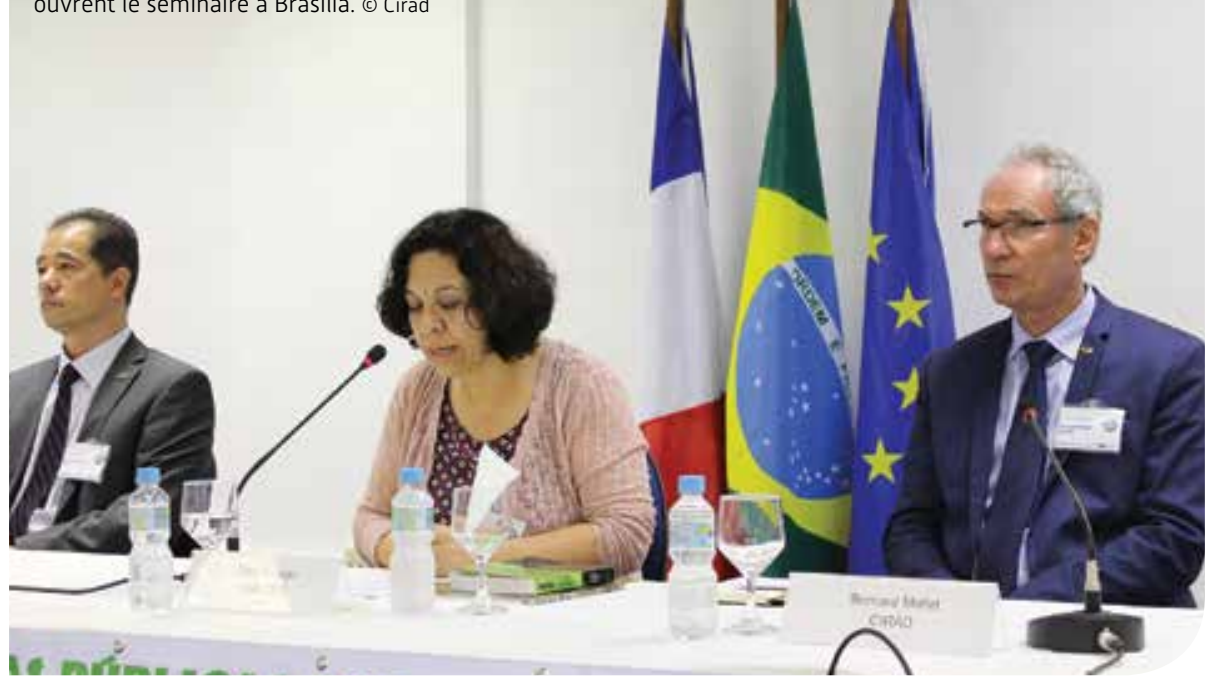




\section{La santé animale en Asie}

LA PLATEFORME RÉGIONALE DE RECHERCHE-ASIE (PRR-ASIE), bâtie sur le site de l'Institut Pasteur du Cambodge (IPC), a été inaugurée officiellement en mars par le ministre de la Santé du Royaume du Cambodge, SE le PrMAM Bun Heng, en présence des ambassadeurs de France et des pays asiatiques et de personnalités nationales et internationales de la santé. Cette plateforme a été réalisée par l'Alliance nationale française pour les sciences de la vie et de la santé (Aviesan Sud), dont le Cirad est membre associé. L'inauguration a été suivie d'un symposium international sur les maladies infectieuses émergentes en Asie du Sud-Est.

LE PROJET COMACROSS, Companion Approach for Cross-sectoral collaboration in health risks management in SEA, financé par I'Union européenne et coordonné par le Cirad, a été lancé. Il vise à renforcer les capacités, en particulier, des pays les plus vulnérables d'Asie du Sud-Est, à prévenir et gérer les risques liés aux maladies infectieuses émergentes. Le projet associe l'université Kasetsart (KU), I'Institut Pasteur au Cambodge (IPC), I'université d'Oxford, Lao-Oxford Mahosot Wellcome Trust Research Unit (LOMWRU) et la National University of Laos (NUoL).

philippe.girard@cirad.fr

Directeur régional Asie du Sud-Est continentale

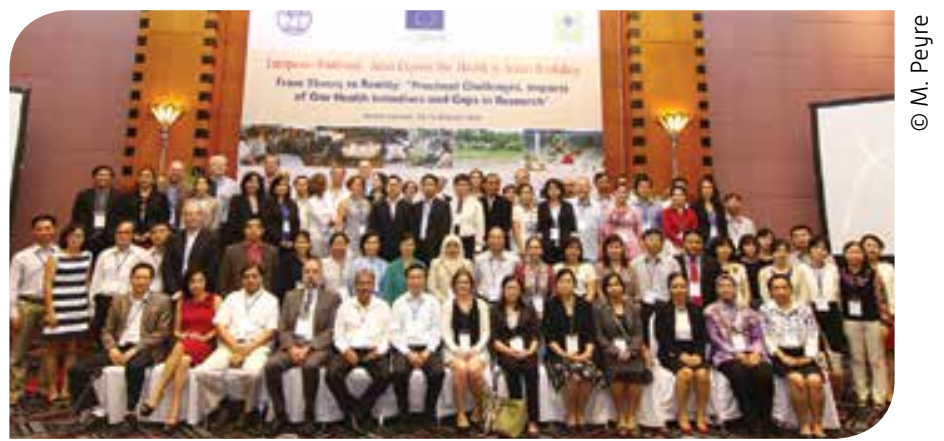

UN NOUVEAU MASTER INTERNATIONAL, INTERRISK, sur l'évaluation et la gestion des risques infectieux à l'interface de l'homme, de l'animal et des écosystèmes (InterRisk), est en création en Thaïlande. Il est le fruit de la collaboration entre la Faculté vétérinaire de Kasetsart, l'Ėcole vétérinaire de Toulouse [ENVT] et le Cirad. Les enseignements seront dispensés en Thaïlande à Bangkok, à l'université de Kasetsart, partenaire du Cirad. Ce master pourra déboucher sur un PhD programme en sciences vétérinaires à l'université de Kasetsart. II se fera en lien avec Agreenium dans le cadre du réseau Grease.

LE RÉSEAU SEA EU NET a pour objectif de renforcer la coopération scientifique entre l'Europe et l'Asie. L'atelier d'experts "One health in Action » qui s'est tenu à Hanoi avec de nombreux partenaires, en octobre, a ainsi permis d'identifier les besoins en recherche en Asie du Sud-Est dans le domaine de la surveillance sanitaire. Le réseau est aussi à l'origine d'une Master class «One health » : 10 jours en France au Cirad en octobre, qui seront suivis d'un séjour de 10 jours en 2015 en Thaïlande. Cette Master class permet à 12 jeunes scientifiques de compétences et d'horizon diversifiés de développer conjointement leur projets de recherche, avec l'ambition de favoriser les approches pluridisciplinaires.

flavie.goutard@cirad.fr Animal et gestion intégrée des risques [UR AGIRs]

http://sea-eu.net

\section{Palmier à huile et environnement. Le défı de la durabilité}

La conférence Icope, International Conference on Oil Palm and the Environment s'est tenue en février, à Bali, sous la bannière : « La culture du palmier à huile : devenir un modèle pour l'agriculture durable de demain ? ». Pour la $4^{\mathrm{e}}$ fois depuis 2007, le Cirad en est l'organisateur, avec la société indonésienne PT Smart et I'ONG WWF Indonésie.

Depuis plusieurs décennies, sous la pression de la société civile et des ONG, les acteurs de la filière transforment en profondeur les systèmes d'exploitation, génèrent des critères normatifs de durabilité et investissent dans de grands projets de recherche internationaux. Ces efforts ont pour objectif de mieux connaître les déterminants de la durabilité et les interactions entre espaces naturels et plantations. Grâce à son expertise et sa connaissance du terrain, le Cirad est fortement impliqué dans ces réflexions. Les projets en partenariat Spop (production durable d'huile de palme) et Prigoue (rôles des standards privés multi-acteurs dans la gouvernance globale de l'environnement), soutenus par l'Agence nationale de la recherche, y contribuent.

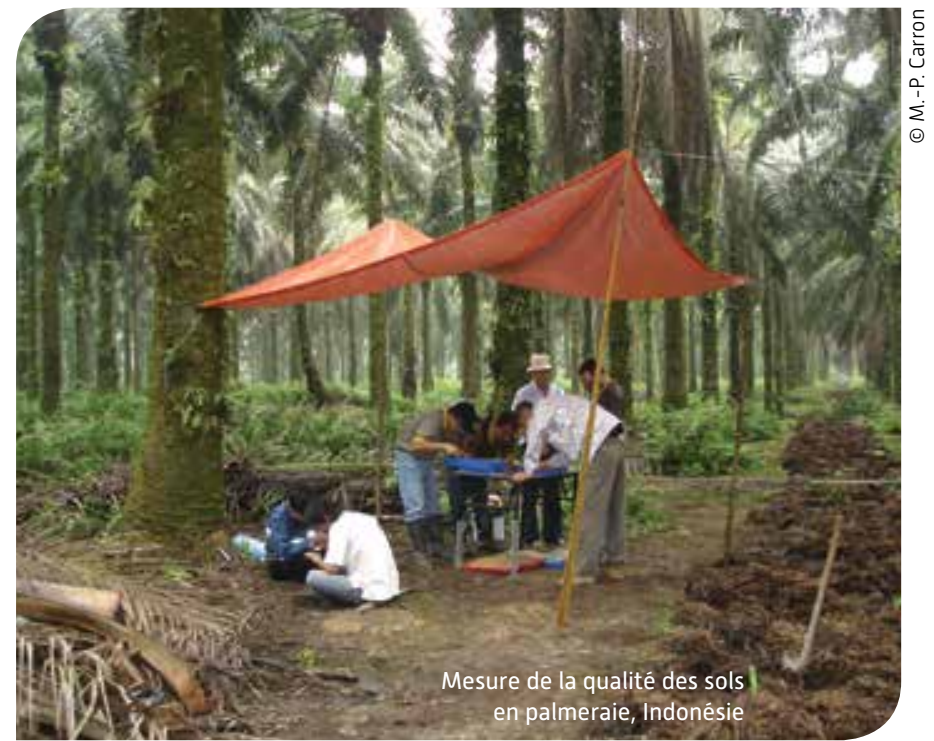




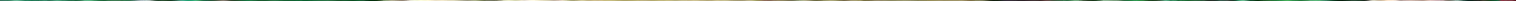

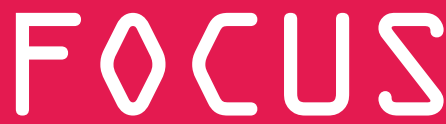

\section{sur l'année}

\section{AGRICULTURES}

FAMILIALES
A Madagascar, la récolte du clou de girofle est une activité familiale. La petite fille accompagne ses parents dans les vergers et assiste à la récolte. E. Malézieux Cirad

Consultez notre dossier complet sur les agricultures familiales :

www.cirad.fr

onglet $>$ Nos recherches rubrique $>$ Thèmes de recherche

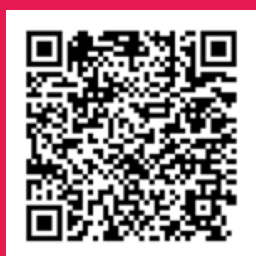


2014 a été déclarée

Année internationale

de l'agriculture

familiale (AIAF)

par les Nations unies.

Améliorer

les conditions de

production et de vie

des agricultures

familiales est une

mission prioritaire pour

le Cirad. Grâce

à ses nombreuses

actions de

communication,

en France et

dans le monde,

il a développé et fait

valoir son expertise

tout au long de l'année

2014 sur ce thème

qui lui est cher.

Depuis 2012, Jean-Michel Sourisseau, socio-économiste, coordonne les travaux du Cirad sur l'agriculture familiale pour la préparation de l'année internationale. Il fait ici un bilan de l'année écoulée.

L'année 2014 a été déclarée ANNÉE INTERNATIONALE DE L'AGRICULTURE FAMILIALE par I'organisation des Nations unies. De par son mandat, le Cirad a largement contribué à afficher la présence française sur cette thématique. Quel bilan l'établissement tire-t-il de cette année ?

II s'agissait d'une opportunité extraordinaire de valoriser notre savoir-faire, tant en matière de production scientifique qu'en termes de communication internationale. En ce qui concerne les publications, le bilan 2014 était de 26 articles, 6 ouvrages, 17 chapitres d'ouvrages et pas moins de 18 communications référencés. Un des moments forts a été l'organisation des Rencontres internationales "Agricultures familiales et recherche » avec Agropolis, le $G_{F A R}{ }^{1}$, le CGIAR $^{2}$ et le $F R M^{3}$, seul événement de recherche dans l'agenda officiel de l'Année Internationale. Nous avons réussi à être également très présents auprès du grand public, qui a particulièrement été interpellé par ce thème. Au Salon international de l'agriculture, nous avons organisé débats et conférences sur un stand de $200 \mathrm{~m}^{2}$ réalisé en partenariat avec I'AFD et France Médias Monde. L'exposition itinérante que nous avons conçue avec I'AFD $^{4}$, I'Institut français et l'IRD $^{5}$ a fait le tour du monde dans ses différentes versions linguistiques et a très largement circulé en France via les conseils généraux, municipalités et lycées agricoles.
Comment expliquez-vous

ce large intérêt pour

une thématique qui semble,

a priori, tournée vers les pays

du Sud?

C'est loin d'être une thématique " des Suds » comme on le croit à tort. En France, 80 \% des exploitations agricoles relèvent de l'agriculture familiale et les jeunes agriculteurs français interrogent les limites du modèle actuel en lien avec la notion d'agriculture familiale. A l'échelle de la planète, la société civile se soucie davantage de l'origine des produits qu'elle consomme et s'inquiète des risques d'une industrialisation agricole et de ses conséquences économiques, sociales et environnementales. La médiatisation de ces préoccupations remonte de toute évidence aux émeutes de la faim de 2008. Elles ont révélé l'importance de la financiarisation de l'agriculture. Cela a largement contribué à l'ouverture de discussions sur les modèles de production et à l'intérêt des opinions publiques de nombreux pays.

\section{Comment appréciez-vous}

le rapport sur I'AIAF 2014 remis par Henri Rouillé d'Orfeuil ${ }^{6}$

à Stéphane Le Foll

et Annick Girardin ?

Etant donné la place faite au Cirad dans ce rapport, nous ne pouvons qu'en être enchantés. La qualité de nos publications et de nos actions y sont mentionnées. Notre rôle de relais vers les organisations internationales de recherches agricoles telles que le CGIAR

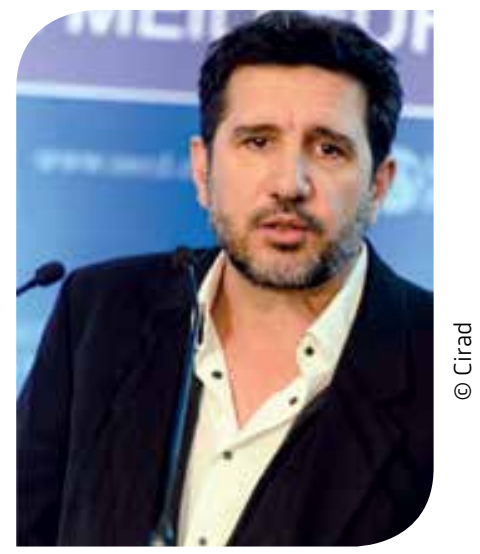

ou le GFAR y est souligné... C'est une reconnaissance de la qualité de notre positionnement sur cette thématique.

\section{Et... après 2014 ?}

Les enjeux sont nombreux : avec nos partenaires français (IRD, Inra, Agreenium...., au-delà des préconisations nationales et internationales auxquelles nous allons répondre. En effet, la question de la constitution et de I'animation d'un réseau scientifique et universitaire "agriculture familiale » nous a été soumise. Nous pensons par ailleurs que l'évolution des politiques agricoles et la prise en compte de la spécificité de cette forme de production sont des enjeux importants tant en Europe que dans les pays qui accueillent nos recherches. Nous sommes donc toujours mobilisés sur les agricultures familiales et adhérons à l'idée du Forum Rural Mondial de prolonger les efforts fournis sous la forme d'une décennie de l'agriculture familliale.

1. GFAR : Global Forum on Agricultural Research.

2. CGIAR : Consultative Group for International Agricultural Research.

3. FRM : Forum rural mondial.

4. AFD : Agence française de développement.

5. IRD : Institut de recherche pour le développement.

6. Coordinateur de I'AIAF en France. 


\section{Des avancées maje
pour la recherche}

Pour la recherche, quatre résultats clés sont à retenir.

$>$ L'agriculture familiale prend sa place comme catégorie sociopolitique

Une défınition se dégage : est familiale une agriculture mise en œuvre par des exploitations agricoles mobilisant le travail de membres de la famille, mais pas de salariés permanents. Les nombreuses discussions autour du critère choisi (pas de salarié permanent), qui décrit l'imbrication entre famille et exploitation, ont mis en évidence l'existence d'un continuum de situations, depuis la petite exploitation familiale jusqu'à l'entreprise agricole capitaliste.

$>$ C'est une porte d'entrée dans la contestation d'un modèle agricole 《conventionnel》

Introduire la famille dans la réflexion sur les modèles agricoles invite à discuter autrement les nombreux défis auxquels l'agriculture est confrontée :

- Développer l'emploi et lutter contre la pauvreté.

- Assurer la sécurité alimentaire.

- Concilier production et environnement.

- Valoriser les territoires ruraux.

- Faire face aux risques sanitaires.

- Lutter contre le changement climatique.

- Favoriser la transition énergétique.

- Renforcer les solidarités.

$>$ L'agriculture familiale un objet de recherche

Des résultats majeurs ont été démontrés:

- Les agricultures familiales peuvent nourrir le monde et approvisionner les marchés; elles produisent aujourd'hui bien plus, en volume et valeur, que les fermes industrielles.

- Les pratiques et les performances de la plupart des fermes du monde dépendent des liens entre sphères domestiques et productives.

- La production agricole peut être abordée dans une perspective plus globale de développement.

\section{$>$ L'agriculture familiale soulève aussi des questions}

- Comment mesurer les performances en agriculture ? Quelles nouvelles pratiques, quels modèles alternatifs, quel accompagnement adapter aux logiques familiales?

- Quelles données collecter et analyser pour identifier vraiment le poids et l'importance des différentes formes d'agriculture ?

- Quelles politiques publiques déployer : moins sectorielles, plus diverses et plus articulées?
En 2050, l'agriculture devra nourrir 9 milliards de personnes. Quelle place occupera alors l'agriculture familiale?

jean-michel.sourisseau@cirad.fr

Acteurs, ressources et territoires dans le développement. (UMR Art-Dev)

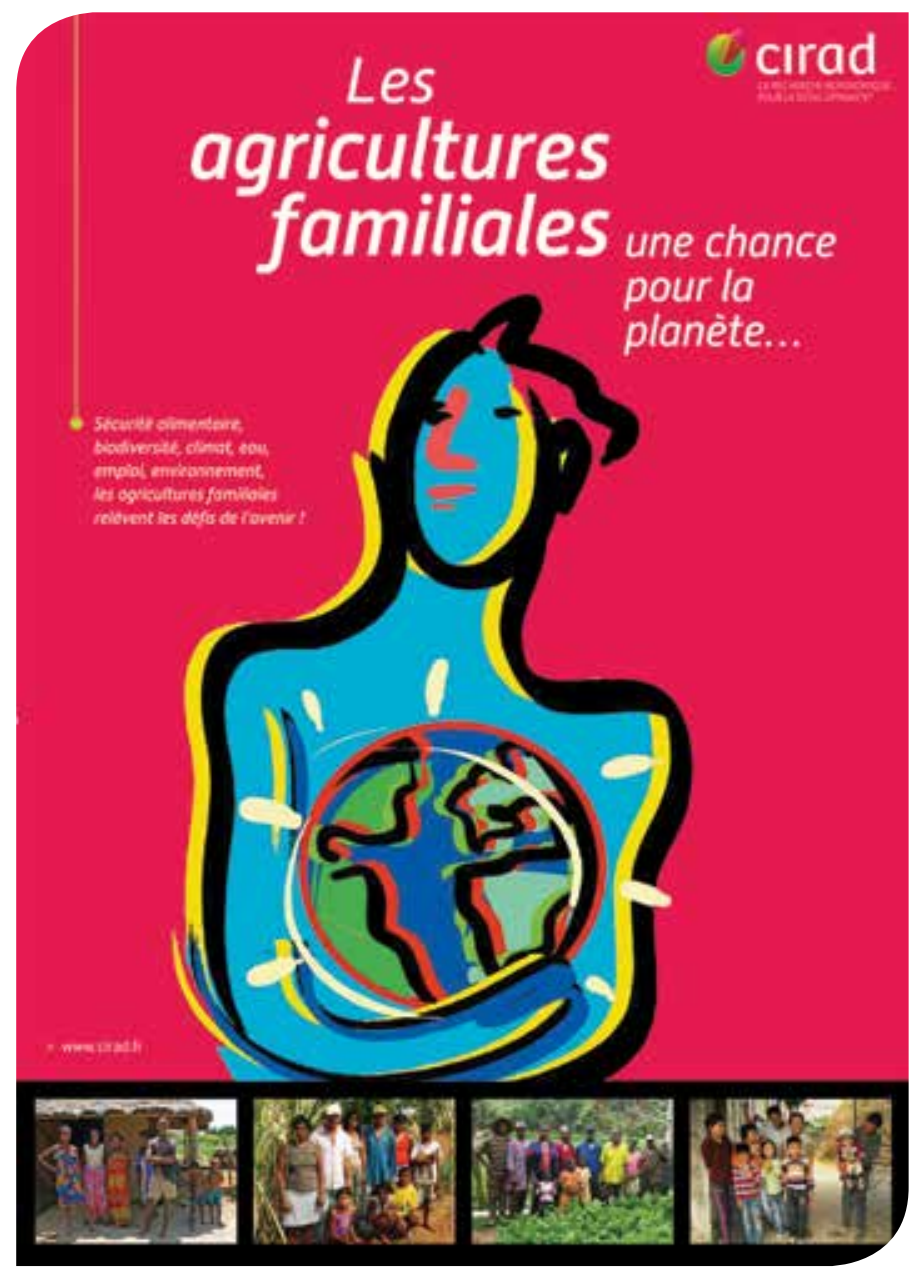

己っb milliards de personnes produisent près de $80 \%$ de la production alimentaire mondiale sur plus de 500 millions d'exploitation. 


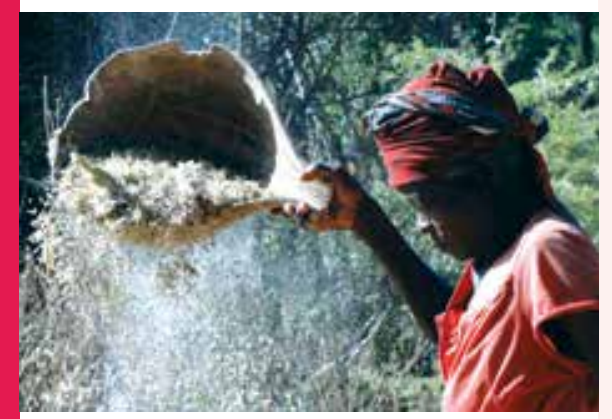

() Labeyrie V. / Cirad

PARTENAIRES

France $>$ Institut de recherche pour le développement (IRD) ; Université d'Aix-Marseille. Kenya > National Genebank of Kenya (Kari]

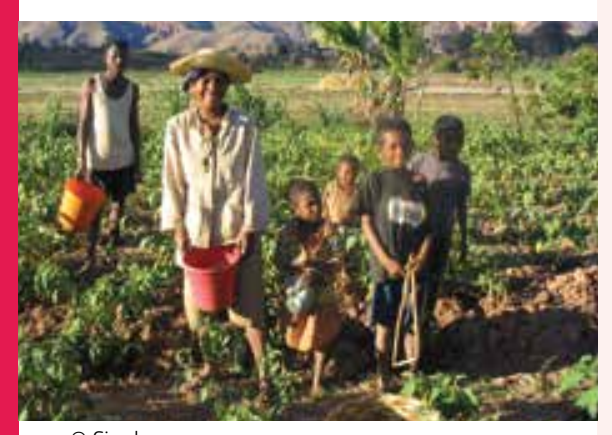

(ㄷ) Cirad

PARTENAIRE

France $>$ Centre d'études et de recherches sur le développement international (Cerdi)
DIVERSITÉ DES PLANTES CULTIVÉES L'organisation sociale comme facteur déterminant

Comment les sociétés humaines influent-elles sur les processus d'évolution des plantes cultivées ? Les chercheurs ont démontré que la distribution de la diversité génétique du sorgho dans l'est du Kenya était liée à l'appartenance ethnique des agriculteurs. Leurs résultats montrent, en effet, que la distribution spatiale de la diversité du sorgho, tant du point de vue des variétés identifiées par les agriculteurs que du point de vue génétique, est associée à l'organisation ethnique. Si les variétés introduites, issues du système formel d'amélioration variétale, sont distribuées de façon uniforme dans les trois groupes ethniques, plusieurs variétés locales identifiées par les agriculteurs sont en revanche réparties de façon inégale entre les groupes. En intégrant cette dimension sociale, les programmes de collecte et de caractérisation des ressources génétiques gagneraient en efficacité. C'est là l'enjeu majeur pour la conservation de ces ressources.

vanesse.labeyrie@cirad.fr, Amélioration génétique et adaptation des plantes méditerranéennes et tropicales (UMR Agap)

PLoS One, 9 : e92178. Doi : 10.1371/journal.pone.0092178

\section{RÉSILIENCE, VULNÉRABILITÉ ET DURABILITÉ Comment évaluer les systèmes agricoles}

A Madagascar, dans la région du lac Alaotra, les exploitations agricoles sont confrontées à une multitude de risques. Quelles sont alors leurs capacités d'innovation et d'adaptation? Sur quels critères analyser leur vulnérabilité, leur résilience, leur durabilité ? Les chercheurs ont sélectionné un certain nombre d'indicateurs socio-économiques pertinents à l'échelle du système d'activité que constituent le ménage et son exploitation agricole. Grâce à ces indicateurs, ils ont mis en évidence une forte résilience globale des exploitations agricoles face aux aléas climatiques et socio-économiques. Cette résilience tient à une large diversification des activités, y compris des activités non agricoles. Elle est aussi due à une capacité d'innovation technique relativement forte des systèmes de culture et d'élevage, qui résulte de la présence historique de nombreux projets de développement dans la région. Cette étude constitue une étape dans la construction d'un observatoire des agricultures du monde.

eric.penot@cirad.fr, Innovation et développement dans l'agriculture et l'agroalimentaire [UMR Innovation]

helene.david-benz@cirad.fr, Marchés, organisations, institutions et stratégies d'acteurs (UMR Moisa)

Ethique et économique, $11: 44-61$.

papyrus.bib.umontreal.ca/xmlui/bitstream/handle/1866/10261/Penot\%20et\%20al.-11\%281\%29.pdf

\section{ELEVAGE EN EGYPTE Un facteur d'adaptation au changement global}

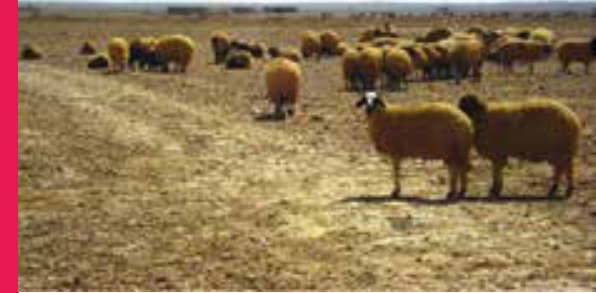

(c) Elvulmed / Cirad

PARTENAIRES

Egypte > Animal Production Research Institute (Apri) ; Desert Research Center [DRC] ; Gouvernorat de

Matrouh ; International Center for Agricultural Research in the Dry Areas (Icarda). France > Institut national de la recherche agronomique (Inra]
Le bassin méditerranéen est confronté à des changements sociaux et environnementaux sans précédent qui fragilisent ses territoires. L'élevage, qui y a toujours été un élément structurant de l'espace et de l'économie des ménages, peut-il jouer un rôle dans la résilience de ces régions ? Quelles sont ses capacités d'adaptation au changement global ? Un projet de recherche, coordonné par le Cirad, a apporté des réponses à ces questions pour les régions côtières de l'Egypte soumises pendant une quinzaine d'années à une sécheresse exceptionnelle. Ses résultats mettent en évidence le rôle central de l'organisation sociale tribale dans l'accès et l'usage des ressources, notamment foncières, mais aussi dans l'adaptation des familles. Ils pointent aussi la capacité des Bédouins à adopter de nouvelles activités, entre autres grâce à la migration, et le rôle de cette diversification dans l'incidence de la sécheresse sur leurs conditions de vie. Cependant, malgré la multitude des formes de diversification, l'élevage reste une composante essentielle de la société : le bétail a toujours un rôle d'assurance sociale, pour les Bédouins qui ont migré en ville ou à l'étranger, et économique, pour ceux qui sont restés en milieu rural.

veronique.alary@cirad.fr, Systèmes d'élevage méditerranéens et tropicaux (UMR Selmet]

jean-francois.tourrand@cirad.fr, Gestion des ressources renouvelables et de l'environnement (UPR Green) Agricultural Systems, 128 : 44-54. Doi : 10.1016/j.agsy.2014.03.008 


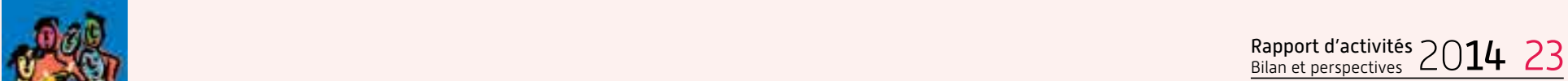

\section{PUBLICATIONS}

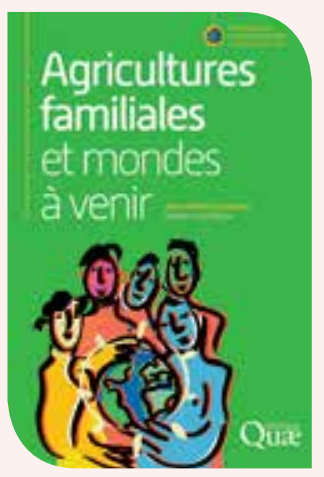

\section{Agricultures familiales et mondes à venir}

Cet ouvrage, issu d'un travail collaboratif conduit par le Cirad, consacre trois décennies de recherches sur ce sujet. II revisite les approches les plus utilisées pour analyser et comprendre l'agriculture familiale qui est au cœur des agricultures du monde. II est primordial de bien définir l'agriculture familiale pour mieux en cerner les enjeux, la replacer dans un contexte plus global et ainsi juger de sa contribution à un développement qui soit durable et plus équitable. Cette forme de production est ainsi au centre des débats sur le développement agricole.

J-M. Sourisseau [coord.), 2014. Agricultures familiales et mondes à venir. Collection Agricultures et défis du monde, Cirad-AFD. Editions Quae.
Et pour aller plus loin...

J.-F. Bélières, Ph. Bonnal, P.-M. Bosc et al. [coord.), 2014. Les agricultures familiales du monde: définitions, contributions et politiques publiques. Collection A savoir, Cirad-AFD.

http://www.afd.fr/home/publications/travaux-de-recherche/publications-scientifiques/a-savoir

Bosc P.M. (ed.), Sourisseau J.M. (ed.), Bonnal P. (ed.) et al. 2015. Diversité des agricultures familiales: Exister se transformer, devenir. Versailles: Ed. Quae. [20150212]. http://publications.cta.int/media/publications/downloads/1839_PDF_PYW9OuW.pdf

Bois et forêts des tropiques $\mathrm{N}^{\circ} 319$. Spécial Agriculture familiale. Tous les articles sont gratuitement accessibles sur le site web de la revue http://bft.cirad.fr/

Perspective $n^{\circ} 27$. Production durable de cacao : s'inspirer de l'agroforesterie.

Perspective $n^{\circ} 29$. Politiques publiques pour l'agriculture familiale : caractériser pour mieux soutenir.

Pour télécharger les numéros de Perspective

http://www.cirad.fr/publications-ressources/edition/perspective-policy-brief

\section{Mieux valoriser les agricultures familiales en Amérique latine}

Du 2 au 5 novembre 2014 à Santiago du Chili, l'agriculture familiale en Amérique latine était au centre de l'attention des décideurs et spécialistes des politiques publiques. Le Cirad, la Cepal, la FAO, le FIDA, le ministère de I'Agriculture du Chili et I'IICA y ont organisé le séminaire international "Políticas públicas y agricultura familiar en América Latina y el Caribe ». Son objectif était d'établir un état des lieux des politiques publiques touchant les agricultures familiales dans cette région du monde et d'en définir les défis et les perspectives pour les quinze prochaines années.

\section{eric.sabourin@cirad.fr}

Acteurs, ressources et territoires dans le développement [UMR Art-Dev], animateur du dispositif de recherche et d'enseignement en partenariat PP-AL

www.pp-al.org

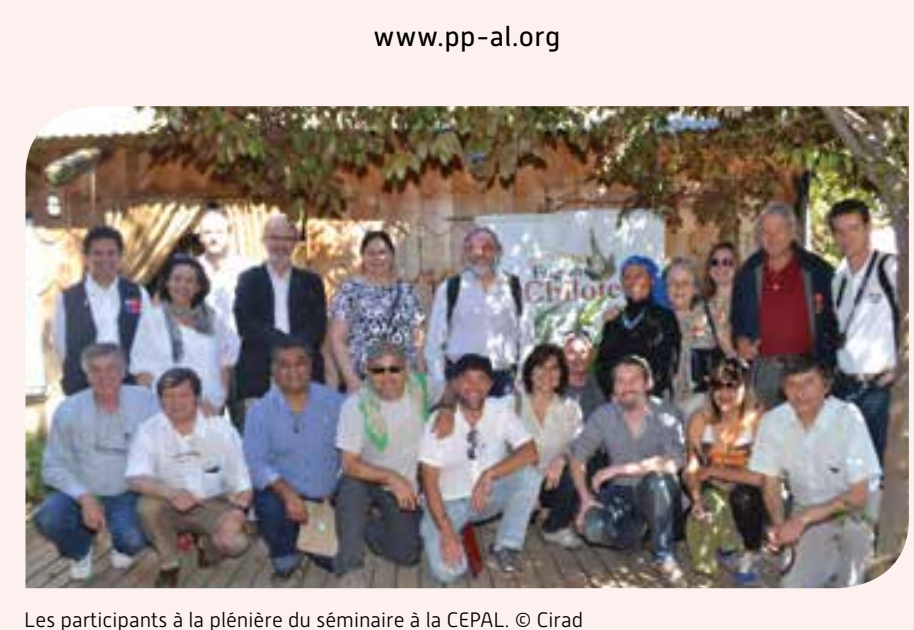

Les participants à la plénière du séminaire à la CEPAL. @ Cirad

* Dans le cadre du dispositif de recherche et d'enseignement en partenariat « Politiques publiques et développement rural en Amérique latine (DP PP-AL).

Politicas públicas y agriculturas familiares en América Latina y el Caribe: balance, desafios y perspectivas. Coord. E. Sabourin, M. Samper, O. Sotomayor. Coed. Cepal, Cirad, IICA, Red PP-AL. 2014

\section{En Amérique latine,}

\section{4 millions d'exploitations agricoles sont de type familial}




\section{RENCONTRES / ÉVÉNEMENTS}

\section{$1^{\text {res }}$ Rencontres internationales \\ LE LAIT, \\ VECTEUR DE DÉVELOPPEMENT}

Outre leur rôle nutritif majeur, le lait et les produits laitiers ont une forte dimension patrimoniale et culturelle. Dans les pays du Sud, ils jouent un rôle clé dans l'économie locale et impliquent souvent les femmes. Agreenium, dont le Cirad est membre fondateur, et le Consorzio Ricerca Filiera Lattiero Cascaria [CoRFiLaC) ont organisé les $1^{\text {res }}$ Rencontres internationales sur "Le lait, vecteur de développement » à Rennes, du 21 au 23 mai. Le partage d'expériences autour des défis techniques et sanitaires concernant le lait et sa transformation et la diversité des modèles laitiers observés furent au centre des échanges.

\section{bernard.faye@cirad.fr,}

Systèmes d'élevage en milieux méditerranéens et tropicaux [UMR Selmet]

\section{Rencontres internationales}

à Montpellier

\section{"Agricultures familiales} et recherche "

Du $1^{\text {er }}$ au 3 juin, Montpellier a accueilli plus de 250 agriculteurs, responsables d'organisations paysannes, responsables d'ONGs, décideurs politiques, agents de développement, chercheurs, citoyens engagés, venus de tous les continents pour réfléchir aux enjeux de recherche, sur, pour et avec les agricultures familiales. De nombreuses personnalités scientifiques ont contribué aux réflexions. Parmi elles, Hans Herren, président du Millennium Institute et lauréat du prix Nobel alternatif 2013 (" Right Livelihood Award ") a tenu une conférence auprès d'un large public sur le thème " L'agriculture familiale, une option crédible face aux défis de la planète ? ». Le Cirad a activement contribué à la préparation et au déroulement de cette conférence, organisée par Agropolis international en partenariat avec plusieurs organisations nationales et internationales.

http://www.agropolis.fr/actualites/2014retour-rencontres-internationales-agricultures-familiales-recherche.php
Agricultures familiales à Madagascar

UN ATOUT POUR LE DÉVELOPPEMENT DURABLE

C'était le thème de la semaine organisée par le Cirad et le Fofifa, avec le soutien de l'Union européenne, à l'université d'Antananarivo du 13 au 17 octobre. Des séminaires spécifiques ont été organisés pour les scientifiques, les organisations de producteurs et les politiques. L'exposition du Cirad sur les agricultures familiales a été ouverte pour 10 jours au public, et la journée mondiale de l'alimentation été fêtée dans ce cadre. Un document de synthèse et de valorisation des interventions et discussions a été produit.

pascal.danthu@cirad.fr, directeur régional Madagascar www.cirad.mg/aiafmada

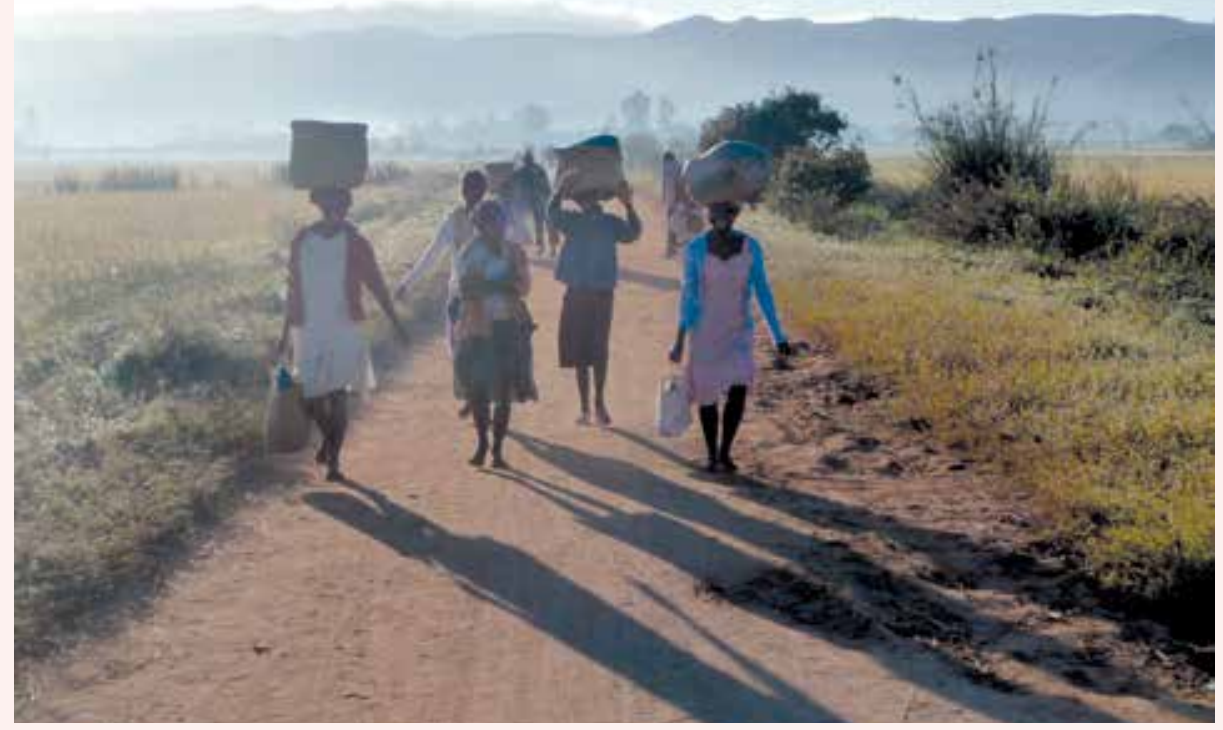

Paysannes partant au marché avec leur production dans la région du lac Alaotra, Madagascar. P.-Y. Le Gal @ Cirad

Congrès international AFTER 2014

\section{LES ALIMENTS TRADITIONNELS AFRICAINS VALORISÉS}

Le projet européen AFTER [African Food Tradition rEvisited by Research) vise à réunir l'Afrique et l'Europe autour d'un même objectif : améliorer les produits traditionnels africains à travers le partage et l'enrichissement des connaissances sur les technologies agroalimentaires. II œuvre à développer ou à créer un produit ou une technologie d'intérêt pour des entreprises agroalimentaires européennes ou africaines. Co-organisé par le Cirad, l'Ecole supérieure polytechnique (ESP-UCAD, Sénégal) et l'Association Afrique Agro Export [AAFEX], le premier congrès international du projet AFTER s'est tenu les 11 et 12 novembre à Dakar sur le thème "Valorisation des aliments traditionnels africains : innovations, qualité et accès au marché ". Il a rassemblé tous les acteurs de la filière agroalimentaire. Les résultats des recherches sur une vingtaine de produits traditionnels ont été présentés.

dominique.pallet@cirad.fr, Démarche intégrée pour l'obtention d'aliments de qualité (UMR Qualisud) www.after-fp7.eu/ 


\section{RÉSULTATS DE RECHERCHE}

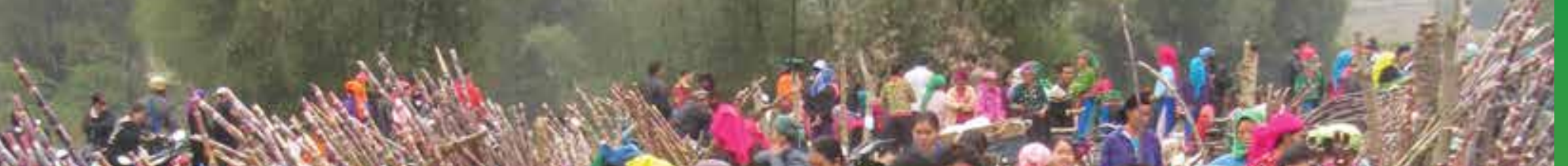

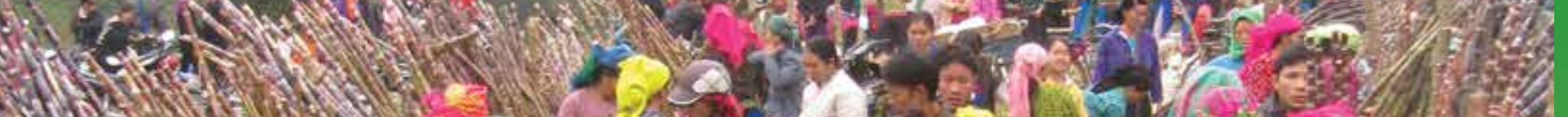

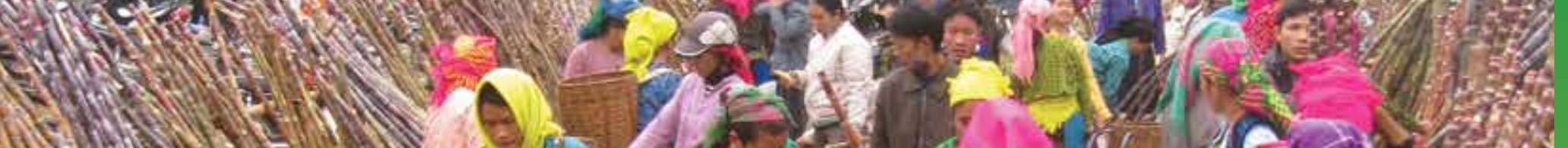

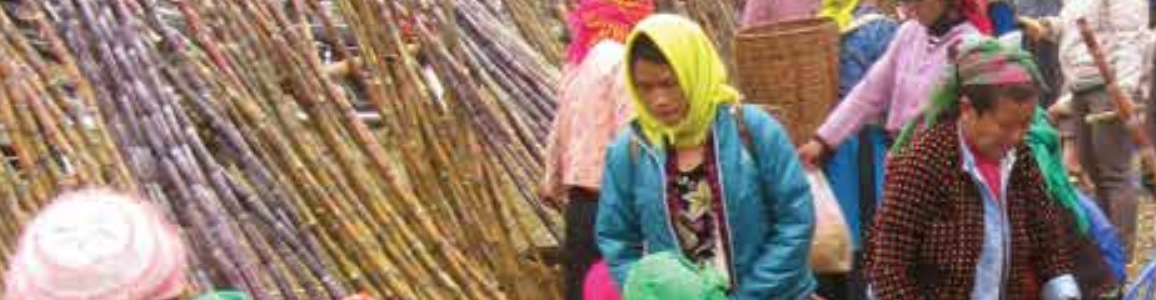

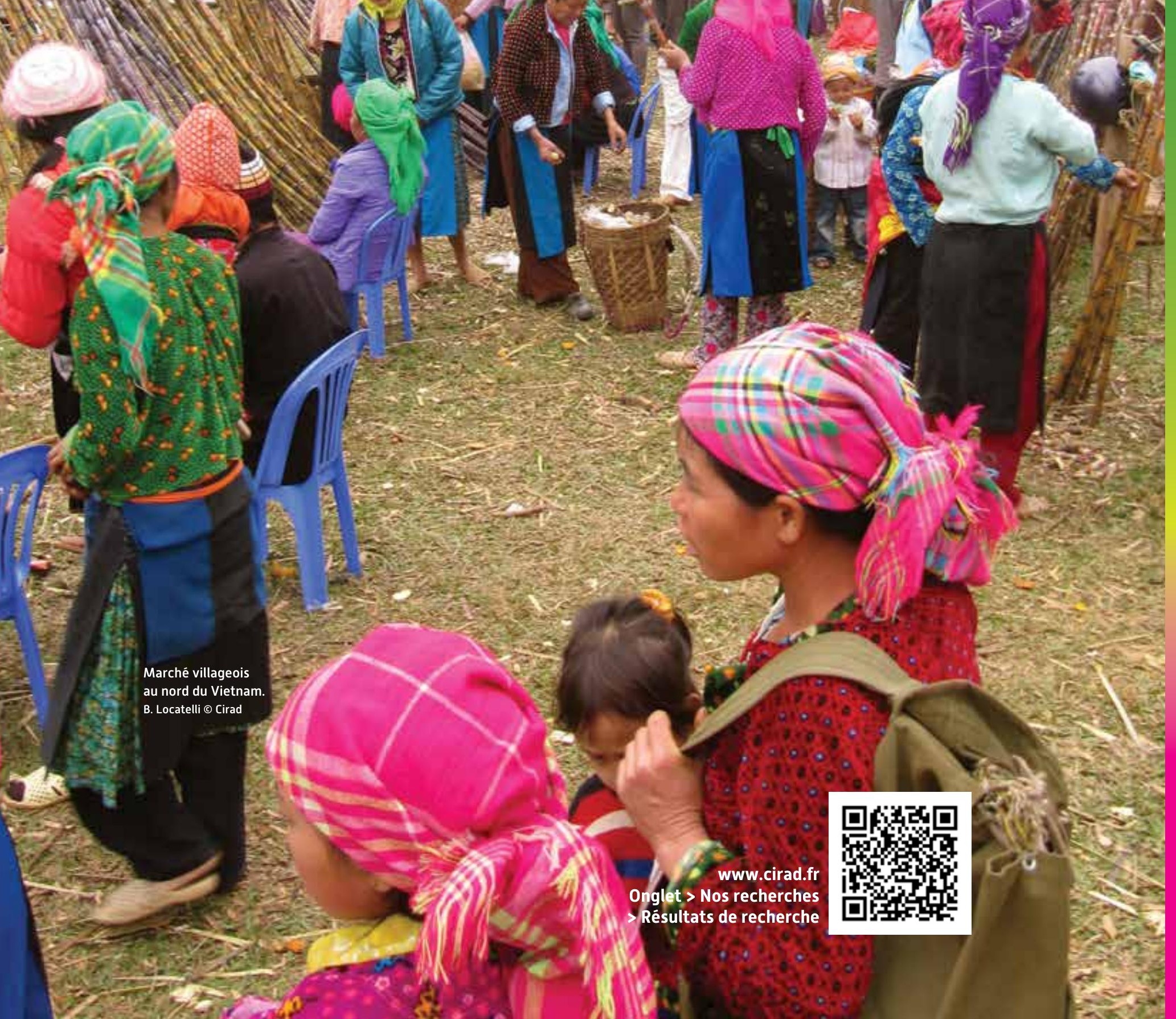




\section{AGRICULTURE ÉCOLOGIQUEMENT INTENSIVE}

\section{Transition vers l'intensification écologique LE RÔLE DE LA RECHERCHE SCIENTIFIQUE}

Le point de vue de François-Xavier Côte, directeur du département Performance des systèmes de production et de transformation tropicaux (Persyst]

QU'EST-CE QUE L'AGROÉCOLOGIE ET L'INTENSIFICATION ÉCOLOGIQUE POUR LE CIRAD ?

COMMENT CELA SE TRADUIT-IL EN TERMES DE THÉMATIQUES DE RECHERCHE?

LES RÉSULTATS SONT-ILS VISIBLES SUR LE TERRAIN?
Une famille très diversifiée de modèles de production agricole se réclame de l'agroécologie (agriculture de conservation, agriculture biologique, agroforesterie, etc.). Pour mieux concevoir et accompagner la transition agroécologique dans différents contextes au Sud, le Cirad a choisi de conduire prioritairement des recherches dans le champ de I'intensification écologique. Celle-ci vise à satisfaire la production tout en assurant une diversité de services écosystémiques en réduisant l'usage d'intrants de synthèse. L'intensification écologique est rendue possible en accroissant la biodiversité au sein des agrosystèmes et en favorisant les régulations naturelles des ravageurs, des maladies et l'efficience des cycles biogéochimiques.

Les recherches menées par le Cirad visent à mieux comprendre les mécanismes biophysiques, écologiques, économiques, sociaux et institutionnels qui régissent les agrosystèmes basés sur les principes de l'agroécologie. Le Cirad conduit non seulement des recherches sur l'étude des processus écologiques, mais également dans les domaines de la conception et de l'évaluation participative des systèmes de culture avec les agriculteurs, dans ceux de l'analyse et de l'accompagnement des systèmes d'innovation en appui à la transition écologique à différentes échelles, en particulier territoriales. C'est un positionnement scientifique original, il nous permet d'être plus pertinents dans nos recherches en réunissant les connaissances produites des 3 départements et en étant directement engagé sur le terrain dans les processus d'innovation.

Oui, et j'espère qu'ils le seront de plus en plus compte tenu de l'investissement du Cirad dans les recherches en agroécologie et intensification écologique. Il y a déjà de très belles réalisations concrètes dans les Dispositifs de recherche en partenariat (DP). On peut par exemple citer ce qui est fait à Madagascar sur les cultures de riz d'altitude, en Asie du Sud-Est et en Afrique de l'Ouest et du centre sur les cultures vivrières, en Afrique et en Amérique centrale sur l'agroforesterie, dans les DOM sur les cultures fruitières et maraîchères,...

Le rôle de la recherche est essentiel, il faut poursuivre dans une action qui combine production de connaissances et réalisations pratiques sur le terrain avec les producteurs et les acteurs du développement. Il faut résolument continuer à développer les connaissances sur les processus écologiques et biophysiques en jeu dans les différents contextes de production du Sud, poursuivre les études sur la gamme d'options techniques possibles et les tester et les évaluer. La recherche doit continuer à accompagner les acteurs dans la transition écologique à l'échelle des exploitations mais également et de façon essentielle à l'échelle des territoires. Cela doit permettre de développer l'action collective, les dispositifs d'accompagnement, l'information et l'implication des politiques publiques, bref de se donner toutes les chances de réussir et d'avoir un impact sur le terrain.

\section{Déchets organiques \\ Une mine pour \\ l'agriculture?}

Sur l'île de la Réunion, la fertilisation actuelle de la canne à sucre repose largement sur l'importation d'engrais minéraux et de synthèse. Les agriculteurs sont ainsi soumis à la disponibilité et à la volatilité des prix de ces engrais. Face à l'épuisement global des ressources en phosphore, les déchets organiques d'origine animale, urbaine ou agro-industrielle constituent de véritables mines du futur pour la fertilisation des cultures. Le Cirad vient de lancer une étude de fond sur la fertilité en phosphore des sols de la Réunion et I'entretien de cette fertilité via l'épandage de différents types de déchets organiques. Des premiers résultats sont attendus dès 2015.

cecile.nobile@cirad.fr matthieu.bravin@cirad.fr

(Recyclage et risque)

http://sps2014.cirad.fr/

La canne à sucre occupe près de $60 \%$ des surfaces cultivées de la Réunion. 


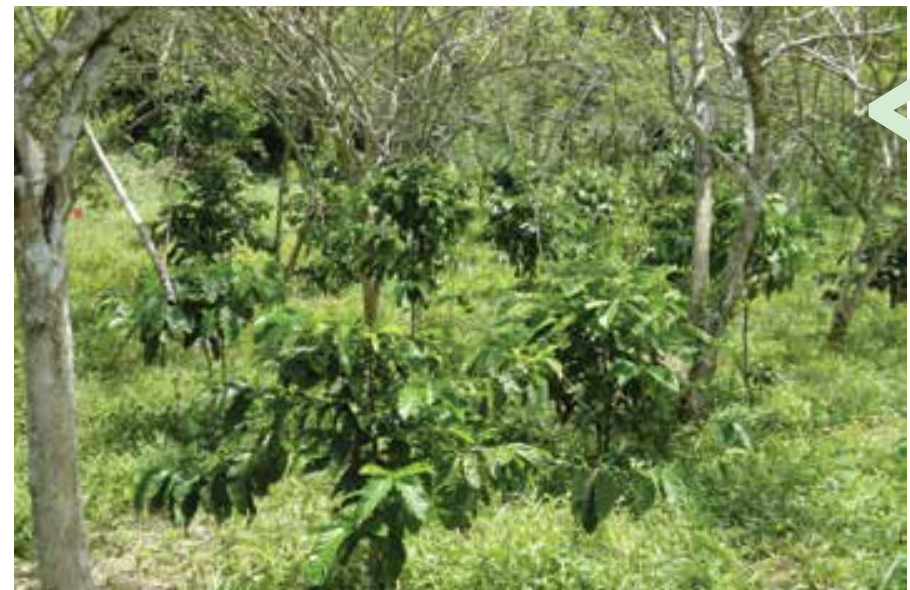

La collection de caféiers Coffea canephora conservée en Guayne. ๑ T. Leroy/Cirad

\section{PARTENAIRES}

Côte d'Ivoire $>$ Centre national de recherche agronomique (CNRA).

Equateur > Instituto Nacional de Investigaciones Agropecuarias (Iniap).

France $>$ Institut national de la recherche agronomique (Inra].

Ouganda > National Agricultural Research Organisation - Coffee Research Centre (Naro-Corec).

République démocratique du Congo > Université de Kinshasa.
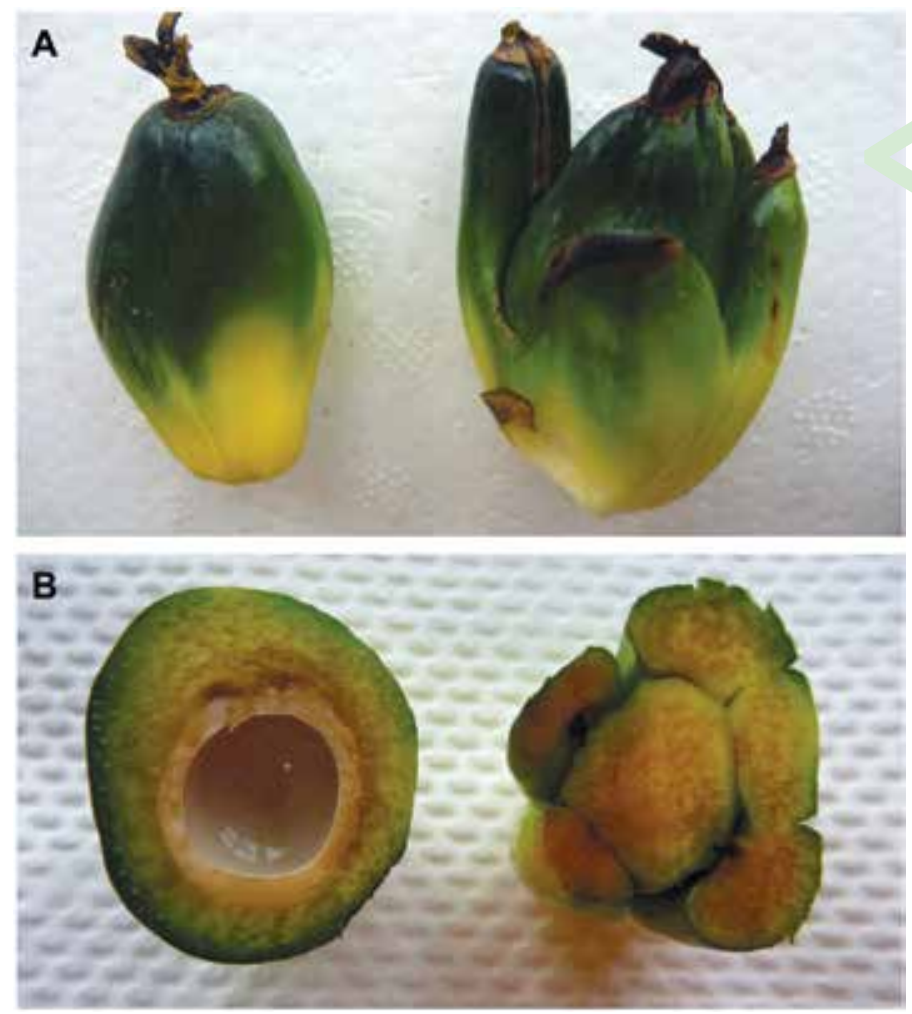

A. Fruits entiers de palmiers à huile : normal, à gauche, mantled, à droite. II s'agit ici de la variété virescens dont les fruits sont vert vif jusqu'à maturité B. Fruits en coupe transversale : normal, à gauche, mantled, à droite. II s'agit ici de la variété virescens dont les fruits sont vert vif jusqu'à maturité. @ A. Rival/Cirad

\section{CAFÉIER}

\section{DES COLLECTIONS DE RÉFÉRENCE POUR MIEUX EXPLOITER LA DIVERSITÉ}

thierry.leroy@cirad.fr (Amélioration génétique et adaptation des plantes méditerranéennes et tropicales - UMR Agap]

Les chercheurs du Cirad et leurs partenaires ont constitué plusieurs collections de référence de caféiers Coffea canephora, à partir d'une analyse de la diversité génétique de l'espèce. Ces petites collections, plus faciles à gérer et à maintenir que les vastes collections actuellement conservées par les centres de recherche, sont des outils précieux pour les sélectionneurs. Elles devraient leur permettre de faire face aux nouveaux défis auxquels sont confrontés les producteurs de café, qu'ils soient parasitaires ou liés au changement climatique. A l'échelle internationale, ces collections devraient faciliter la construction d'un réseau pour la conservation des ressources génétiques des caféiers, où pourront s'échanger matériel végétal et informations. Elles permettront aussi de mieux exploiter ces ressources dans les programmes de sélection dans le monde entier et ouvrent la voie aux études d'association et à la gestion globale de la diversité des caféiers.

Coffea canephora. Genetica, $142:$ 185-199.

Doi : 10.1007/s10709-014-9766-5

\section{PALMIER À HUILE \\ UN PAS VERS LA COMPRÉHENSION DE L'ANOMALIE FLORALE MANTLED}

estelle.jaligot@cirad.fr

[Diversité, adaptation et développement des plantes - UMR Diade]

L'anomalie florale mantled, qui touche les palmiers à huile issus de clonage in vitro, est d'origine épigénétique et se traduit par une malformation des étamines. Des chercheurs du Cirad, de I'IRD et du Felda ont tenté de comprendre comment le défaut de régulation épigénétique des palmiers anormaux agit sur l'un des principaux gènes contrôlant la mise en place des étamines. Ils ont détecté un transcrit alternatif produit par ce gène, plus court que le transcrit complet connu jusqu'alors, qui pourrait coder une protéine partiellement non fonctionnelle. En quantifiant le nombre de copies de chacun de ces deux transcrits dans les fleurs normales et mantled, les chercheurs ont montré que, dans ces dernières, la molécule courte est produite à un niveau suffisamment élevé pour entrer en compétition avec la forme longue. Cette découverte fournit une nouvelle piste pour expliquer, au niveau moléculaire, la formation des fleurs mantled et, à plus long terme, pour mettre au point un test de détection précoce de cette anomalie chez le palmier à huile.

PLoS One, 9 : e91896. Doi : 10.1371/journal.pone.0091896

\section{PARTENAIRES}

France $>$ Institut de recherche pour le développement (IRD). Malaisie > Felda Biotechnology Centre. 


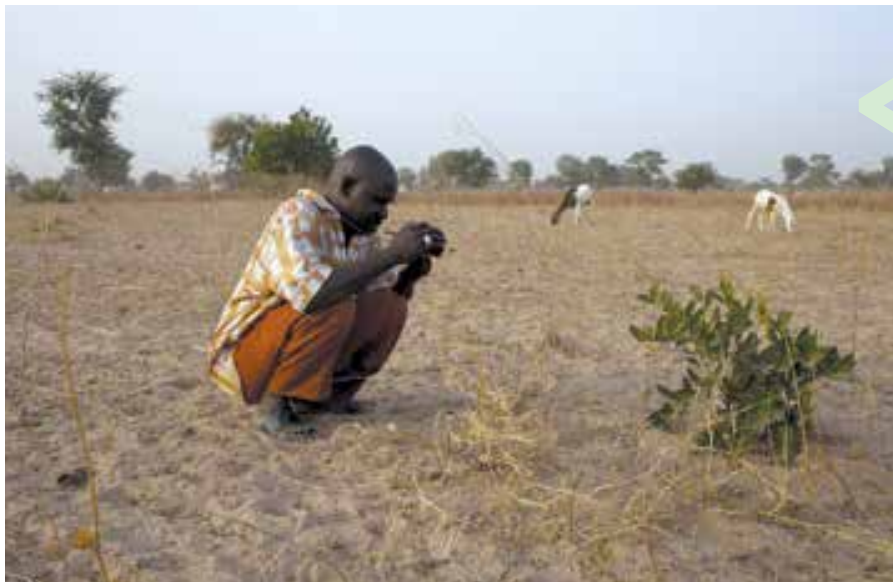

Démarche photo-ethnographique : un agriculteur photographie un indicateur pertinent selon lui pour caractériser un type de sol. @ B. Defives/Transit

\section{PARTENAIRES}

France > Centre de recherche et d'enseignement de géosciences de l'environnement (Cerege) ; Ecole centrale de Lyon ; École normale supérieure de Lyon ; Institut de recherche pour le développement (IRD, France) ;

Université Paris 7. Sénégal > Laboratoire commun de microbiologie

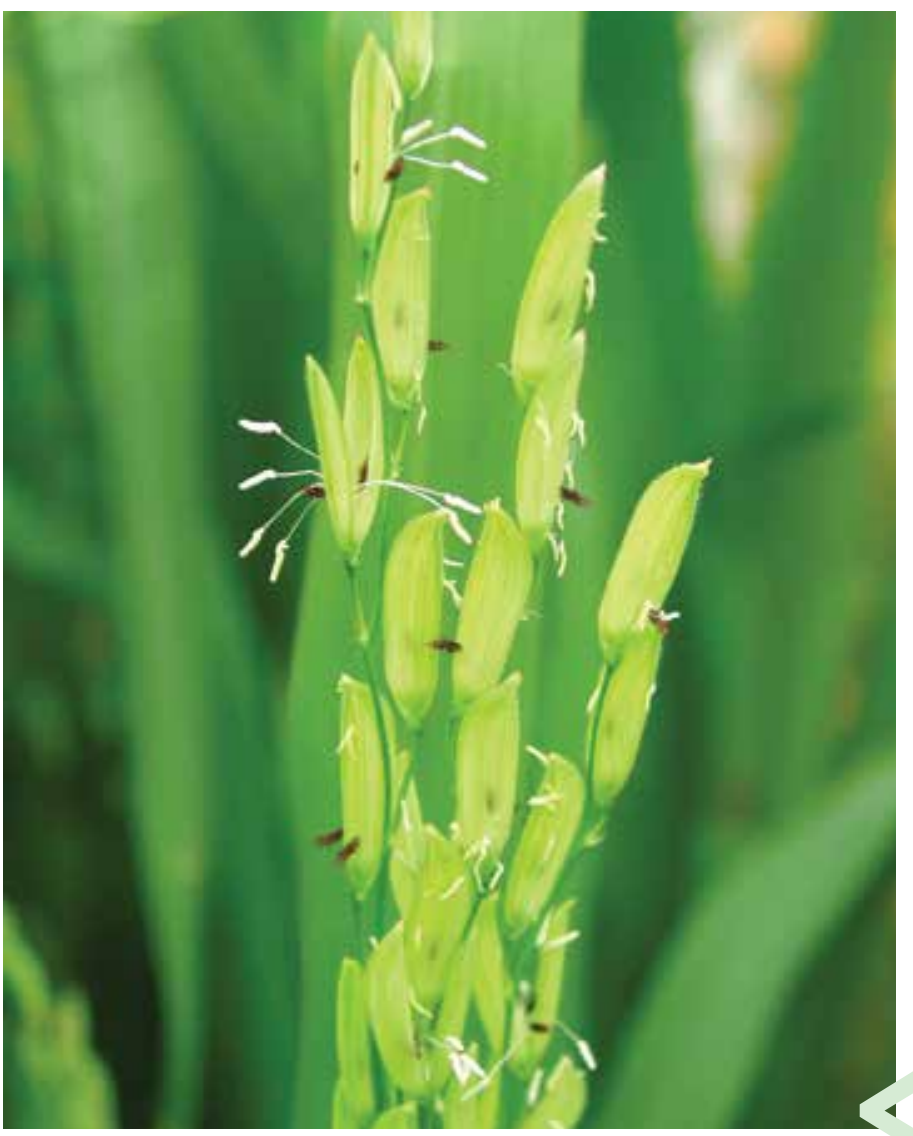

\section{RECHERCHE PARTICIPATIVE ENTRE SAVOIRS PAYSANS ET SAVOIRS SCIENTIFIQUES}

frederique.jankowski@cirad.fr

[Gestion des ressources renouvelables et environnement]

Les chercheurs ont choisi d'observer les processus de légitimation des savoirs locaux et scientifiques à l'œuvre au cours d'une recherche participative. Ils y ont exploré les modes de définition des savoirs et leur légitimité, ainsi que le lien entre légitimation et réappropriation des savoirs issus de l'action collective. Les processus de légitimation des savoirs renforcent ou affaiblissent les positions voire les appartenances des individus et des groupes au sein des communautés multiples dont ils sont membres. Au-delà des processus décrits, la démarche permet de dégager ce qu'est un espace des savoirs construits dans la recherche participative, un espace éclaté dont les frontières ne recouvrent pas celles des «laboratoires » au sens classique. Cet espace de production des savoirs participatifs met en lumière un paradoxe : le partenariat apporte une légitimation sociale aux chercheurs du projet, mais ne renforce pas nécessairement leur légitimité au sein de la communauté scientifique.

Natures, sciences, sociétés, 22 : 15-22. Doi : 10.1051/nss/2014015

\section{SÉLECTION DU RIZ IDENTIFICATION DU GÈNE DE STÉRILITÉ MÂLE MS-IR36}

nourollah.ahmadi@cirad.fr (Amélioration génétique et adaptation des plantes méditerranéennes et tropicales - UMR Agap]

Les sélectionneurs de riz du Cirad utilisent un gène de stérilité mâle pour faciliter les croisements dans leurs schémas d'amélioration. Ils ont identifié sa position dans le génome et sa fonction grâce aux ressources génomiques désormais bien développées pour cette culture. Ils ont mis en œuvre une démarche " classique » de clonage positionnel, qui consiste à isoler le gène à partir des marqueurs qui lui sont liés. Ils ont identifié très précisément le polymorphisme en cause : le SNP (single-nucleotide polymorphism, polymorphisme d'un seul nucléotide] associé à la stérilité mâle ms-IR36. Les chercheurs n'ont pas eu besoin de procéder à la validation fonctionnelle de ce gène, car, une fois identifié, ils ont découvert qu'il avait déjà été décrit à partir d'un autre mutant obtenu dans une variété japonica, même si le SNP en cause chez ce mutant était différent de celui lié à la stérilité mâle de ms-IR36.

Molecular Breeding, 33 : 555-567. Doi : 10.1007/s11032-013-9972-3

\section{PARTENAIRE}

France $>$ Institut national de la recherche agronomique (Inra).

Panicule d'une plante de riz mâle stérile au stade floraison : les anthères blanchâtres sont vides. @ N. Ahmadi/Cirad 


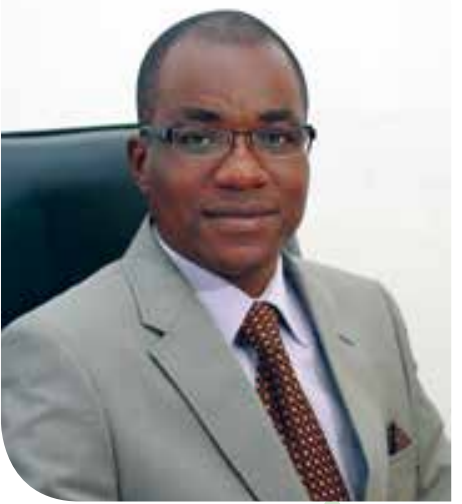

\section{DÉVELOPPER L'AGRICULTURE DURABLE ET PRÉSERVER LA FAUNE SAUVAGE}

Le Professeur Eddie Mwenje est président du Comité de pilotage du dispositif RP-PCP. II assure, avec les animateurs du DP, la coordination des actions des quatre organisations scientifiques de la plateforme et de l'ambassade de France.

edmwenje@gmail.com

www.rp-pcp.org

\section{POUVEZ-VOUS PRÉSENTER} LE DISPOSITIF RP-PCP ?

Eddie Mwenje : Le RP-PCP existe officiellement depuis 2007. Ce dispositif a été créé pour renforcer les capacités de recherche et les partenariats institutionnels au Zimbabwe et, plus largement, en Afrique australe en développant les collaborations, le partage et la diffusion de l'information avec les institutions de recherche française et européennes. L'objectif de ce dispositif à l'interface entre l'homme et la nature est de contribuer au développement durable, à la conservation et l'amélioration des niveaux de vie des populations rurales au Zimbabwe, un pays qui a connu de graves crises, mais aussi dans les nations frontalières. Nous sommes aujourd'hui face à de nombreux challenges dûs aux changements globaux et locaux qui menacent les populations rurales et la conservation de la biodiversité.

\section{QUELS SONT VOS DOMAINES}

\section{D'ACTIVITÉS ?}

E. M. : Le RP-PCP aborde quatre grandes thématiques : santé animale et environnement, incluant notamment la gestion des maladies infectieuses aux interfaces homme/ bétail/animaux sauvages; écologie fonctionnelle dans les zones d'interface; agriculture et conservation ; gouvernance et gestion durable des ressources naturelles. Plus de 70 chercheurs rattachés à des universités du Zimbabwe, d'Afrique du Sud, de Zambie, du Botswana, des institutions françaises et des organisations internationales, dont quatorze régulièrement impliqués dans la coordination de la plateforme, sont intervenus dans nos activités depuis 2007. Une grande part de

(c) Irène

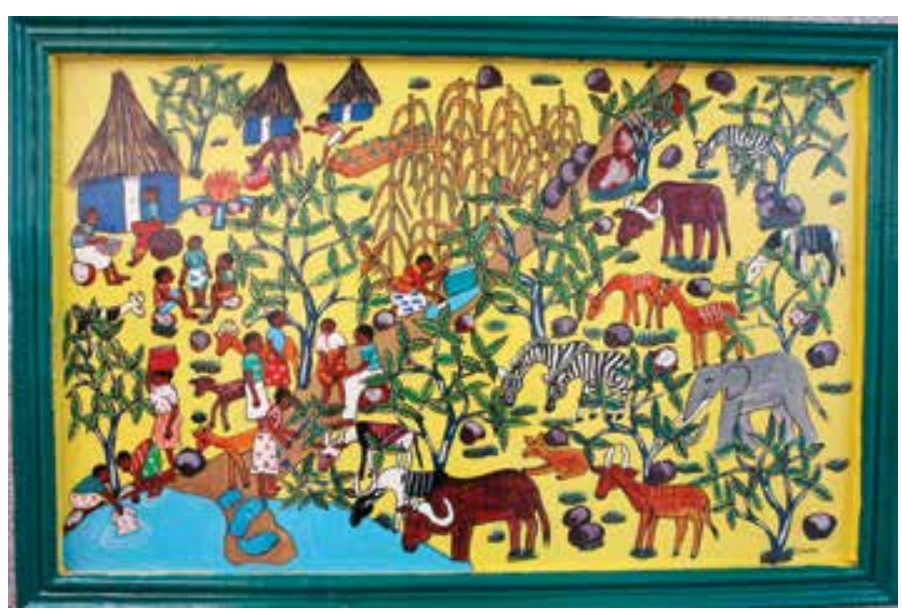

notre travail consiste également à former et encadrer des étudiants de troisième cycle, en majorité zimbabwéens et de la SADC.

\section{QUELLE EST LA VALEUR A JOUTÉE DU CIRAD?}

E. M. : Le Cirad est au Zimbabwe depuis le début des années 90 et les chercheurs ont une grande connaissance de la région. Nous profitons de leur expérience. C'est un partage qui va au-delà des aspects scientifiques, puisqu'il se fait aussi avec les politiques, les ONG et les communautés rurales. Les chercheurs du Cirad ont aussi un rôle fondamental dans la supervision de nos étudiants. Les compétences s'en trouvent renforcées pour les activités de recherche mais aussi pour

\section{PARTENAIRES}

France > CNRS : Institut de l'écologie et de l'environnement ; UMR Lyon1- LBBE ; UMR CEFE ; Cirad (UPR Agirs ; UPR Aïda ; UPR BSEF ; UPR Green; UMR Innovation ; UMR Moisa, UMR Intertryp ; UMR Innovation, UMR G-Eau).

Zimbabwe > University of Zimbabwe : Departments of Geography and Environmental Science; Animal Sciences, Biological Sciences ; Centre for Applied Social Science ; Faculty of Veterinary Science ; Soil science and agricultural engineering ; Agricultural economics ; National University of Science and Technology: Faculty of Forest Resources and Wildlife Management, Institute of Development Studies. 


\section{PÊCHE À SUMATRA \\ COMMENT S'ADAPTER \\ AU DÉCLIN DES RESSOURCES}

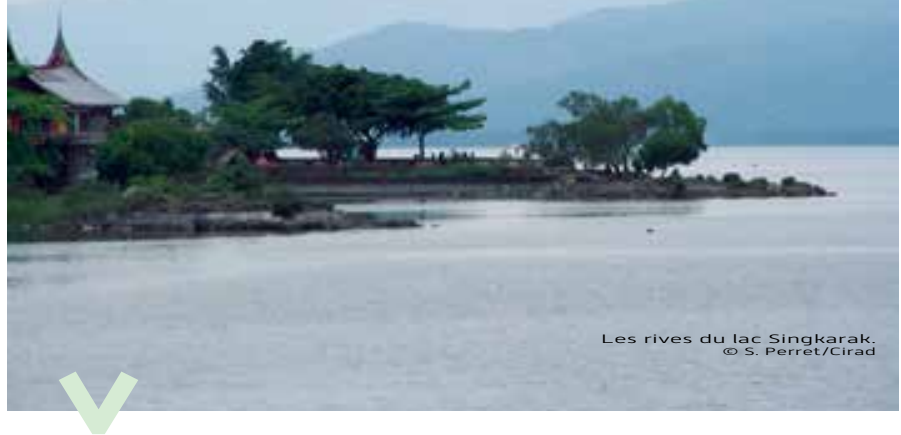

sylvain.perret@cirad.fr (Gestion de l'eau, acteurs et usages - UMR G-eau)

Dans les eaux du lac Singkarak, à Sumatra, les poissons se font plus rares et la pêche, une activité essentielle pour les populations riveraines, voit ses captures chuter. Quelles sont les causes de ce déclin, et comment y remédier? Une équipe pluridisciplinaire a mené une enquête de terrain pour comprendre les processus en jeu et trouver des solutions acceptables par tous les acteurs. Ses résultats ont été présentés aux autorités provinciales, aux communautés villageoises et aux représentants des pêcheurs. Ils ont permis de faire évoluer les perceptions de chacun. Les pêcheurs reconnaissent que certains d'entre eux pêchent trop par rapport aux ressources disponibles. Les communautés traditionnelles s'accordent sur le fait que les institutions en place doivent être révisées pour mieux exploiter les ressources du lac. Les autorités provinciales, quant à elles, se sont engagées à négocier avec les représentants des pêcheurs pour concevoir de nouvelles règles de pêche et lutter contre les excès.

Regional Environmental Change, $14: 1203-1214$

Doi : 10.1007/s10113-013-0554-z

PARTENAIRES. Indonésie > Université Andalas.

Thaïlande > Asian Institute of Technology (AIT)

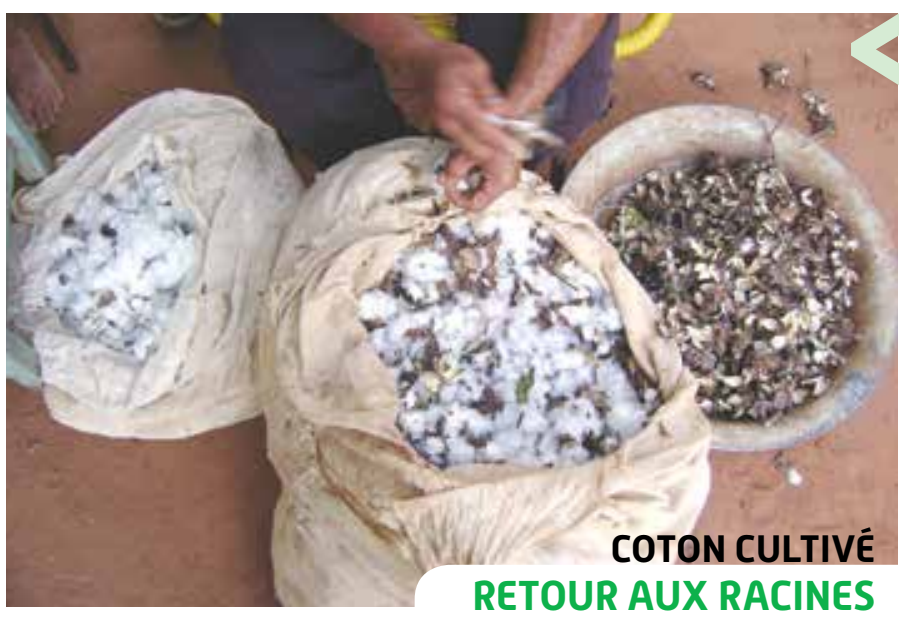

geo.coppens@cirad.fr

(Amélioration génétique et adaptation des plantes méditerranéennes et tropicales - Umr Agap]

\section{AGROSYSTĖME PTEROCARPUS OFFICINALIS/TARO SYMBIOTES ET TRANSFERT D'AZOTE}

antoine.galiana@cirad.fr (Laboratoire des symbioses tropicales et méditerranéennes - Umr Lstm)

La strate arborescente des forêts marécageuses de la région caraïbe (îles et continent) est dominée par Pterocarpus officinalis. Cette légumineuse est capable de fixer symbiotiquement l'azote atmosphérique et d'enrichir naturellement le sol en azote au profit des cultures sous-jacentes. En Guadeloupe, c'est sous ce couvert forestier qu'est conduite sans intrant la monoculture du taro (Colocasia esculenta). Pour comprendre le rôle des symbioses dans cet agrosystème traditionnel et durable, des chercheurs ont identifié les bactéries symbiotiques associées à $P$. officinalis. Si toutes appartiennent au genre Bradyrhizobium, elles se différencient nettement au niveau de l'espèce selon l'origine, insulaire ou continentale. De plus, ils ont montré que les associations entre provenances de $P$. officinalis et souches de Bradyrhizobium issues d'une même origine géographique étaient les plus efficientes en termes de nodulation, quantité d'azote fixé et production de biomasse. Par ailleurs, la capacité de Pterocarpus à transférer l'azote fixé au bénéfice du taro, par le biais des réseaux mycorhiziens partagés entre les deux espèces associées, est en cours d'étude sur le terrain.

Microbial Ecology, août 2014. Doi : 10.1007/s00248-014-0392-7

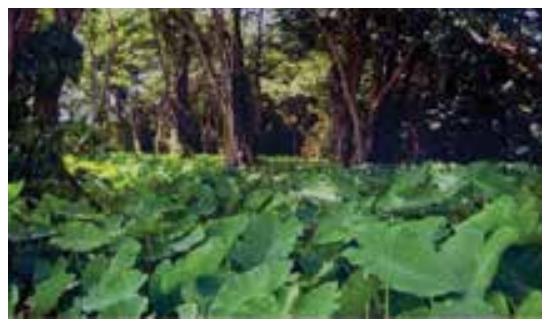

PARTENAIRES. France > Institut de recherche pour le développement (IRD) ; ministère de l'Ecologie, du Développement durable et de l'Energie ; Université des Antilles et de la Guyane.

Système agroforestier Pterocarpus officinalis/taro en forêt marécageuse, Guadeloupe. ๑) E. Saur, Université Antilles-Guyane (UAC)
La diversité génétique des ancêtres sauvages des plantes cultivées représente un potentiel important pour leur amélioration en réponse aux défis globaux. L'identification de caractères d'intérêt repose sur un travail préalable de caractérisation et de conservation. Dans le cas du cotonnier [Gossypium hirsutum], originaire de la Caraïbe et du golfe du Mexique, cette tâche est compliquée par l'existence de populations férales, dérivées de formes domestiquées anciennes, se maintenant dans des paysages anthropisés. En combinant approches écologique et génétique, les chercheurs ont montré que, malgré leur dispersion, les populations sauvages de cette région forment un ensemble homogène : inféodées à des environnements côtiers très arides, elles sont restées distinctes des populations férales, plus proches des cultivées. Les résultats confortent les hypothèses de leur dispersion par les courants marins, ainsi que de leur domestication au Yucatán, suivie d'une diffusion de la culture dans tout le Mexique. Ils permettront d'organiser l'indispensable conservation des formes sauvages et férales.

PLoS One, 9 : e107458 (19 p.]. Doi : 10.1371/journal.pone.0107458 


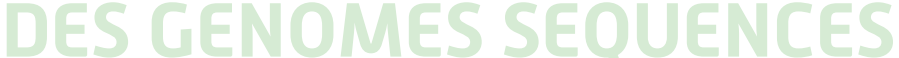

Tilapia > Les génomes de 5 espèces de Cichlidés, famille à laquelle appartient le tilapia, viennent d'être séquencés et comparés par le Cichlid Genome Consortium (CGC) dont le Cirad fait partie. Parmi les 27 laboratoires cosignataires de la publication dans la revue Nature, le Cirad, I'Université d'Aquaculture de Stirling (Grande-Bretagne) et l'Agricultural Research Organization (Israël) s'intéressent plus particulièrement aux retombées aquacoles du projet. Ils utiliseront la séquence de référence du tilapia pour identifier plus rapidement des séquences liées à des caractères d'intérêt aquacole et dans des programmes de sélection.

Nature, 18 septembre $2014.513: 375-381$

jean-francois.baroiller@cirad.fr (Intensification raisonnée et écologique pour une pisciculture durable - Umr Intrepid]

\section{4,3 millions de tonnes de tilapia produits chaque année dans plus de 100 pays}

Caféier > Une étude internationale a été coordonnée par des chercheurs du CEA (Genoscope), du Cirad, du CNRS, de l'IRD et de I'Université de Buffalo [USA]. Impliquant de nombreux laboratoires, elle a permis d'identifier, pour la première fois, une séquence génomique de référence pour les caféiers. Cette découverte revêt un double intérêt : fondamental, parce qu'elle améliore la compréhension de l'organisation du génome, de son fonctionnement et de son évolution ; et finalisé, parce qu'elle offre de nouvelles perspectives de sélection ou d'amélioration des variétés de caféier.

Science, 5 septembre 2014. Vol. 345 no. 6201 pp. 1181-1184. doi: 10.1126/science.1255274

benoit.bertrand@cirad.fr, Interactions Plantes Microorganismes Environnement [Umr IPME]

gaetan.droc@cirad.fr (Amélioration génétique et adaptation des plantes méditerranéennes et tropicales - Umr Agap)

PARTENAIRES. France > ANR ; Bioversity International ; CEA ; CNRS ; Inra ; IRD ; Montpellier SupAgro ; Nestlé R\&D ; UM2 ; Université d'Evry.

\section{2,25 milliards de tasses de café consommées chaque jour dans le monde}

Agrumes > Fédérés au sein du Consortium international de génomique des agrumes, des scientifiques du monde entier se sont associés pour décrypter le génome d'une dizaine d'agrumes et ainsi reconstituer l'histoire de leurs multiples croisements. Au départ, il a fallu établir la séquence génomique des neuf chromosomes d'un agrume. Le Cirad est à l'initiative du choix d'un clémentinier haploïde (un seul exemplaire de chaque chromosome] qui a permis de simplifier les analyses. Il a également coordonné* l'établissement de la carte génétique de référence.

Nature Biotechnology, 8 juin 2014. 32, 656-662 (2014). doi:10.1038/nbt.2906

patrick.ollitrault@cirad.fr (Amélioration génétique et adapatation des plantes méditérranéennes et tropicales - Umr Agap]

*Dans le cadre du projet Citrusseq, financé par l'ANR et conduit en partenariat entre le Genoscope (CEA), le Cirad, l'Inra et l'Ivia.

\section{PRODUCTION DU CAOUTCHOUC NATUREL L'ÉTHYLĖNE, UNE HORMONE ESSENTIELLE}

pascal.montoro@cirad.fr

(Amélioration génétique et a<daptation des plantes méditerranéennes et tropicales - Umr Agap]

La production de caoutchouc naturel est sous le contrôle intime de l'éthylène. Apporté de manière exogène (éthéphon) ou synthétisé par les tissus de l'écorce, cette hormone végétale agit sur différents processus cellulaires. Dans les cellules à latex, l'éthylène favorise l'écoulement du latex après saignée et la reconstitution du cytoplasme des cellules endommagées. Les facteurs de réponse à l'éthylène (ERFs) sont les derniers facteurs de transcription de la voie de signalisation de l'éthylène. Ces ERFs induisent l'expression des gènes dépendant de l'éthylène (transporteurs, enzymes, protéines de défense, etc.). Une analyse fine du transcriptome de l'hévéa dans différents tissus a établi le rôle primordial de l'éthylène dans le métabolisme des laticifères. Ces cellules possèdent aussi des fonctions exacerbées comme le contrôle de la réponse à l'hypoxie. Cela permettrait la mise en place de la fermentation pour l'utilisation des sucres sous faible teneur en oxygène dans les tissus de l'écorce afin de maintenir la biosynthèse des chaînes de cis-1,4 polyisoprène sous conditions de stress. Ces connaissances ouvrent des perspectives sur le développement de marqueurs fonctionnels pour la génétique et de nouveaux types de stimulant de la production.

PLoS One. 2014. 9(6):e99367). Doi : 10.1371/journal.pone.0099367

\section{PARTENAIRES}

Belgique > Société Financière des Caoutchoucs [SOCFINCO]. Chine > Beijing Institute of Genomics, Chinese Academy for Tropical Agricultural Sciences. France > Institut Français du Caoutchouc; Michelin ; Société internationale de plantation d'hévéas (SIPH]. Indonésie > Rubber Research Institute of Indonesia. Thaïlande > Rubber Research Institute of Thailand.

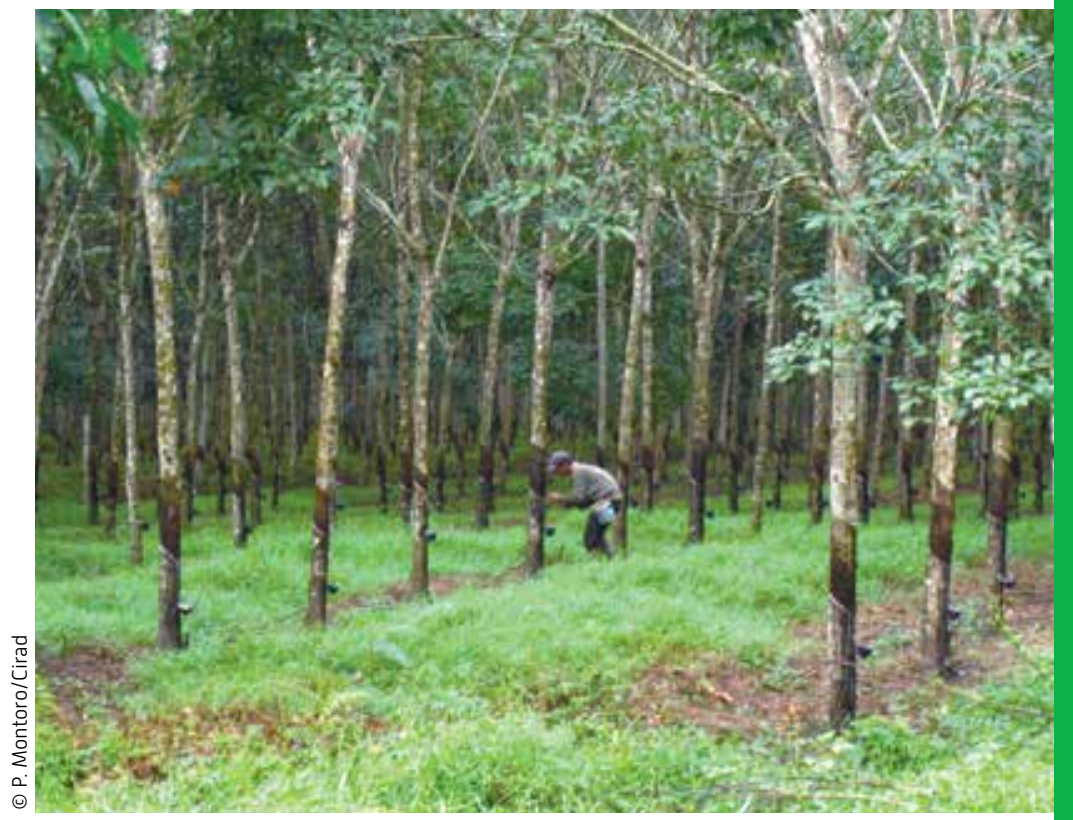




\section{CLIMAT ET PERTES VARIÉTALES DE MÉMOIRE D'AGRICULTEURS}

christian.leclerc@cirad.fr

[Amélioration génétique et adaptation des plantes méditerranéennes et tropicales - UMR Agap]

En recourant à la mémoire de 208 agriculteurs kenyans, il a été possible de reconstituer l'évolution de leur système de culture qui a favorisé le maïs au détriment du sorgho et du mil $(\boldsymbol{A})$, et de mesurer l'effet des variations climatiques sur la perte de variétés pour huit espèces vivrières majeures, entre 1961 et 2006. La comparaison de ces données aux relevés météorologiques permet d'apprécier la fiabilité de la mémoire paysanne, particulièrement pour les situations climatiques extrêmes (défaut ou excès de pluie), et d'étudier rétrospectivement le lien entre climat et pertes variétales. Ainsi, la probabilité de perdre une variété augmente fortement lorsque le nombre de jours de pluie est inférieur à 28 ou supérieur à 40 , et la précipitation totale inférieure à 400 mm ou supérieure à 750 mm (B). Enfin, une analyse par espèce explique comment la dynamique qui favorise le maïs accroit le risque de perte de semences, rendant les systèmes de culture d'Afrique de l'Est plus vulnérables aux variations climatiques.

Weather, Climate and Society. 2014. 6[3]: 354-370.

PARTENAIRES. France > Agence nationale de la recherche (ANR), projet Picrevat ; Centre européen de recherche et d'enseignement en géosciences de l'environnement (Cerege) ; Centre de recherches de climatologie (CRC) ; Laboratoire d'océanographie et du climat : expérimentations et approches numériques (Locean)/ Université de Bourgogne ; Montpellier SupAgro ; Institut de recherche pour le développement (IRD).

Analyse rétrospective des savoirs paysans: A. Dynamique agricole (1961-2006) ; B. Perte de variétés et précipitation.
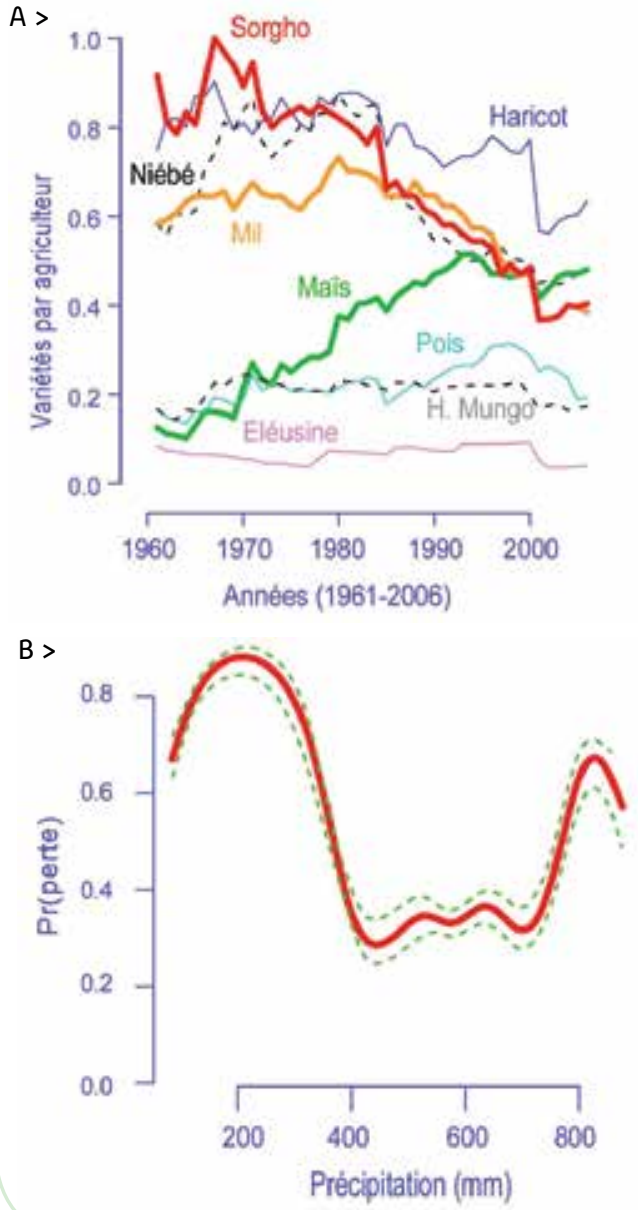

\section{CANNE À SUCRE}

\section{ESTIMER LES RENDEMENTS DES PETITS PRODUCTEURS PAR TÉLÉDÉTECTION}

julien.morel@cirad.fr (Agroécologie et intensification durable des cultures annuelles - UPR Aïda)

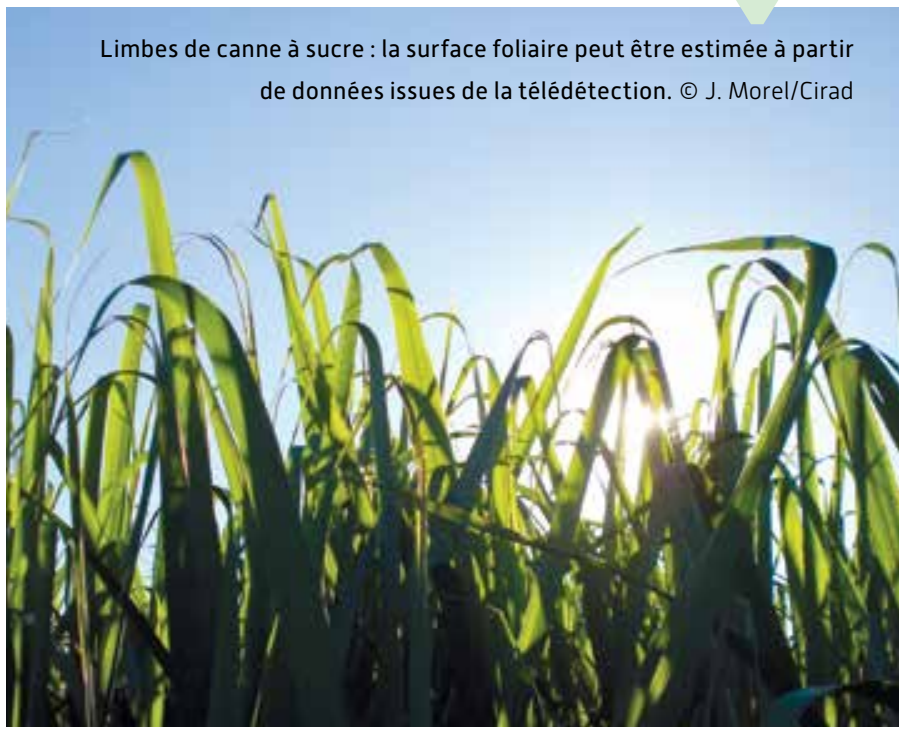

Dans les petits pays producteurs de canne à sucre, l'hétérogénéité des pratiques culturales et la multitude des parcelles sont la règle. Diffıcile, dans ces conditions, d'estimer la production, qui pourtant est une condition essentielle à la pérennisation des filières sucrières. En comparant plusieurs méthodes d'estimation de cette production basées sur les indices de végétation fournis par la télédétection, les chercheurs ont constaté que la méthode empirique était la plus précise et la recommandent dans le contexte de l'agriculture familiale. Avec cette méthode, il est cependant indispensable de disposer d'un nombre suffisant d'images satellitaires, pour décrire correctement l'évolution de l'indice de végétation au cours du temps. L'arrivée des satellites Sentinel-2, qui fournissent des images en accès libre d'une résolution spatiale de 10 mètres avec une fréquence d'acquisition de 5 jours, devrait lever cette contrainte.

Remote Sensing, 6 : 6620-6635. Doi : 10.3390/rs6076620

PARTENAIRES. France > Institut de recherche pour le développement (IRD) : Syndicat du sucre de la Réunion. 


\title{
AGROSYSTĖMES PRODUCTIFS ET ÉCOLOGIQUES EN AFRIQUE DE L'OUEST
}

\author{
Dans les zones de savanes d'Afrique de l'Ouest, la population est fortement \\ dépendante de l'agriculture mais les crises économiques, alimentaires et \\ climatiques appauvrissent les paysans. Le dispositif de recherche en partenariat \\ Asap* s'est fédéré autour de l'intensification écologique avec pour objectif \\ l'amélioration de la productivité d'agrosystèmes familiaux durables associant \\ polyculture et pastoralisme.
}

\section{POUVEZ-VOUS PRÉSENTER \\ LE DISPOSITIF ASAP \\ EN QUELQUES MOTS ?}

Souleymane Ouedraogo: Alors que ce dispositif de dimension régionale basé au Burkina Faso a formellement été créé en 2010, une communauté de scientifiques intéressés par les systèmes de production agricoles travaillait depuis plusieurs années autour de nombreux projets initiés par le Cirad, notamment le projet DURAS (Promotion du développement durable dans les systèmes de recherche agricole du
Sud]. Asap se focalise aujourd'hui sur les questions d'intensification écologique : comment concevoir des agrosystèmes familiaux durables en Afrique de l'Ouest? Le Burkina Faso, le Mali, le Sénégal, le Niger et, depuis peu, la Côte d'Ivoire, sont associés à nos programmes scientifiques dans le cadre d'Asap.

\section{QUELS SONT LES ENJEUX?}

S. 0. : L'enjeu prioritaire vise à accroître la productivité afın d'assurer aux paysans la sécurité alimentaire et des revenus suffisants.
Concevoir une agriculture plus productive passe nécessairement par la prise en compte de l'environnement ce qui a conduit Asap à se fédérer autour du processus d'intensification écologique. Nous travaillons sur trois échelles : la parcelle, l'exploitation et le territoire. Nos stratégies s'appuient sur la diminution de l'utilisation des intrants de synthèse, une meilleure intégration agriculture et élevage et enfin le respect des savoirs locaux dans la conception d'innovations en collaboration avec les paysans.

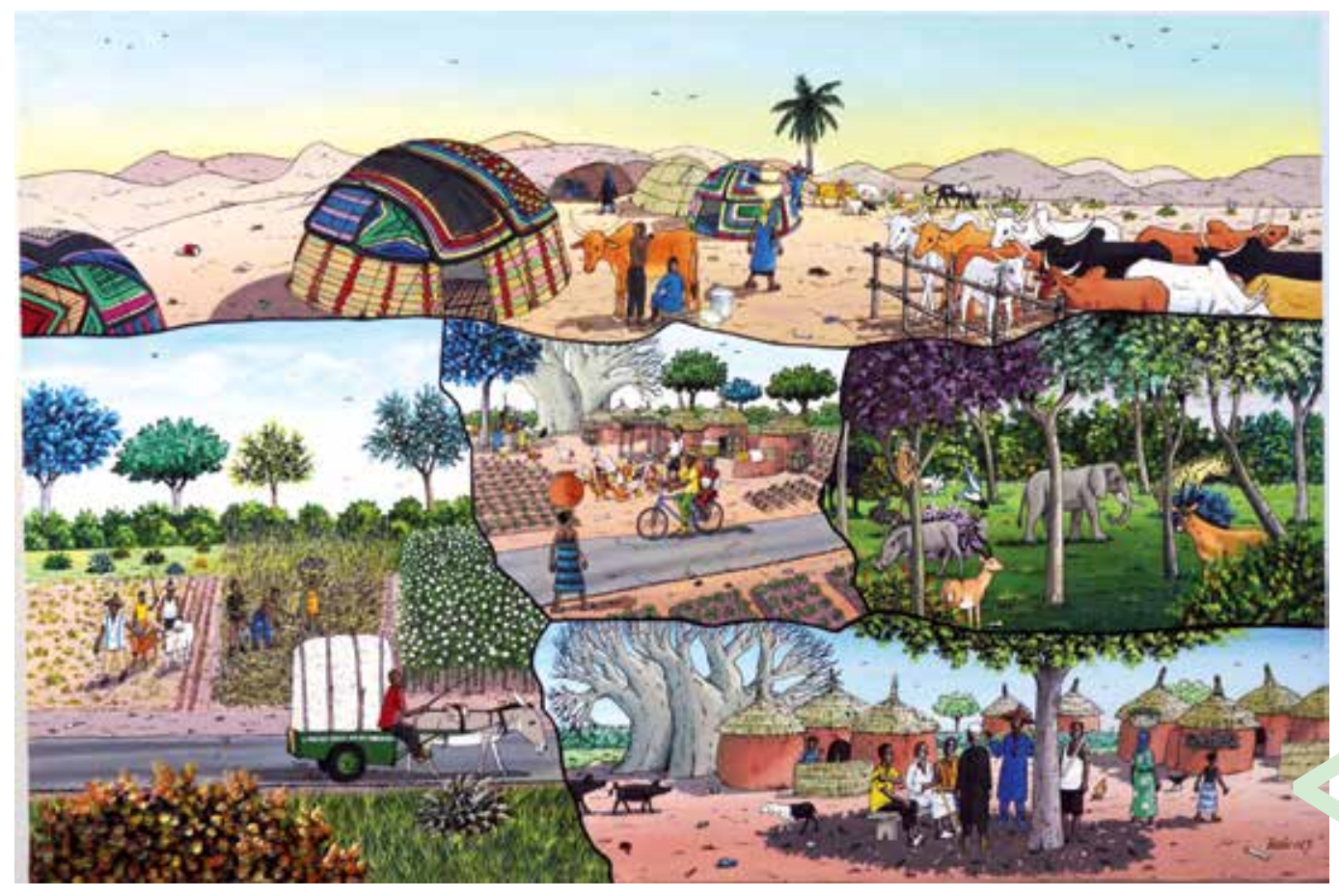

QUELLE EST LA VALEUR AJOUTÉE DU CIRAD POUR LE DISPOSITIF ?

S. O. : Nous avons une longue expérience de coopération avec le Cirad. Notre partenariat a permis de réunir des scientifiques travaillant dans un champ d'action commun ce qui est très fédérateur. Nous pouvons interagir entre chercheurs de différentes disciplines, et c'est une force. Cette plateforme scientifique fournit un cadre idéal pour la formation des étudiants. Asap nous apporte une visibilité vis-à-vis des autres institutions, nous permet d'attirer d'autres acteurs, d'obtenir plus facilement des ressources et de créer de nouvelles passerelles.

* Asap : Systèmes agro-sylvo-pastoraux en Afrique de l'Ouest

\section{PARTENAIRES}

Burkina Faso > Centre International de Recherche-Développement surl'élevage en zone subhumide (Cirdes) ; Institut de l'environnement et de recherches agricoles (Inera) ; Institut d'économie rurale (IER); Université Polytechnique de Bobo-Dioulasso : Institut de Développement Rural (UPB/IDR). France $>$ Cirad (UMR Innovation ; UMR Selmet; UPR Aïda ; UPR BSEF ; UMR Agap).

Tableau réalisé pour le DP Asap c) Mahamoudou Zinkone 


\section{VALORISATION DE LA BIOMASSE}

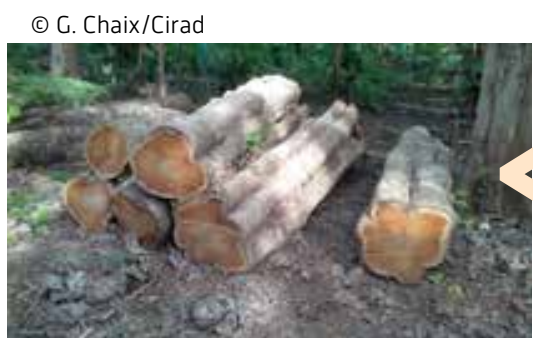

Le bois de teck est apprécié pour ses propriétés exceptionnelles, en particulier sa résistance aux parasites. Les chercheurs ont mis au point une technique rapide d'analyse du bois et de prévision de sa durabilité à partir du dosage des quinones qu'il renferme. Chez le teck, la durabilité naturelle résulte en effet de la présence de molécules appartenant à la famille des quinones, synthétisées au cours de la formation du bois de cœur.

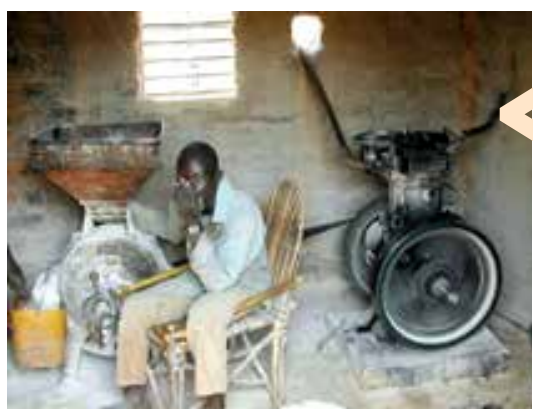

La motorisation est un facteur de développement rural. () D. Litvine

Le Burkina Faso s'est lancé en 2007 dans la production d'huile de pourghère, un biocarburant qui peut remplacer le gazole en milieu rural. Produit, transformé et consommé sur un même territoire, ce biocarburant a la capacité de s'intégrer dans les modes de vie des populations, stimule la demande énergétique, mais modifie aussi l'opinion des populations à son égard. Ce sont les résultats d'une enquête de terrain menée par une équipe du Cirad pour comprendre com-

\section{DURABILITÉ NATURELLE DU TECK UNE TECHNIQUE RAPIDE DE PRÉVISION}

nadine.amusant@cirad.fr (Ecologie des forêts de Guyane - UMR Ecofog)

La teneur de ces quinones dans le bois, déterminée par spectroscopie proche infrarouge en réflexion diffuse (Spir), permet donc de prédire sa durabilité. Cette évaluation, indirecte et rapide, de la durabilité naturelle des bois de teck peut être réalisée à partir de poudre de bois. Utilisée dans le cadre de l'amélioration de la qualité de service des bois auprès des acteurs de la filière, elle permettra d'exploiter et d'utiliser ces bois de manière plus rationnelle. Avec pour résultats une gestion durable des exploitations forestières et un matériel végétal sélectionné sur des critères de qualité.

Journal of Near Infrared Spectroscopy, $22: 35-43$. Doi : 10.1255 jnirs.1091

PARTENAIRES. Côte d'Ivoire > Institut national polytechnique Félix Houphouët Boigny; Société de développement forestier [Sodefor]. Malaisie > Yayasan Sabah Group.

\section{AGROCARBURANTS AU BURKINA FASO LES CIRCUITS DE PROXIMITÉ STIMULENT LA DEMANDE}

marie-helene.dabat@cirad.fr

(Acteurs, ressources et territoires dans le développement - UMR Art-Dev)

laurent.gazull@cirad.fr

[Biens et services des écosystèmes forestiers tropicaux - UPR BSEF]

ment la proximité de l'offre influe sur la demande, et comment cette demande se construit et s'exprime. La demande est généralement étudiée à partir de facteurs techniques et économiques. Elle pourrait l'être aussi sur des critères sociaux, subjectifs et contextuels, qui permettent de comprendre comment se construisent et s'ajustent les préférences des consommateurs par rapport à l'offre énergétique. Une approche originale, qui a des retombées pratiques en matière de recommandations aux porteurs de projets et aux décideurs, mais aussi de stratégie d'offre énergétique qui soit à la fois viable sur le plan technique et génératrice de développement local.

Ecological Economics, 100 : 85-95 Doi : 10.1016/j.ecolecon.2014.01.018

\section{PARTENAIRES}

\section{Burkina Faso > Institut}

international d'ingénierie de l'eau et de l'environnement [2iE].

France > Université de Montpellier

\section{Biocombustibles}

Projet Catapult *

La pyrolyse flash est un procédé de conversion thermochimique de la biomasse conduisant à de hauts rendements en bio-huiles. Ces biocombustibles liquides ouvrent de nouvelles perspectives de valorisation couplée matière/énergie de type bioraffinerie thermochimique. L'objectif du projet Catapult * est d'améliorer la qualité des bio-huiles, par l'utilisation de catalyseurs durant la pyrolyse, et d'évaluer la pertinence d'une co-valorisation en molécules bio-sourcées et carburants alternatifs. Ce projet ANR répond à des enjeux aussi bien scientifiques qu'industriels en couvrant des domaines de compétences tels que l'optimisation de catalyseurs, le développement de procédés intégrés et les analyses/tests de qualité des produits

francois.broust@cirad.fr (Biomasse, bois, énergie, bioproduits - UPR BioWooEB]

PARTENAIRES. France $>$ Agence Nationale de la Recherche (ANR] ; Céramiques Techniques Industrielles (CTI) ; Institut de Recherche sur la Catalyse et l'Environnement (Ircelyon-Cnrs) ; Société financière de la filière française des oléo-protéagineux (Sofiproteol).

*Catapult: Catalyse en pyrolyse pour une co-valorisation de biohuiles en chimie et carburants] 


\title{
ALIMENTATION DURABLE
}

\section{Aliments-mangeurs MOINS DE SENS, PLUS DE DISTANCE}

\author{
Des chercheurs étudient l'évolution \\ de la consommation alimentaire au Vietnam. \\ Le rôle des sens, notamment, modifie la façon \\ d'acheter. La modernisation des systèmes \\ alimentaires créé une distance entre l'aliment \\ et le mangeur qui peut s'avérer anxiogène.
}

Entretien avec Muriel Figuié, sociologue au Cirad.

Comment les sens sont-ils mobilisés dans l'acte d'achat?

Muriel Figuié. Dans les systèmes alimentaires traditionnels comme les marchés, le corps est engagé dans la sélection des produits alimentaires par ses cinq sens: l'odorat et la vue permettent d'écarter les produits altérés voire malsains, le toucher d'évaluer la fermeté, l'ouïe d'échanger des informations avec le vendeur, et bien sûr le goût de tester. Par exemple, au Vietnam les poulets s'achètent souvent vivants. L'animal est inspecté, l'allure de sa crête, la température de ses pattes, la propreté de son cloaque... Cela renseigne sur sa qualité gustative mais aussi sanitaire. Dans les supermarchés, qui se développent rapidement depuis une vingtaine d'années au Vietnam, c'est une autre histoire. Les grands magasins sont le domaine de la réfrigération, de la chaîne du froid. Quand il y en $a$, les informations se lisent sur l'emballage qui empêche le touché, l'odorat et le goût d'être mis à contribution. II faut connaître les marques, savoir interpréter une liste d'ingrédients, une date limite de consommation (DLC).

\section{Comment les consommateurs} vietnamiens se sont-ils adaptés à l'achat en supermarchés ?

M. F. Au début, il était courant de trouver les sachets de légumes éventrés par les clients désireux de les sentir, de les toucher. Autre exemple significatif, une cliente qui, à la lecture de la DLC portée sur un emballage de viande de porc, s'est exclamée: " je ne veux pas qu'on me dise jusqu'à quand je peux le manger, je veux savoir quand le porc a été abattu, et je déciderai moi-même ! ". La DLC est une notion nouvelle dans ce pays où les achats de produits frais sont généralement quotidiens. Elle symbolise un transfert d'expertise et de pouvoir de décision du client vers le vendeur. Cela alors même que la confiance envers les producteurs est mise à mal par plusieurs crises alimentaires (lait contaminé, grippe aviaire, etc.].
Muriel Figuié [à droite), en quête de sens au Vietnam, avec Paule Moustier, directrice de I'UMR Moisa.

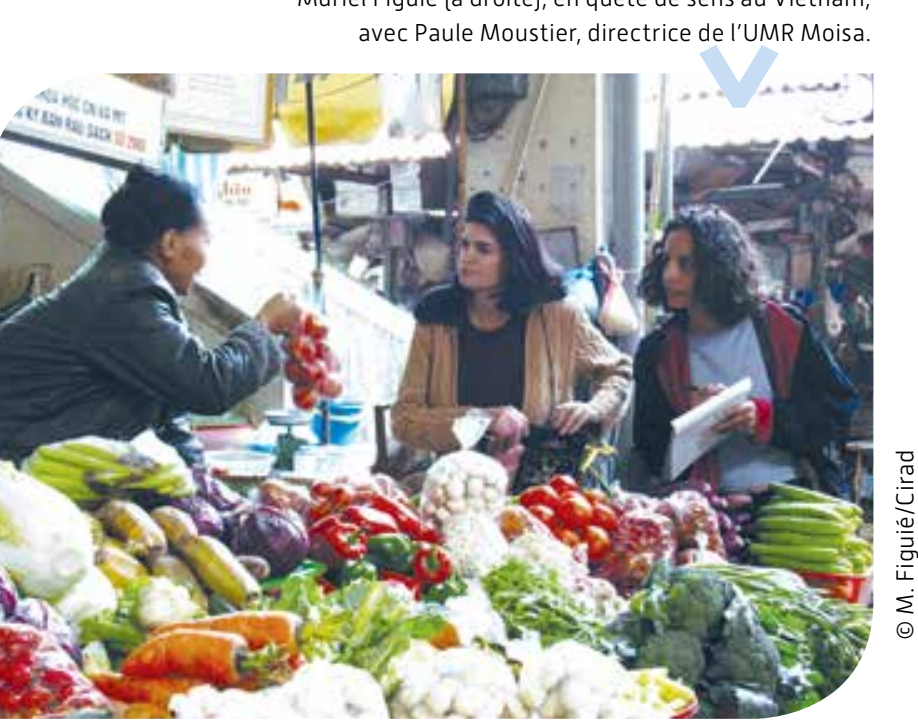

Selon vous, la modernisation des systèmes alimentaires créer une distance entre I'aliment et le mangeur. Expliquez-nous..

M. F. Effectivement, il s'agit d'une distance physique entre les lieux de production et de consommation et par l'emballage des produits, d'une distance cognitive car les aliments résultent de processus industriels de plus en plus complexes et mal connu des consommateurs, et enfin d'une distance sociale car les aliments sont plus difficilement associables à des personnes familières (producteur, commerçant, etc.). Nous défendons la thèse que cette distanciation entre le mangeur et I'aliment est troublante et anxiogène. Elle accentue probablement les inquiétudes des consommateurs sur la sécurité sanitaire des produits. Ces craintes font que, au Vietnam, les consommateurs se tournent vers des produits importés. Notre équipe étudie comment les producteurs locaux peuvent se réapproprier le marché et bénéficier ainsi du boom de la demande urbaine en produits alimentaires, principalement issu de la classe moyenne émergente. Ces recherches s'inscrivent dans le projet du dispositif de recherche et d'enseignement en partenariat Malica (Marchés et agricultures pour les villes en Asie].

muriel.figuie@cirad.fr (Marchés organisation institutions et stratégie d'acteurs - UMR Moisa]

Figuié M., Bricas N. 2014. Faire ses courses au Vietnam aujourd'hui : quand les supermarchés touchent aux sens. Anthropology of Food: $12 \mathrm{p}$. http://aof.revues.org/7445

\section{Avec la modernité alimentaire, les consommateurs} doivent reconstruire les bases de la confiance dans leur alimentation : renoncer à une expertise sensorielle directe pour se fier à des signes indirects (marque, label, tampon vétérinaire, ...). 


\section{MARAIICHAGE URBAIN AU VIETNAM LA QUALITÉ SANITAIRE PASSE PAR LE CONSEIL TECHNIQUE}

paule.moustier@cirad.fr

(Marchés, organisations, institutions et stratégies d'acteurs - UMR Moisa]

L'action collective des producteurs peut jouer un rôle essentiel dans la qualité des produits alimentaires. Son efficacité a été étudiée chez les maraîchers de la région de Hanoi, au Vietnam, par les chercheurs du Cirad, de l'Inra et leurs partenaires. Leurs conclusions rejoignent celles d'autres travaux : la formation technique fournie à leurs membres par les associations de producteurs est déterminante pour garantir la qualité sanitaire des aliments. Sur les soixante groupes testés, dix présentaient un niveau de toxicité supérieur aux doses autorisées pour au moins l'un de leurs échantillons. C'est le conseil technique dispensé par les producteurs les plus expérimentés aux membres du groupe qui influe le plus sur la qualité sanitaire des produits. L'environnement institutionnel et économique n'a pas d'impact significatif, alors que l'éducation joue en faveur d'une utilisation croissante de produits chimiques. Tant que les producteurs n'ont pas atteint un certain niveau de connaissances dans l'utilisation des produits chimiques, l'efficacité des mesures incitatives, qu'elles concernent les débouchés spécifiques, le contrôle ou la labellisation, reste limitée.

Journal of Development Studies, 50 : 715-730. Doi : 10.1080/00220388.2013.874555
C'est le conseil technique qui influe le plus sur la qualité sanitaire des produits.

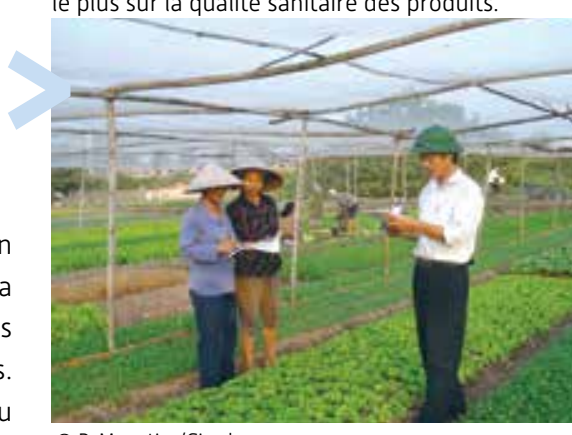

(c) P. Moustier/Cirad

PARTENAIRES. France $>$ Institut national de la recherche agronomique (Inra) Royaume-Uni > Natural Resources Institute (NRI). Vietnam > Fruit and Vegetable Research Institute [Favri] ; Vietnam Academy of Agricultural Sciences (Vaas).

\title{
RIZ CUIT
}

\section{UN MODĖLE POUR PRÉDIRE SA TEXTURE}

\author{
aurelien.briffaz@cirad.fr christian.mestres@cirad.fr \\ (Démarche intégrée pour l'obtention d'aliments de qualité - UMR Qualisud]
}

Le mode de cuisson du riz détermine sa saveur et surtout sa consistance, deux caractéristiques recherchées par les consommateurs. D'où l'idée de modéliser les transferts et les réactions qui se produisent au cours de sa cuisson pour mieux prévoir et contrôler sa texture. Une équipe du Cirad a conçu un modèle de cuisson du riz, qui assimile le grain à une sphère et couple transferts d'eau, déformation et gélatinisation de l'amidon. L'originalité de ces travaux réside dans le fait qu'ils mettent directement en relation les distributions simulées de propriétés (teneur en eau, gélatinisation) au sein du grain et les données de texture. D'un point de vue pratique, I'utilisation du modèle s'avère très prometteuse pour la conception de cuiseurs à riz innovants, qui pourraient proposer des programmes de cuisson adaptés à la texture, plus ou moins ferme ou collante, recherchée par les différents consommateurs.

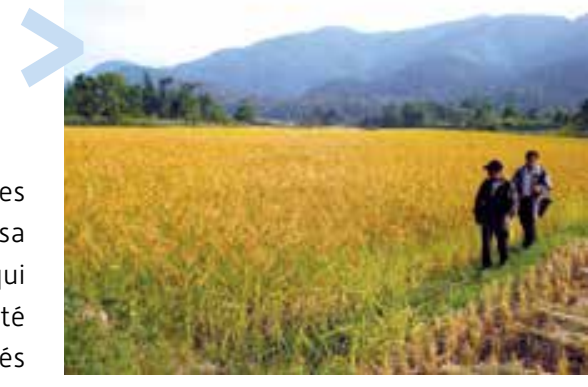

Champ de riz au Laos. @ D. Sautier/Cirad

PARTENAIRE. France > Montpellier SuPAgro.

Journal of Food Engineering, 141 : 99-106. Doi : 10.1016/j.jfoodeng.2014.05.008

\section{BANANE PLANTAIN APTITUDE À LA CUISSON ET QUALITÉ NUTRITIONNELLE A}

olivier.gibert@cirad.fr

[Démarche intégrée pour l'obtention d'aliments de qualité - UMR Qualisud]

La banane plantain, un aliment de base en Afrique et en Amérique latine, est consommée cuite et présente à maturité une forte teneur en amidon. Une équipe du Cirad s'intéresse aux propriétés technologiques de ces bananes, qui déterminent leur aptitude à la transformation et leurs usages, afın d'optimiser leur qualité nutritionnelle lorsqu'elles sont cuites. Les chercheurs ont mis en évidence que, même s'il est gélatinisé à $100 \%$, l'amidon de plantain au stade vert n'est pas totalement digestible et que la température est le facteur déterminant pour piloter la conversion de l'amidon et sa digestibilité. Ils ont aussi mis en évidence d'importantes différences dans l'évolution de la fermeté entre les génotypes, lors de la cuisson. Ces différences, imputables pour une grande part au degré de gélatinisation de l'amidon, permettent d'identifier les génotypes qui auront la préférence des consommateurs. Les chercheurs se sont également intéressés à l'évolution de la qualité nutritionnelle de la banane plantain lors de sa cuisson à l'eau. Pour ce faire, ils ont construit un diagramme de phase qui prédit la digestibilité de son amidon en fonction de son degré de gélatinisation, de la température de traitement et de la teneur en eau du produit.
Diversité des plantains de la collection du Carbap, le Centre africain de recherches sur bananiers et plantains, au Cameroun. O. Gibert @ Cirad 


\section{SANTÉ DES ANIMAUX ET DES PLANTES}

\section{Lutte intégrée contre les insectes \\ "IL EST INDISPENSABLE DE CONSIDÉRER \\ L'ENSEMBLE DU SYSTĖME »}

Deux entomologistes, Jérémy Bouyer, vétérinaire, et Thierry Brévault, agronome, s'associent pour affirmer d'une même voix les conditions d'une lutte intégrée efficace contre les insectes. Une gestion intelligente des ravageurs nécessite de prendre en compte la globalité du système : des processus biologiques et écologiques aux logiques de décisions des acteurs du territoire concerné.

Vous avez récemment cosigné un article et pourtant vous ne travaillez pas ensemble. Quel était le propos?

Thierry Brévault. Jérémy est vétérinaire et moi agronome. Mais nos recherches se recoupent dans la lutte contre les insectes nuisibles des cultures ou vecteurs de maladies. Avec cet article, nous voulions affirmer que la lutte intégrée contre les ravageurs ne peut être efficace qu'à condition de considérer le système dans sa globalité. Cela implique de délimiter une aire fonctionnelle (population du ravageur ou bassin de production), de mieux connaître les processus écologiques qui participent à la régulation des populations du ravageur ciblé sur cette aire, mais aussi les acteurs concernés et leurs logiques de décision.

La connaissance des processus écologiques qui régulent les populations de ravageurs est selon vous un passage obligé. Donneznous un exemple...

Jérémy Bouyer. Nous venons de participer avec succès à l'éradication des mouches tsé-tsé dans une partie de la région des Niayes, au Sénégal. Ces glossines sont le vecteur de la trypanosomose animale, une maladie considérée comme le principal obstacle au développement de l'élevage en Afrique subsaharienne. La campagne, fondée sur la lutte intégrée, a mêlé piégeage de mouches, traitements insecticides du bétail et lâchers aériens de glossines mâles stériles dans sa phase d'éradication. Pour une lutte efficace, il a fallu au préalable délimiter la population de mouches et bien connaître ses dynamiques écologiques. Après trois ans d'études de ces processus, nous avons pu agir de manière ciblée sur les habitats privilégiés des glossines. Ces connaissances ont permis à la fois une réduction des coûts et une parfaite efficacité de la campagne d'éradication.

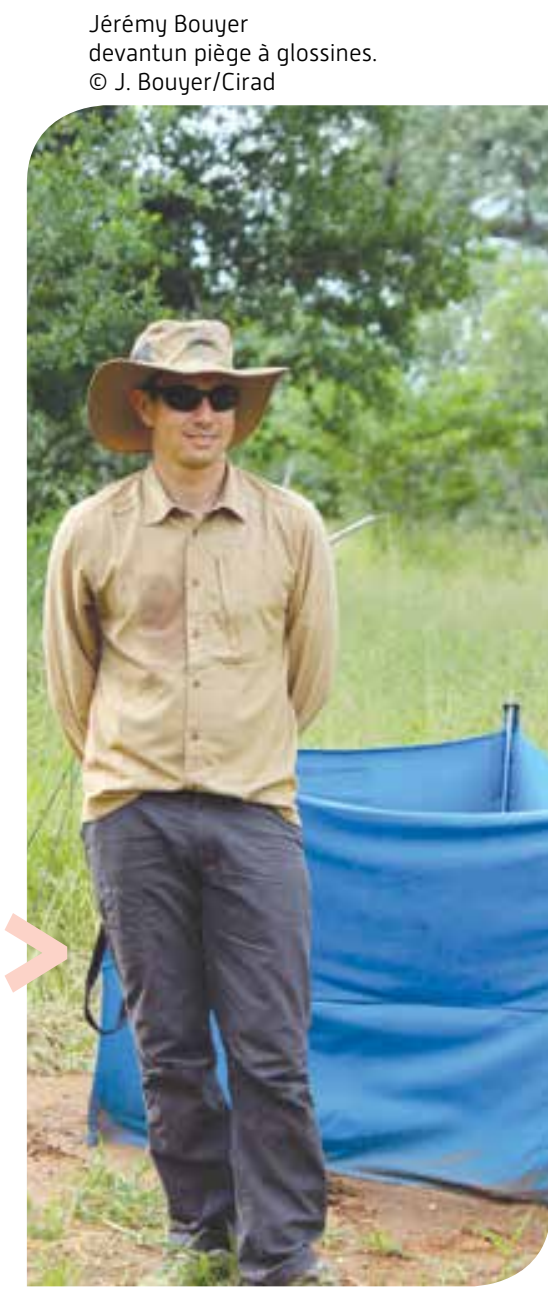

\section{$14 \%$ de la production agricole est perdue avant et après récolte}

\section{à cause des ravageurs ! La lutte intégrée est la seule méthode d'avenir}

à l'heure où les pesticides sont dangereux pour la santé et l'environnement

et sont de moins en moins efficaces. 
Thierry Brévault. D. R.

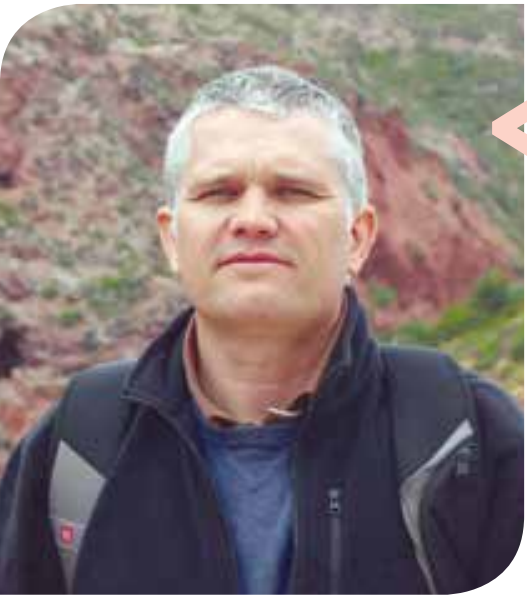

Outlooks on Pest Management, 25 (3) : 212-213. Doi : 10.1564/v25_jun_05

Proceedings of the National Academy of Sciences of the United States of America, 111: 10149-10154.

Doi : 10.1073/pnas.1407773111
Pourquoi est-il également indispensable de tenir compte des acteurs du système pour lutter contre des ravageurs?

T. B. Car en se plaçant à une échelle plus large que la parcelle cultivée, on s'aperçoit très vite que les insectes se déplacent sans frontière, plusieurs acteurs sont donc concernés. Si un agriculteur dispose de techniques simples pour contrôler un ravageur donné dans son champ, par exemple par l'application d'insecticides, une ré-infestation est à craindre si aucune mesure de lutte n'est mise en œuvre dans les champs voisins. De même, si certains agriculteurs traitent leurs champs avec des insecticides à large spectre, ils vont à l'encontre de la conservation des ennemis naturels comme agents de régulation des populations du ravageur ciblé. Il faut alors considérer le socio-écosystème. D'où la nécessité d'actions menées collectivement et donc d'une solide coordination. La mise en œuvre des stratégies de lutte intégrée implique bien souvent une recherche de consensus, la concertation est donc indispensable.

J. B. En outre, la réalité du terrain nous montre que la lutte intégrée est souvent mal exploitée : considérée comme une simple boîte à outils et utilisée localement. Nous préconisons un changement fondamental d'approche pour une meilleure gestion préventive et effective des ravageurs. Pour cela, il est indispensable d'impliquer les acteurs du territoire, de former les agriculteurs pour leur transmettre aussi bien les connaissances des dynamiques écologiques des ravageurs que la nécessité de l'action collective concertée.

\section{jeremy.bouyer@cirad.fr}

[Contrôle des maladies animales exotiques et émergentes - UMR CMAEE]

\section{thierry.brevault@cirad.fr}

(Agroécologie et Intensification Durable des cultures Annuelles - UPR Aida)
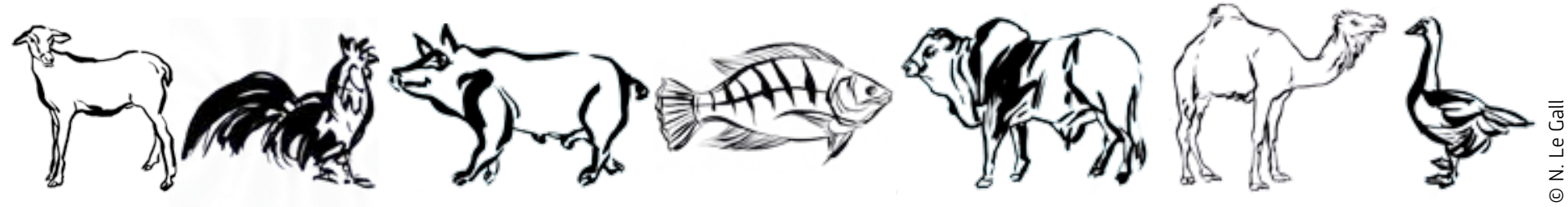

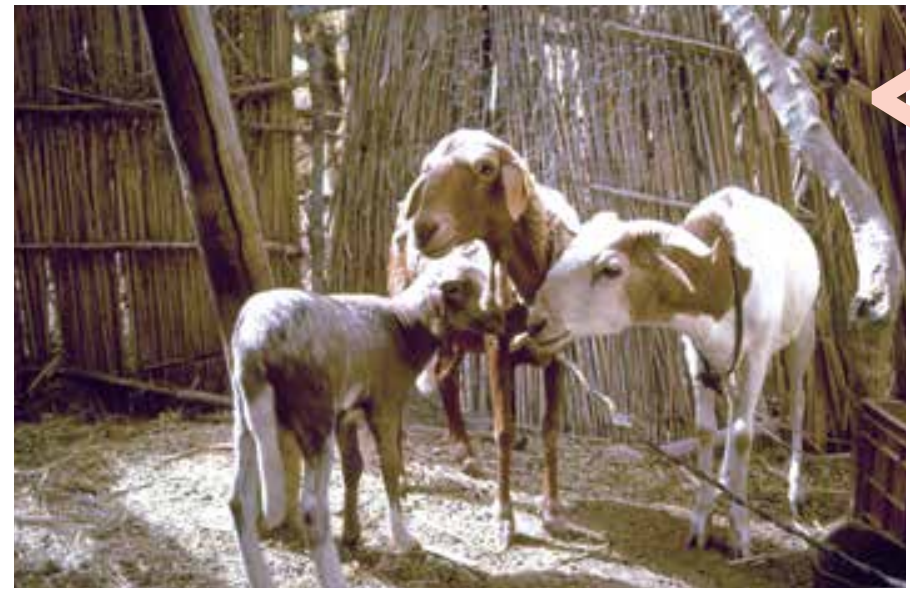

Elevage de moutons au Sénégal. @ R. Lancelot/Cirad

Vaccine, 32 : 3772-3779. Doi : 10.1016/j.vaccine.2014.05.025

\section{PARTENAIRES}

Autriche $>$ Animal Production and Health Laboratory

Ethiopie $>$ National Animal Health Diagnosis and Investigation Center (Nahdic)

France $>$ Institut national de la recherche agronomique (Inra)

\section{VARIOLES ET PESTE DES PETITS RUMINANTS UN VACCIN BIVALENT À L'ÉPREUVE DE L'IMMUNITÉ ACQUISE}

philippe.caufour@cirad.fr

[Contrôle des maladies animales exotiques et émergentes - UMR CMAEE]

En Afrique, au Moyen-Orient et en Asie, les varioles et la peste des petits ruminants font des ravages dans les troupeaux de chèvres et de moutons. Un vaccin bivalent, efficace à la fois contre les varioles et la peste, a été développé. Cependant, comme c'est le cas avec d'autres vecteurs vaccinaux, son efficacité peut être limitée par l'immunité acquise contre l'une de ces maladies dans les régions où elles sont endémiques. Pour tester cette hypothèse, les virologues ont mené une série d'expériences sur des chèvres préalablement immunisées pour l'un ou l'autre des virus. Ils ont ainsi démontré que, si le vaccin recombinant protégeait efficacement des animaux préalablement infectés par le virus PPR contre une infection par le virus CaPV, il n'en allait pas de même concernant la protection contre la PPR d'animaux préalablement immunisés contre le CaPV : la protection vaccinale contre le virus PPR chutait nettement chez ces animaux. A l'instar de ce que l'on observe avec les principales familles de vecteurs viraux, la préimmunité, dirigée contre le vecteur viral CaPV, entraîne donc une perte d'efficacité lors de l'utilisation des recombinants CaPV sur les terrains où ces virus sont installés et circulent de façon endémique. 


\section{CONFLIT OU SYNERGIE ? COMBATTRE L'ÉROSION ET RÉGULER LES BIOAGRESSEURS DU MIL}

alain.ratnadass@cirad.fr

(Fonctionnement agroécologique et performances des systèmes

de culture horticoles - UPR Hortsys]

Au Sahel, l'érosion des sols est un problème majeur. Sur les sols sableux, cette érosion due au vent peut aussi endommager les champs de mil, principale culture vivrière de la région. Pour limiter ces effets néfastes et maintenir la fertilité des sols, on peut laisser les tiges de mil dans les champs après la récolte. Mais quel est l'impact de ces pratiques sur la survie des ravageurs du mil ? Les chercheurs ont étudié ces effets dans deux localités du Niger. Ils ont constaté que la coupe des tiges de mil à la récolte et leur stockage sur plateforme offraient les conditions les plus favorables à la survie des larves du foreur des tiges. En revanche, cette survie est plus faible si on couche les tiges au sol. Dans le cas des ravageurs des panicules, la gestion des résidus de récolte pour réduire l'érosion et maintenir la fertilité du sol ne permet pas de réguler les populations de bioagresseurs. Pour le foreur des tiges, elle entre même en conflit avec la lutte contre le ravageur puisqu'elle autorise la survie de ses larves.

PARTENAIRES. Niger > Centre de multiplication des semences de Doukoudoukou; Institut international de recherche sur les cultures des zones tropicales semi-arides (Icrisat) ; Institut national de recherches agronomiques du Niger (Inran) ; Université Abdou Moumouni.

Agriculture Ecosystems and Environment, 186 : 144-147. Doi : 10.1016/j.agee.2014.01.021

Cette étude a été réalisée dans le cadre du projet Hope for dryland cereal de la fondation Bill et Melinda Gates.

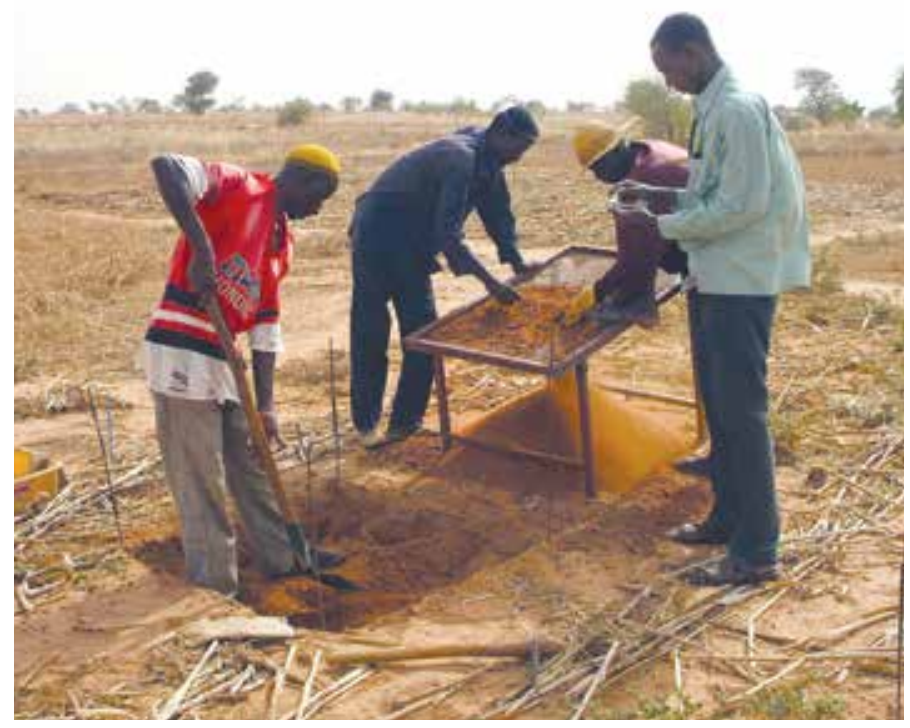

Tamisage d'échantillons de sol pour déterminer la macrofaune tellurique des parcelles de mil. ๑ A. Ratnadass/Cirad

\section{CULTURES FRUITIĖRES À LA RÉUNION DE NOUVEAUX PIĖGES POUR LES MOUCHES DES FRUITS}

serge.quilici@cirad.fr

(Peuplements végétaux et bioagresseurs

en milieu tropical - UMR PVBMT]
Une femelle de la mouche des fruits Ceratitis rosa (c) D. Vincenot/Chambre d'Agriculture de la Réunion
A la Réunion, les chercheurs ont mis au point un système de piégeage de masse pour deux espèces de mouches des fruits, Ceratitis rosa et $C$. capitata, particulièrement dévastatrices. Le système, qui combine un piège, un attractif pour femelles et une quantité minime d'insecticide, est aussi efficace que les méthodes classiques de lutte sans en présenter les inconvénients. II requiert, en effet, une quantité extrêmement réduite d'insecticide et ce, uniquement dans les pièges sans aucune application de produit sur les fruits. II est facile à utiliser : une fois les pièges installés, l'attractif et l'insecticide restent actifs pendant toute la durée de sensibilité des fruits. Il permet de s'affranchir des traitements par taches répétés à base de spinosad, qui risquent d'entraîner, à moyen terme, l'apparition d'une résistance chez les mouches. C'est un système idéal pour protéger les vergers d'agrumes de moyenne et de haute altitude.

PARTENAIRES. Espagne > Institut de Recerca i Tecnologia Agroalimentàries (Irta). France > Association réunionnaise pour la modernisation de l'économie fruitière, légumière et horticole (Armeflhor).

Pest Management Science, 70 : 448-453. Doi : 10.1002/ps.3591

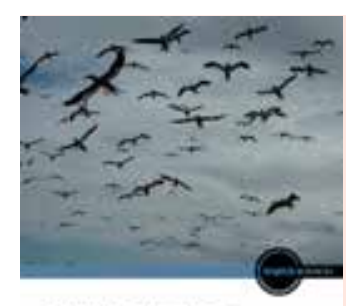

FAUNE SAUVAGE, BIODIVERSTEE ET SANTE, QUEIS DEFIS?

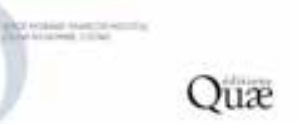

\section{Faune sauvage, biodiversité et santé, quels défis ?}

Aujourd'hui, la biodiversité s'érode cent fois plus vite que naturellement. Parallèlement, les maladies émergentes ou ré-émergentes sont en pleine expansion. Existerait-il des liens entre biodiversité et transmission des pathogènes ? Les pertes de biodiversité s'accompagnent-elles d'une augmentation des risques sanitaires infectieux? La résistance aux antibiotiques en est-elle en partie responsable? La faune sauvage est-elle devenue, malgré elle, sentinelle de la qualité sanitaire de l'environnement? Cet ouvrage aide à mieux comprendre les interactions entre les êtres vivants et leur environnement. Et aussi comment la recherche trouve des alternatives grâce à une nouvelle approche de l'écologie de la santé pour bien-être de tous.

serge.morand@cirad.fr (Animal et Gestion Intégrée des Risques - UPR Agirs) Faune sauvage, biodiversité et santé, quels défis ? Coordination éditoriale de Serge Morand, François Moutou, Céline Richomme. Ed. Quae, 2014 CRC Press, août 2014 


\section{ECHAUDURE DES FEUILLES DE LA CANNE À SUCRE}

\section{LA VARIÉTÉ A UN RÔLE DÉTERMINANT DANS LA CONTAMINATION}

jean-heinrich.daugrois@cirad.fr (Biologie et génétique des interactions plante-parasite - UMR BGPI]

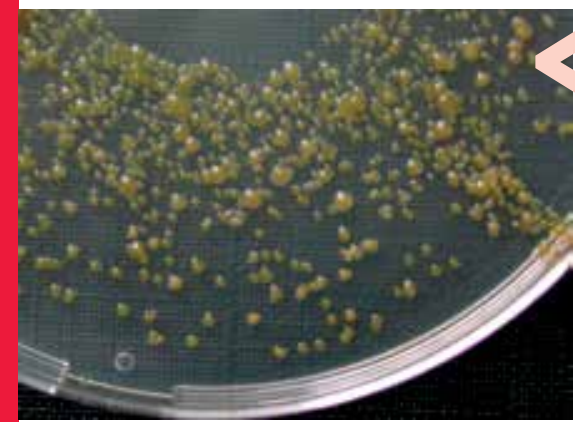

Xanthomonas albilineans issu de gouttes sur un milieu de culture. ๔ J.H. Daugrois/Cirada

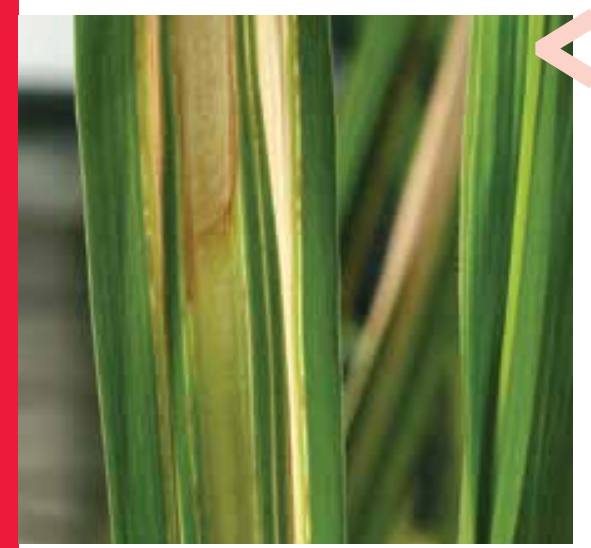

Lignes blanches et nécroses causées par Xanthomonas albilineans sur une feuille de canne à sucre. ๑ P. Rott/Cirad
A la Guadeloupe, la bactérie Xanthomonas albilineans, responsable de l'échaudure des feuilles de la canne à sucre, se transmet par voie aérienne et colonise la surface foliaire avant de se propager à l'intérieur des tissus. Une équipe du Cirad a démontré que la variété avait une influence déterminante sur cette phase précoce de l'infection et sur l'évolution ultérieure de la maladie. Les chercheurs ont ainsi constaté que, d'une variété à l'autre, la densité des populations présentes sur les feuilles peut fortement varier, selon la pluviométrie du cycle de récolte, de même que les symptômes foliaires. Chez certaines variétés de canne, des densités élevées de populations épiphytes entraînent des symptômes foliaires sévères ; chez d'autres, ces mêmes densités n'induisent que très peu voire aucun symptôme. Il existe donc différents mécanismes de résistance, qui jouent un rôle dans la multiplication de la bactérie à la surface ou dans la feuille, ou un mécanisme qui module le passage des populations entre l'extérieur et l'intérieur de la feuille.

Plant Disease, 98 : 191-196. Doi : 10.1094/PDIS-02-13-0195-RE

\section{UN NOUVEAU MÉCANISME D'INFECTION DÉCOUVERT}

philippe.rott@cirad.fr (Biologie et génétique des interactions plantes-parasites - UMR BCPI)

On pensait que la bactérie pathogène Xanthomonas albilineans se multipliait exclusivement dans le système vasculaire de la canne à sucre. Des chercheurs du Cirad et de l'IRD ont démontré qu'elle est aussi capable d'envahir d'autres tissus des feuilles et de la tige. Dans la feuille, elle est présente non seulement dans le xylème, mais aussi dans le phloème (le tissu conducteur de la sève élaborée), les cellules du parenchyme vasculaire et non vasculaire, ainsi que dans les cellules de l'épiderme. Dans la tige, la bactérie est observée dans les cellules du parenchyme vasculaire et dans le phloème. Plus surprenant, la bactérie est aussi détectée dans les cellules du parenchyme non vasculaire de la tige (appelées aussi cellules de stockage) et dans les espaces intercellulaires de ces cellules. C'est la première fois qu'une telle caractéristique est décrite chez une bactérie phytopathogène. Une découverte qui ouvre de nouvelles perspectives sur les stratégies d'invasion bactériennes.

PARTENAIRE. France > Institut de recherche pour le développement (IRD)

Open Biology, 4 [130116] : 13 p. Doi : 10.1098/rsob.130116

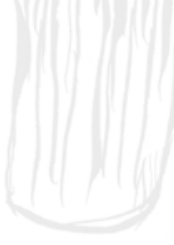

\section{RIZ PLUVIAL À MADAGASCAR \\ L'AGRICULTURE DE CONSERVATION POUR LIMITER LA PYRICULARIOSE}

mathilde.sester@cirad.fr [Agroécologie et intensification durable des cultures annuelles - UPR Aida]

Dans la région des Hautes-Terres de Madagascar, le riz pluvial est souvent victime de la pyriculariose, une maladie fongique dévastatrice pour les cultures. L'agriculture de conservation, qui est pratiquée pour limiter l'érosion dans cette région de collines, a-t-elle une influence sur le développement de la maladie ? Les chercheurs du Cirad et du Fofifa ont réalisé pendant cinq ans des essais sur plusieurs variétés de riz pluvial. Leurs résultats sont mitigés: si l'agriculture de conservation réduit bien l'incidence de la maladie, elle entraîne aussi des retards de croissance, qui pénalisent les rendements surtout pour les variétés les plus sensibles. Les chercheurs explorent à présent de nouvelles pistes pour améliorer l'implantation de la culture tout en préservant les effets bénéfiques de ces systèmes sur la tolérance aux maladies.

PARTENAIRE. Madagascar > Centre national de la recherche appliquée au développement rural [Fofifa] Plant Pathology, 63 : 373-381. Doi : 10.1111/ppa.12099

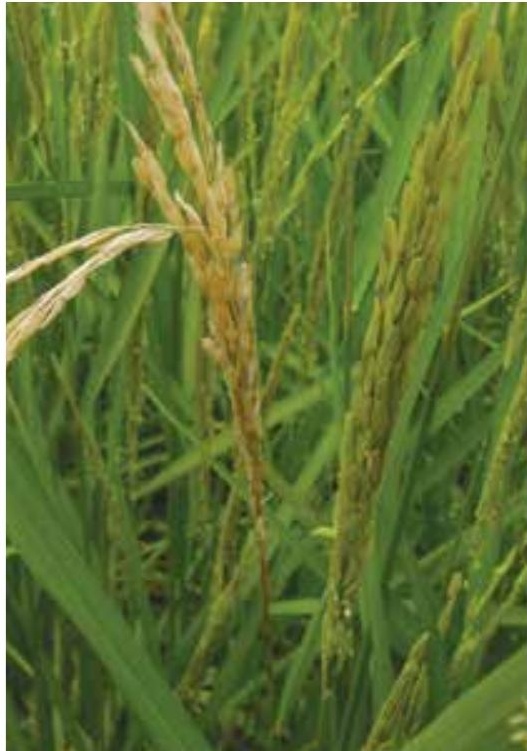




\section{IGNAME AFRICAINE \\ DES SÉQUENCES DE BADNAVIRUS DÉTECTÉES DANS SON GÉNOME}

denis.filloux@cirad.fr [Biologie et génétique des interactions plante-parasite - UMR BGPI]

Le Cirad et I'Inra, en partenariat avec le NRI et I'IITA, ont révélé la présence de séquences virales de badnavirus intégrées au génome des ignames africaines du complexe Dioscorea cayenensis-rotundata. C'est une première chez ces plantes à tubercules largement cultivées en Afrique de l'Ouest. Plusieurs de ces séquences, baptisées eDBV pour endogenous Dioscorea bacilliform virus, ont été partiellement caractérisées sur le plan moléculaire. Leur structure, particulièrement complexe, comprend des séquences fortement réarrangées, répétées ou inversées et fait actuellement l'objet d'une étude approfondie. Les analyses réalisées notamment à partir de matériel végétal issu de graines ont aussi permis d'identifier plusieurs espèces d'ignames porteuses de ces séquences. Les badnavirus infectent de nombreuses plantes tropicales et provoquent des maladies parfois graves. La question est maintenant de savoir si certaines des séquences endogènes découvertes chez les ignames pourraient, comme chez le bananier, provoquer des infections spontanées.

PARTENAIRES. rance $>$ Institut national de la recherche agronomique (Inra).

Nigeria > International Institute of Tropical Agriculture (IITA). Royaume-Uni > Natural Resources Institute (NRI).

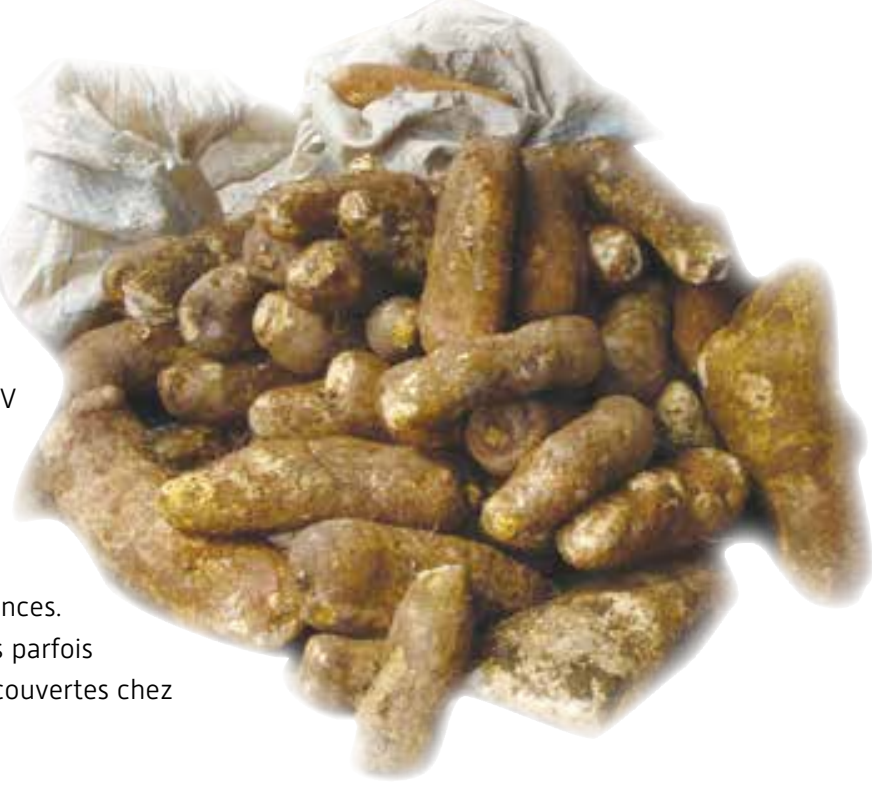

Molecular Plant Pathology, 15 : 790-801. Doi : 10.1111/mpp.12137

Les ignames sont des éléments essentiels de l'alimentation des populations des régions tropicales, en particulier en Afrique de l'Ouest. (c) D. Filloux/Cirad

\section{Lancement du réseau PACSUN \\ Le manioc sous surveillance}

L'alimentation d'environ 700 millions d'Africains dépend du manioc, de plus en plus central notamment suite à la flambée des prix des aliments de base. Mais deux viroses menacent dangereusement le tubercule : la mosaïque du manioc, présente partout en Afrique, qui réduit fortement les rendements et la striure brune du manioc qui le rend inconsommable. Pour combattre ces maladies, 28 institutions internationales se sont associées pour créer le réseau pan-africain de surveillance du manioc, PACSUN. Sa priorité : suivre la progression de la striure brune pour mettre en place des mesures limitant sa propagation.

Un site international de contrôle et d'assainissement des boutures de manioc devrait aussi voir le jour à La Réunion, rare zone indemne de ces deux viroses.

* Pan-African Cassava Surveillance Network

jean-michel.lett@cirad.fr

(Peuplements Végétaux et Bioagresseurs en Milieu Tropical - UMR PVMT)

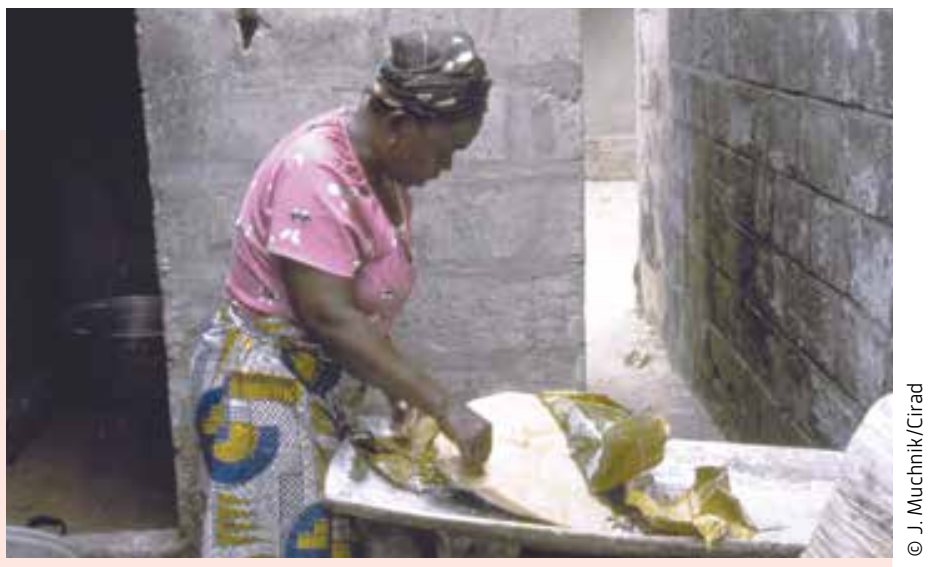

\section{L'alimentation d'environ 700 millions d'Africains} dépend du manioc.

Le lancement du réseau PACSUN et d'un site international de transit du manioc au Pôle de Protection des Plantes à La Réunion font suite à l'atelier international intitulé « Surveillance et contrôle des maladies du manioc en Afrique « organisé à La Réunion, du 10 au 13 juin dernier par le Cirad et l'IRD, sous l'égide du GCP21 (Global Cassava Partnership for the $21^{\text {st }}$ Century). 


\section{CHANCRE ASIATIQUE DES AGRUMES MIEUX CONNAITRE LA BACTÉRIE RESPONSABLE}

olivier.pruvost@cirad.fr

(Peuplements végétaux et bioagresseurs en milieu tropical - UMR PVBMT)

Le chancre asiatique, causé par la bactérie Xanthomonas citri pathovar citri, représente une contrainte sanitaire majeure de l'agrumiculture en régions tropicales et subtropicales et une menace pour les pays agrumicoles du Bassin méditerranéen. La génétique des populations de $X$. citri est peu connue, notamment dans son aire d'origine, l'Asie. Pourtant, cette zone est la source de futures souches émergentes. Un schéma de génotypage pour l'épidémio-surveillance de cette bactérie a été developpé. Ce schéma, ciblant des séquences minisatellites, est facile à mettre en œuvre techniquement et les données peuvent être partagées en ligne sur un site dédié. Il a permis d'identifier quatre lignées génétiques majeures pour le pathovar citri en relation avec leur gamme d'hôtes au sein des Rutacées, famille botanique à laquelle appartiennent les agrumes. Une seule de ces lignées est quasiment responsable de l'expansion géographique hors d'Asie pendant la première moitié du XXe siècle. La caractérisation, en Iran et en Ethiopie, de souches jusqu'alors inconnues montre son intérêt pour la surveillance. Ce schéma est actuellement appliqué à la compréhension de la réémergence de cette bactérie en Afrique subsahélienne.

PLOS ONE. Doi : 10.1371/journal.pone.0098129

http://www.biopred.net/MLVA/

http://www.efsa.europa.eu/en/efsajournal/pub/3556.htm

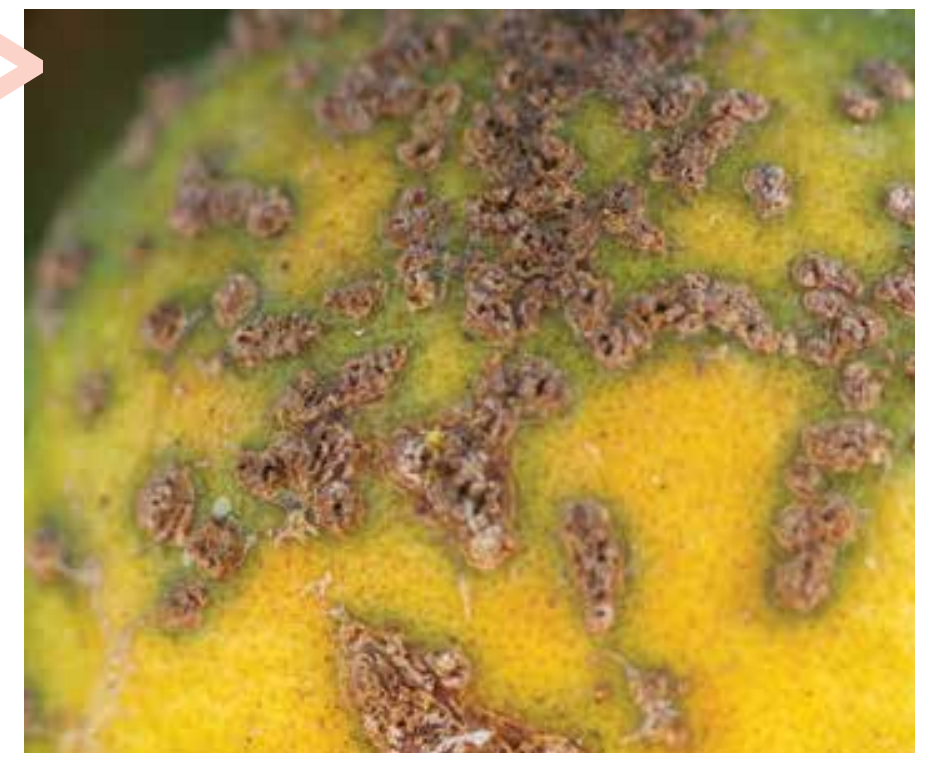

Chancre asiatique sur fruit. (c) 0. Pruvost/Cirada

\section{PARTENAIRES}

France > Agence nationale de la recherche $[A N R]$; Centre national de la recherche scientifique (CNRS) ; Conseil régional de la Réunion ; Fonds européen de développement économique et régional (Feder) ; Institut de recherche pour le développement (IRD) ; Université Paris-Sud.

\section{Maladies animales et zoonotiques}

\section{L'Europe et la Chine lancent LinkTADs}

En Chine, la production de produits animaux a quadruplé en 20 ans et les élevages se sont fortement intensifiés. Une situation qui interpelle sur la surveillance et le contrôle de maladies animales et zoonotiques comme les grippes aviaires ou la peste porcine africaine (PPA). L'émergence récente de la PPA en Russie fait craindre une arrivée prochaine en Europe et à plus long terme en Chine. La lutte contre la PPA illustre bien le besoin de collaboration et de coordination entre l'Europe et la Chine. C'est dans ce contexte que vient d'être lancé conjointement LinkTADs, un consortium de recherche, financé par l'Union Européenne et porté par la FAO, dont le volet « épidémiologie » est piloté par le Cirad.

\section{julien.cappelle@cirad.fr}

[Animal et Gestion Intégrée des Risques - Upr Agirs]

PARTENAIRES. Commission européenne, FAO. Chine > Beijing Chinese Center for Disease Control and Prevention (CADC) ; China Animal Health and Epidemiology Center [CAHEC) ; Harbin Veterinary Research Institute (HVRI) ; Huazhong Agricultural University (HZAU) ; Shanghai Veterinary Research Institute [SHVRI). Hongrie > Europa Media Non-profit Ltd. [EM]. Portugal > Sociedade Portuguesa de Inovação [SPI]. Royaume-Uni $>$ Royal Veterinary College (RVC). Suède $>$ National Veterinary Institute [SVA].

\section{http://ur-agirs.cirad.fr/projets/linktads}

\section{La Chine, numéro un de la production et de la consommation de produits animaux dans le monde.}

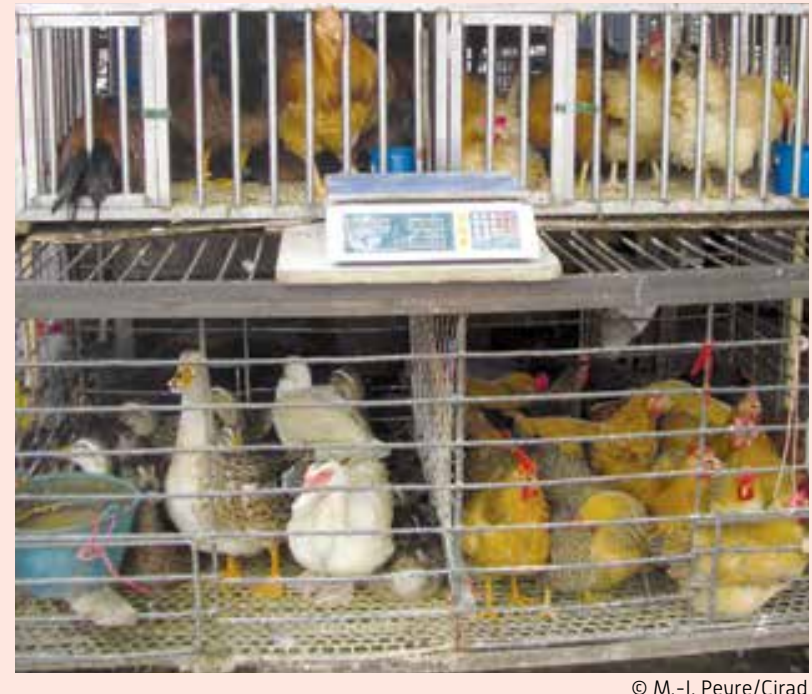




\title{
Maladies infectieuses
}

\section{"LA RÉPARTITION DES CHAUVES-SOURIS AFRICAINES INFLUE SUR LEUR DIVERSITÉ VIRALE »}

\author{
Une récente étude publiée dans PLoS One montre que la fragmentation de l'aire de répartition \\ des chauve-souris africaines est un facteur de leur richesse virale. Ces résultats vont aider à mieux \\ comprendre le rôle des chiroptères dans la circulation des maladies virales comme Ebola, Marburg ou la rage.
}

Entretien avec Mathieu Bourgarel, écologue au Cirad.

Pour mieux comprendre le rôle des chauves-souris dans la circulation de virus en Afrique, vous avez cherché les déterminants de la diversité virale chez ces animaux. Quels résultats avez-vous obtenus ?

Mathieu Bourgarel. La taille et le poids des animaux sont, sans surprise, deux facteurs significatifs. En revanche, plus étonnant, un indice élevé de la fragmentation de l'aire de distribution favorise la diversité de virus présente au sein d'une population de chauve-souris. Je me souviens que l'idée de tester ce critère est venue au cours d'une discussion avec deux de mes collègues, mais on ne s'attendait vraiment pas à ce qu'il ait une telle importance! Ce résultat devrait créer un précédent. Désormais, les scientifiques qui travaillent sur la richesse virale des animaux sauvages vont sans doute prendre en compte l'aire de distribution. Avec les rongeurs, les chiroptères représentent la majorité des mammifères sur terre. Les deux groupes sont largement impliqués dans la circulation des maladies infectieuses, sauf qu'il y a dix fois plus d'études menées sur les rongeurs que sur les chauves-souris.

\section{Des données ont été récoltées sur 15 espèces}

[8 Pteropodidae ou roussettes et 7 microchiroptères] en Afrique centrale et de l'Ouest. Comment avez-vous procédé ?

M. B. Des données de terrain ont été complétées par un vaste travail de récolte d'informations bibliographiques. Nous avons compilé des études issues de la littérature scientifique mais aussi des bases de données de I'Union internationale pour la conservation de la nature sur la distribution des espèces animales. A partir de cette synthèse, une base de donnée a été constituée avec, pour chaque population, des informations sur les traits morphologiques, écologiques et comportementaux. Ce travail collectif a été possible grâce à des nombreux partenariats avec notamment l'IRD, le Centre international de recherche médicale de Franceville (Gabon) ou I'Institut de virologie de Bonn (Allemagne)

Maganga G. et al. 2014. Bat distribution size or shape as determinant of viral richness in African bats. PLoS One, 9 : e100172. Doi : 10.1371/journal.pone.0100172

Gay N. et al., 2014. Parasite and Viral Species Richness of Southeast Asian Bats: Fragmentation of Area Distribution Matters. International Journal for Parasitology. Parasites and Wildlife 3 (2). Australian Society for Parasitology: 161-70. doi:10.1016/j. ijppaw.2014.06.003.

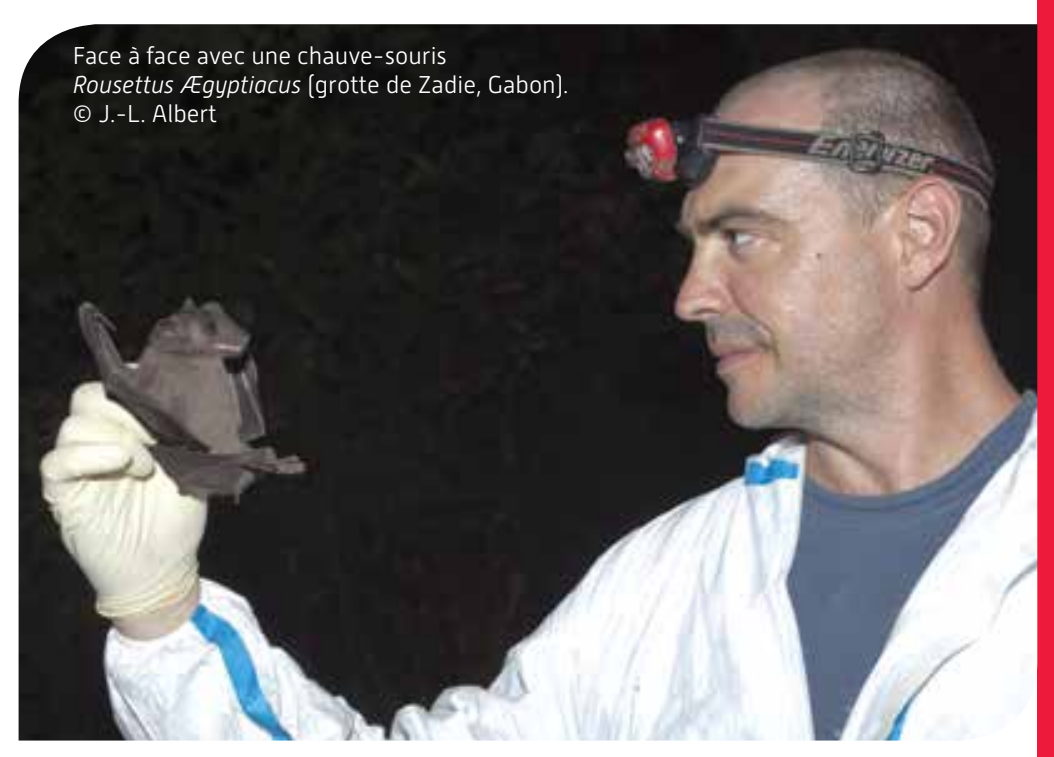

mathieu.bourgarel@cirad.fr

[Animal et Gestion Intégrée des Risques - UPR Agirs]

\section{Comment expliquez-vous vos résultats ?}

M. B. Les explications possibles ne sont encore que des hypothèses, il faudra d'autres études pour les vérifier ou les invalider. Le lien entre la fragmentation de l'aire de distribution et la richesse virale d'une population est la résultante de nombreux facteurs, dont des phénomènes de coévolution entre les virus et les chauves-souris. En outre, une aire de distribution morcelée peut signifier des habitats variés, cela pourrait expliquer que les chiroptères soient en contact avec une plus grande diversité de virus. Pour tester ces hypothèses, il nous faudra au préalable comprendre les « moteurs » sous-jacents de la fragmentation de l'aire de distribution d'une population animale, d'autant que cette corrélation n'est pas systématique. En effet, en Asie du Sud-Est, une étude comparable sur les chauves-souris a montré une tendance opposée. Cette différence pourrait s'expliquer par le fait qu'il reste moins de milieux naturels sur ce continent qu'en Afrique.

« La corrélation entre la fragmentation de la répartition géographique des chauves-souris et leur richesse viral a été une belle surprise ! " 


\title{
ACTION PUBLIQUE POUR LE DÉVELOPPEMENT
}

\section{Paiements pour services environnementaux [PSE] RESTAURER LA FORÊT EN RÉDUISANT LES INÉGALITÉS}

\author{
Une étude a comparé deux types de paiements pour services environnementaux [PSE] en termes d'efficacité, \\ d'efficience économique et d'équité. Les résultats suggèrent que si les PSE sont adaptés aux différentes \\ réalités des exploitations agricoles, ils peuvent non seulement restaurer les services écosystémiques, \\ mais aussi réduire la pauvreté.
}

Entretien avec Damien Jourdain, économiste au Cirad.

Vous avez comparé deux types de paiements pour services environnementaux [PSE]. Expliquez-nous...

Damien Jourdain. L'idée initiale des PSE est d'organiser un paiement par unité de terre forestière supposé correspondre aux services écologique rendus. En pratique, les paiements sont décidés sur la base d'un cout moyen d'opportunité des terres dans les zones de pentes et des capacités budgétaires des programmes. Dans les zones de montagne d'Asie du SudEst, les agriculteurs contrôlant le plus de terre, notamment irriguées, ont plus de facilité à y participer. Nous voulions trouver un moyen de lever le verrou d'accès pour les petits agriculteurs. Petites surfaces et accès restreint à l'eau forcent ces paysans à utiliser des modes de cultures qui favorisent l'érosion et incitent à défricher plus de terre à cause des faibles rendements. Sur la base de ces constats, nous avons comparé un PSE classique (PFF pour Payments for forests] avec un PSE qui inclue une aide à la construction de terrasses et à la mise en irrigation des parcelles (TFF pour Terraces for forests). Les résultats de nos simulations ont montré que ces programmes TFF augmentent la participation des fermiers les plus pauvres et produisent plus de zones reforestées par euro investi que des PSE de type PFF.

Vous avez utilisé la modélisation agricole pour analyser les programmes de paiements pour services environnementaux. En quoi cela consiste-il ?

D. J. Au préalable, nous avons réalisé des enquêtes et diagnostics de terrain sur les exploitations agricoles d'une région montagneuse au nord du Vietnam. Nous nous sommes ainsi constitués une base de données assez fine avec des renseignements comme le nombre d'hectares, les principales contraintes, les techniques de cultures, les intrants utilisés, les rendements, l'accès au crédit, etc. Rassemblées dans un modèle, ces informations nous ont permis de simuler les choix s'offrant à un agriculteur qui veut optimiser son activité. Ce modèle a été calibré pour ressembler à la réalité de terrain. Nous avons alors pu simuler l'impact des deux types de PSE, et les comparer suivant deux critères : l'équité et le rapport coût/ efficacité. Les résultats sont sans équivoque : le programme de type TFF est doublement plus performant. En maintenant les deux types de PSE, on pourrait concevoir un programme gagnant-gagnant qui permettrait à la fois d'augmenter les zones boisées et de réduire les inégalités entre les familles agricoles.

Est-ce novateur de prendre en compte l'équité dans les études concernant les PSE ?

D. J. Pas vraiment. Cela dit, s'il y a beaucoup d'études critiques, très peu proposent des alternatives plus équitables. Mais dans notre travail, l'idée innovante est plutôt celle de mettre à disposition plusieurs types de PSE qui seraient adaptés à la multiplicité des exploitations agricoles des zones de montagne d'Asie du Sud-Est. Dans notre cas, les programmes PFF sont destinés aux grandes surfaces cultivées et les TFF aux petits agriculteurs. II me semble important de maintenir ces deux types de PSE pour que le plus grand nombre puisse y avoir accès. Notre article montre que différents programmes de PSE adaptés et ajustés au contexte agricole, pourrait non seulement être utilisé pour restaurer des services écosystémiques, mais aussi réduire la pauvreté.

exploitations agricoles.

damien.jourdain@cirad.fr (Gestion de l'eau, acteurs et usages - UMR G-Eau)

Jourdain D. et al., 2014. Water for forests to restore environmental services and alleviate poverty in Vietnam: A farm modeling approach to analyze alternative PES programs. Land Use Policy, 41 : 423-437. Doi : 10.1016/j.landusepol.2014.06.024

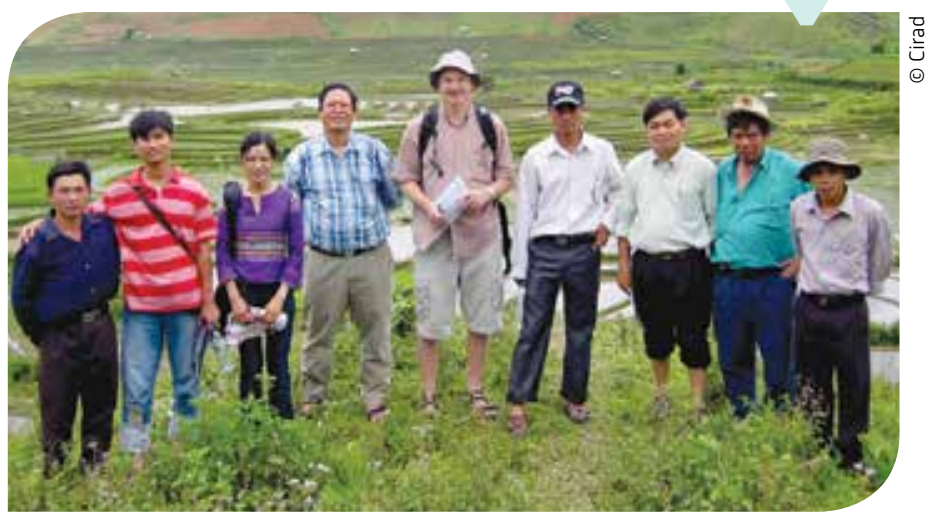




\section{DES INSTRUMENTS DE MARCHANDISATION} DE LA NATURE ?

\section{alain.karsenty@cirad.fr}

[Biens et services des écosystèmes forestiers tropicaux - UPR BSEF]

Les paiements pour services environnementaux sont fréquemment présentés comme des instruments "basés sur le marché » au motif qu'ils modifient les incitations, même si le recours à de véritables marchés est exceptionnel. Si sur un marché s'échangent avant tout des droits de propriété, ce n'est pas le cas avec la plupart des paiements pour services environnementaux, dans lesquels des droits d'usage sont suspendus contractuellement. C'est la thèse que défendent deux chercheurs du Cirad dans un ouvrage paru récemment. Selon eux, l'usage métaphorique du concept de marché est autant le fait des partisans du « tout-marché », pour disqualifier la réglementation, que de leurs adversaires, lesquels assimilent les incitations financières aux " marchés », sans que les uns et les autres n'explicitent jamais le concept qu'ils utilisent. Si les auteurs écartent l'hypothèse de marchandisation de la nature, ils soulignent néanmoins les risques liés à l'importance que pourraient prendre les motivations intéressées de conservation de la nature avec une généralisation de ces paiements.

\section{PARTENAIRE. France > Gret (France)}

L'instrumentation de l'action publique. Paris : Presses de Sciences Po, p. 161-189.

\section{Projet PESMIX \\ Atelier international à Montpellier}

Dans le cadre du projet PESMIX*, le Cirad, la mission Économie de la biodiversité de la CDC ainsi que leurs partenaires scientifiques ont organisé, avec le soutien de la région Languedoc-Roussillon, du 11 au 13 juin 2014, un atelier international intitulé : « Comment articuler les paiements pour services environnementaux aux autres instruments politiques et économiques, dans les pays du Sud et du Nord? ». Plus de 100 participants de tous horizons ont dressé un état des lieux des réflexions conceptuelles sur les PSE, des principaux retours d'expériences de mise en œuvre de l'instrument et des perspectives de développement.

alain.karsenty@cirad.fr

[Biens et services des écosystèmes forestiers tropicaux - UPR BSEF]

\section{http://pesmix.cirad.fr/atelier-2014}

* Le projet PESMIX est un projet de recherche financé par I'ANR et I'AIRD. Il est axé sur l'intégration de mécanismes de PSE au sein des instruments de politique environnementale au Mexique et à Madagascar.
Paysage agricole, Sulawesi, Indonésie. (c) B. Locatelli/Cirad

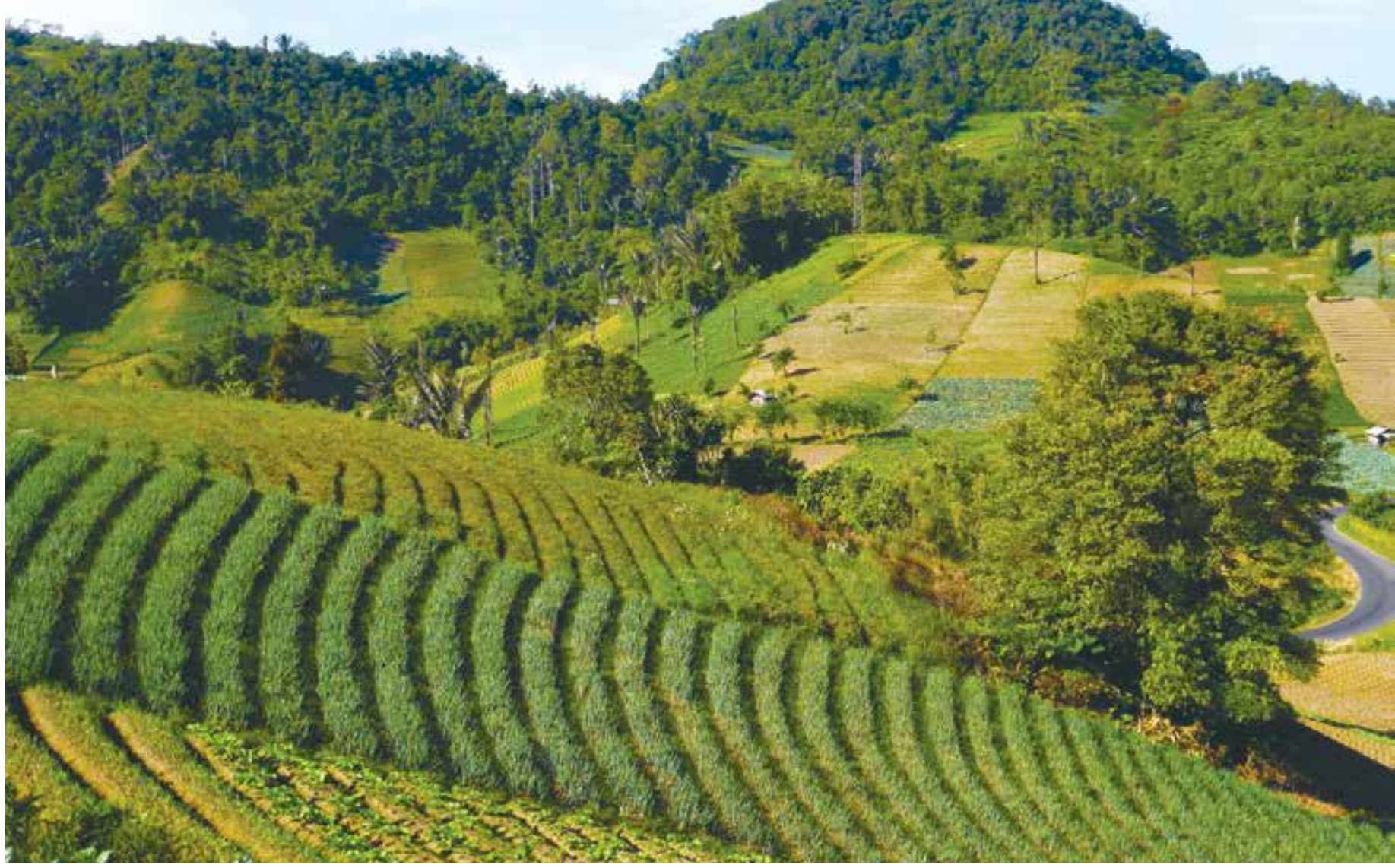




\section{PROMOUVOIR DES FORMES DURABLES DE DÉVELOPPEMENT EN AMAZONIE}

\author{
Le dispositif « Amazonie » fédère les activités \\ de recherche autour d'un objectif : concilier \\ préservation de l'environnement et appui
} aux populations rurales. Il vise à fournir l'expertise scientifique indispensable à la mise en place d'un développement territorial durable en favorisant l'augmentation de la productivité des systèmes de production familiaux.

PARTENAIRES. Brēsil > Embrapa Amazônia Oriental : réseau de stations de recherche et de techniciens sur les fronts pionniers; centre de Roraima ; Universidade Federaldo Para (UFPa] : Núcleo de Ciências Agrárias e Desenvolvimento Rural (NCADR) - laboratoire de recherche et d'enseignement sur l'agriculture familiale amazonienne ; Museo Paraense Emílio Goeldi : modélisation environnementale ; Universidade do Brasilia : Centre du développement durable(CDS). France > Cirad : UMR Art-Dev, Selmet, Innovation, Tetis ; UPR Aïda, BioWooEB, BSEF, Green.

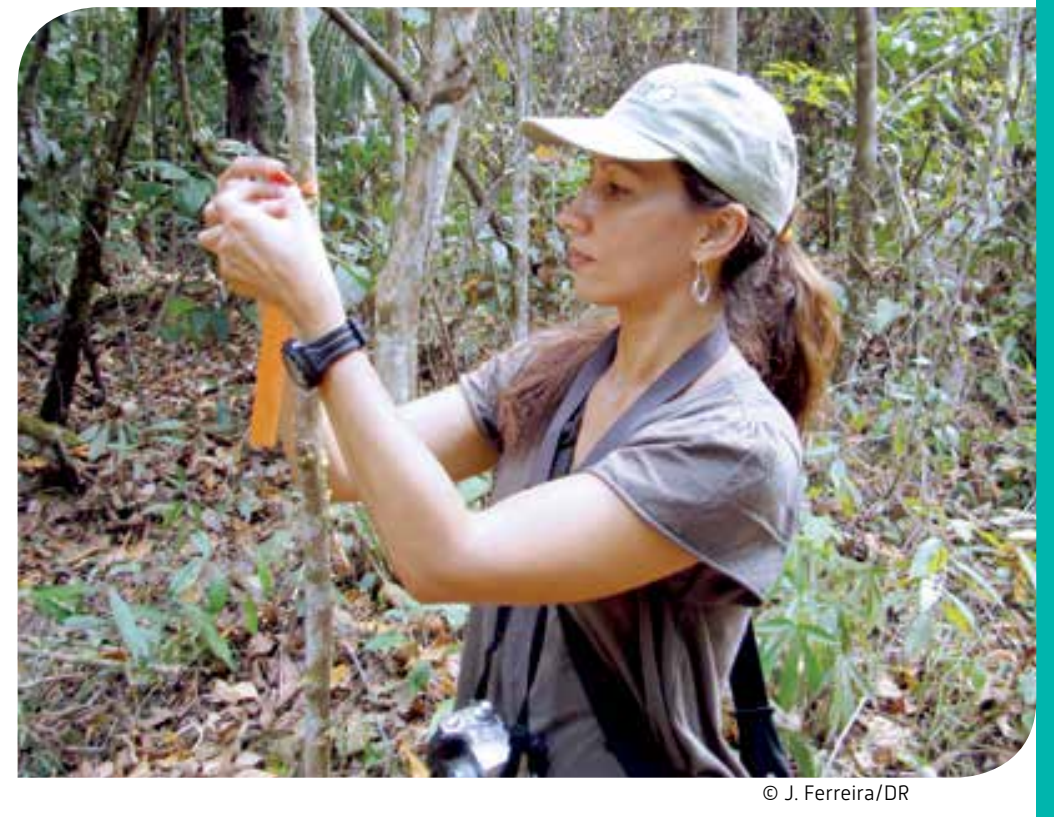

Joice Ferreira travaille à Belém au centre d'Amazonie orientale de l'Embrapa. Chercheur en écologie, elle est membre des Comités de pilotage du DP Amazonie et du projet Ecotera coordonnés par le Cirad.

joice.ferreira@embrapa.br

www.accord-biomeamazonien.org

\section{POUVEZ-VOUS PRÉSENTER}

\section{VOTRE PARTENARIAT}

\section{AVEC LE CIRAD ?}

Joice Ferreira. Je suis arrivée en 2006 alors que ce partenariat était déjà en place depuis longtemps, nos recherches étant parfaitement compatibles. L'Embrapa est une institution importante qui compte plus de 500 employés dans notre centre de Belém et nos challenges sont immenses. Avec le Cirad, nous partageons un objectif important qui est de contribuer au développement rural durable en Amazonie en préservant l'équité sociale, la conservation des forêts et les débouchés pour les populations locales. Nous collaborons sur plusieurs fronts mais je pourrais citer trois thématiques de recherche pour lesquelles nous travaillons étroitement ensemble : les services environnementaux, la gestion forestière et le développement territorial.

\section{QUELS SONT VOS PROJETS \\ EN COURS?}

J. F. Deux projets principaux sont en cours. Ecotera* Eco-efficiences et développement territorial en Amazonie Brésilienne] est un projet de recherche-développement à Paragominas, premier Municipe Vert d'Amazonie. TmFO** (Observatoire des forêts tropicales aménagées], un réseau pan-tropical incluant I'Amazonie [Brésil, Guyane française, Guyana, Surinam, Bolivie et Pérou) et qui s'intéresse à la résilience des forêts tropicales exploitées. Dans le cadre de RAS "Sustainable Amazon Network» que je coordonne, nous étudions les impacts écologiques et sociaux de la déforestation et de la dégradation forestière. Nous avons aussi co-organisé plusieurs évènements scientifiques et des formations. Actuellement, nous co-organisons un séminaire sur la dégradation des forêts, qui aura lieu à Belém en avril 2015. Chercheurs et décideurs se retrouveront autour d'une table pour discuter des menaces importantes pesant sur les forêts amazoniennes et que les politiques actuelles ne prennent toujours pas en considération.

\section{QU'APPORTE EN PLUS LE CIRAD COMME PARTENAIRE?}

J. F. Entre les deux institutions, il existe une grande complémentarité des compétences, ce qui nous aide à renforcer nos équipes. Nous bénéficions grandement de ce partage d'expériences. Les deux institutions, qui s'impliquent fortement pour augmenter l'impact de leurs recherches à travers un réseau de scientifiques, en particulier avec I'Université fédérale du Para, travaillent en synergie. Notre partenariat est une réussite. A court et long teme, il est prometteur pour les deux parties.

* Coordonné par Marie-Gabrielle Piketty, UPR Green, Gestion des ressources renouvelables et environnement.

** Coordonné par Plinio Sist, UPR BSEF, Biens et services des écosystèmes forestiers tropicaux. 


\section{SOCIÉTÉS, NATURE ET TERRITOIRES}

\section{Au-delà de la vulnérabilité LA RÉSILIENCE DES SOCIO-ÉCOSYSTĖMES}

\section{Comment, dans son environnement, une société appréhende des perturbations et y réagit ? Pour répondre à cette question préoccupante, dans un contexte de changements globaux, une communauté scientifique interdisciplinaire dont le Cirad fait partie s'est constituée depuis une dizaine d'années autour de la "résilience des socio-écosystèmes" (Resilience Alliance). Plus récemment, ce concept a été largement adopté pour repenser la réponse à apporter aux crises récurrentes et diverses qui touchent les pays les plus pauvres.}

Entretien avec Aurélie Botta, écologue au Cirad

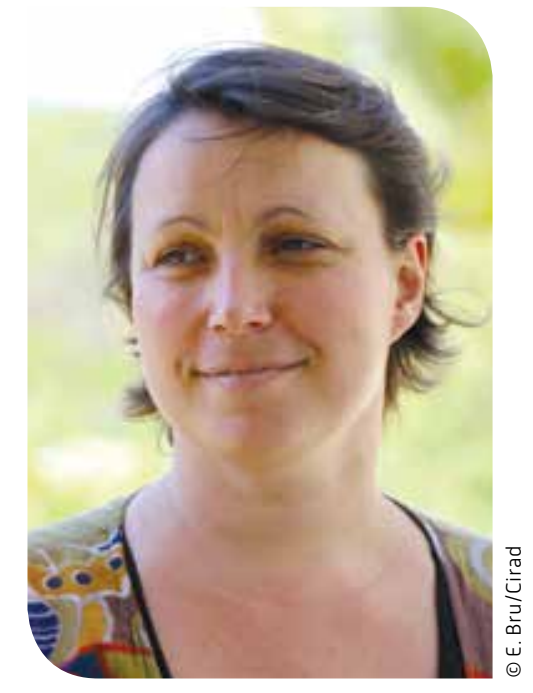

Qu'est-ce que la résilience des sociétés dans leur environnement ?

Aurélie Botta. Il s'agit de savoir comment une société dans son environnement - un socio-écosystème - appréhende des perturbations, s'y prépare et y réagit à court, moyen et long termes. Initialement, le concept a été développé en sciences physiques pour qualifier la capacité d'un matériau à retrouver ses propriétés après un choc (chimique, mécanique ou autre). Dans les années 1960, des psychiatres et des psychanalystes, comme Boris Cyrulnik en France, ont appliqué ce concept aux individus ayant subi un traumatisme pour évaluer et développer leurs capacités à se reconstruire et vivre avec cette histoire. Parallèlement, des écologues et des mathématiciens s'en sont emparé pour analyser l'évolution d'écosystème soumis à des perturbations : des forêts à la suite d'un incendie, une mangrove après un ouragan. La résilience des socio-écosystèmes réintroduit l'humain dans l'analyse, les perturbations comme les mécanismes de réponse impliquant de fait la société et environnement (e.g. une crise alimentaire créée par des échanges internationaux inadaptés, une adaptation via l'institution de nouvelles règles de partage des ressources).

Que sait-on aujourd'hui de la résilience des socio-écosystèmes ? A. B. La résilience n'est pas systématiquement «bonne». Questionner la résilience d'un socio-écosystème c'est chercher à distinguer différentes options qui s'offrent à une société et son environnement face aux perturbations. On peut chercher à préserver le fonctionnement de ce socio-écosystème même si certains aspects de l'environnement ou de la société changent, on parle alors d'adaptation. On construit une digue pour se prévenir des crues par exemple suite aux variations climatiques. Mais on peut également avoir intérêt à profiter de la perturbation pour transformer les interactions société-environnement non souhaitables. La perturbation est alors une opportunité de changement, comme c'est le cas avec certains excès de la révolution verte qui conduisent aujourd'hui à une transformation profonde des pratiques et des institutions d'où émerge l'agriculture écologiquement intensive ou agroécologie.

La capacité d'un socio-écosystème à s'adapter ou à se transformer dépend de sa capacité à s'auto-organiser et à apprendre, que la perturbation soit connue ou nouvelle, abrupte ou progressive, exogène ou endogène. Enfin, dans un monde toujours plus connecté, les perturbations comme la réponse que leurs apportent les socio-écosystèmes sont également interdépendantes dans l'espace et le temps.

\section{La résilience et le développement, est-ce un sujet fédérateur aujourd'hui ?}

A. B. : Le concept de résilience considère que le changement n'est pas exceptionnel mais intrinsèque, et qu'il ne s'agit plus de réfléchir en terme de stabilité et d'équilibre mais en terme d'orientation de trajectoire. C'est justement pour réfléchir l'articulation entre gestion de crise à court terme et trajectoire de développement à long terme que de nombreux acteurs issus du monde du développement (Banque mondiale, FAO, CGIAR, AFD) se sont saisis du concept de résilience. Toutefois la résilience des uns n'est pas celle des autres. Doit-on cibler les plus pauvres ou considérer tout le système du fait de potentiels transferts de vulnérabilité ? Se focalise-t-on sur les stratégies d'adaptation ou envisage-t-on des transformations drastiques ? Prend t-on en compte la complexité des feedbacks et seuils écologiques? C'est à travers le prisme du dialogue entre les différentes communautés et écoles que François Bousquet et moi-même avons coordonné l'organisation du colloque Resilience 2014 afin de mettre en valeur les apports réciproques de ces différentes approches.

\section{Resilience2014 www.resilience2014.org}




\section{Forêts aménagées}

Un réseau mondial pour mesurer leur résilience

La forêt tropicale primaire, vierge de toute perturbation humaine, captive l'imaginaire des gens tout comme celui des chercheurs. Son exploitation, souvent pratiquée de façon prédatrice sans le souci de maintenir sur le long terme ses ressources et ses services (biodiversité, stockage de carbone) a plutôt mauvaise presse. Pourtant, le concept d'exploitation raisonnée fait son chemin parmi les forestiers et constitue un défı majeur dans la préservation des forêts tropicales de demain. Un réseau mondial d'institutions, l'Observatoire des forêts tropicales aménagées, étudie pour la première fois, à l'échelle régionale et pantropicale, la résilience de ces forêts après abattage sélectif. Trois grandes régions forestières sont concernées : Bassin amazonien, Bassins du Congo et du Sud-Est asiatique. Le projet, coordonné par le Cirad et financé par le programme Forest Tree and Agroforestry du CGIAR, implique vingt-quatre institutions de recherche provenant de seize pays différents.

\section{http://tmfo.org/}

plinio.sist@cirad.fr

(Biens et services des écosystèmes forestiers tropicaux - UPR BSEF]

\section{0 millions d'ha}

des forêts tropicales
destinés à la
production de bois,
soit $10 \%$ des
forêts mondiales.

\section{COSTA RICA BIODIVERSITÉ ET SERVICES ÉCOSYSTÉMIQUES FONT BON MÉNAGE}

bruno.locatelli@cirad.fr [Biens et services des écosystèmes forestiers tropicaux - UPR BSEF]

Les zones identifiées comme importantes pour la biodiversité peuvent aussi l'être pour d'autres services écosystémiques comme le stockage du carbone, la conservation de l'eau et la qualité paysagère. Ce n'est pas forcément le cas des zones qui renferment de grandes quantités de carbone, qui, elles, fournissent des avantages moindres pour les autres services écosystémiques. Ce sont les conclusions d'une étude menée au Costa Rica par une équipe du Cirad, du Cifor et du Catie. Des résultats qui pourraient avoir des répercussions sur le choix des zones à protéger dans le cadre des initiatives visant à atténuer les émissions dues à la déforestation comme la REDD. Privilégier les régions de forte biodiversité, tenir compte de la multi-

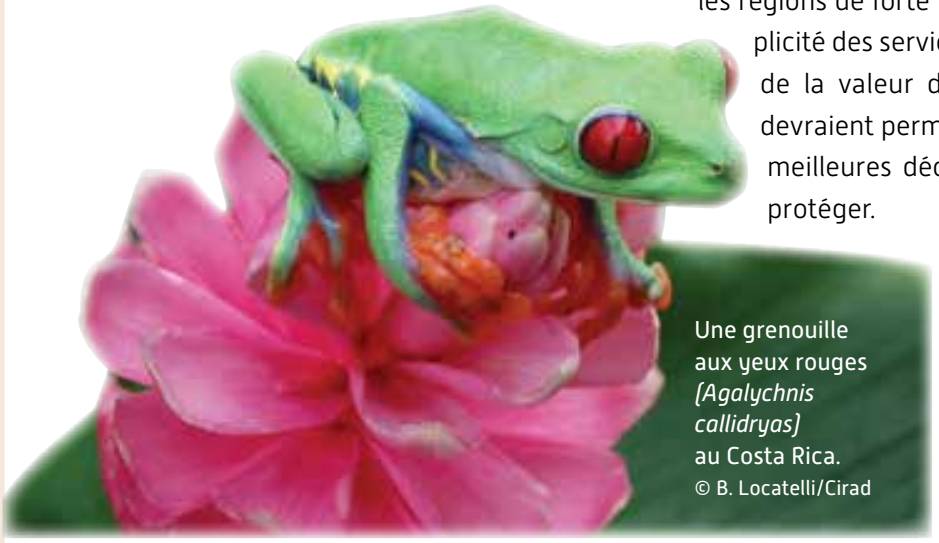

PARTENAIRES. Indonésie > Centre for International Forestry Research [Cifor]. Costa Rica > Centro Agronómico Tropical de Investigación y Enseñanza [Catie)

Environmental Conservation, $41: 27-36$ Doi : $10.1017 / 50376892913000234$

\section{FORÊTS TROPICALES HUMIDES \\ LE CHANGEMENT CLIMATIQUE POURRAIT ENTRAIINER UNE BAISSE DE LA PRODUCTIVITÉ}

bruno.herault@cirad.fr (Ecologie des forêts de Guyane - UMR Ecofog)

Les forêts tropicales humides sont menacées sur une échelle sans précédent par les changements globaux. Les températures augmentent et devraient continuer d'augmenter dans les prochaines décennies alors que les précipitations vont diminuer. Comment les arbres réagissent-ils à ces perturbations ? Quelles répercussions ont-elles sur leur croissance ? Et quels sont les facteurs climatiques auxquels cette croissance est le plus sensible ? De la réponse à ces questions dépend la productivité future des forêts tropicales humides. Une analyse des données recueillies sur toute la zone tropicale a permis à une équipe du Cirad de clarifier la situation. Elle montre, en effet, que la croissance des arbres présente une très forte saisonnalité et que sa variabilité s'explique principalement par un effet du site. L'analyse révèle aussi que ce sont les précipitations et le rayonnement solaire qui sont les déterminants majeurs de cette croissance. La productivité des forêts tropicales humides pourrait donc diminuer dans les années à venir si, comme les modèles climatiques le prévoient, les précipitations baissent et les sécheresses deviennent plus fréquentes.

PARTENAIRES. Allemagne $>$ Université de Hohenheim. Belgique $>\mathrm{KU}$ Leuven. Brēsil > Instituto Nacional de Pesquisas Espaciais (Inpel). Italie $>$ Euro-Mediterranean Centre for Climate Change. France > Institut national de la recherche agronomique (Inra) ; Université des Antilles et de la Guyane

PLoS One, 9 : e9233. Doi : 10.1371/journal.pone.0092337

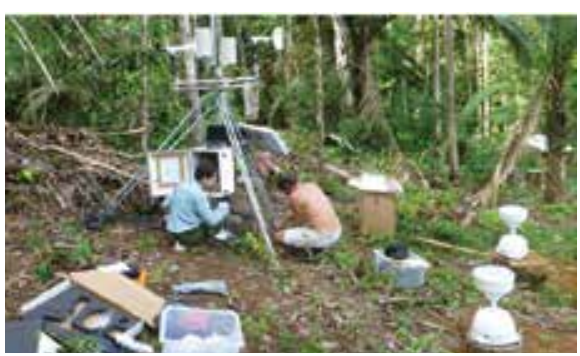

Installation d'une station micrométéorologique, à Réserve de la Trinité, en Guyane française. (c) B. Hérault/Cirad 


\section{PARTENARIAT SCIENTIFIQUE} MULTI-INSTITUTIONNEL ET AGRICULTURES IRRIGUÉES AU MAGHREB

Le réseau de compétence Sirma - Systèmes irrigués au Maghreb -, qui réunit une soixantaine de chercheurs, et d'enseignants-chercheurs, associe des institutions en Algérie, au Maroc, en Tunisie et en France autour d'un domaine « Les économies d'eau dans les systèmes irrigués au Maghreb ". L'objectif est triple : la recherche, l'enseignement supérieur et l'appui au développement.

POUVEZ-VOUS PRÉSENTER SIRMA EN QUELQUES MOTS ?

Ali Hammani. Sirma est né de la volonté de scientifiques maghrébins et français d'initier des échanges dans le cadre de la recherche agronomique, plus particulièrement sur la gestion de l'eau en agriculture et les systèmes d'irrigation. En 2004, ce dispositif développé par le Cirad et ses partenaires a reçu le feu vert du ministère français des Affaires étrangères avec un montant alloué de $2000 \mathrm{k€}$ pour une durée de cinq ans. Une équipe multidiciplinaire s'est alors constituée autour de la thématique de l'économie d'eau en systèmes irrigués au Maghreb. Depuis, Sirma est devenu une sorte de label sur lequel on capitalise. II a permis de fédérer des moyens et c'est une grande richesse en termes de production scientifique.

\section{QUELLE EST LA VALEUR A JOUTÉE DU CIRAD POUR LE DISPOSITIF ?}

A.H. Nous travaillons en symbiose. Le Cirad a une approche intéressante du Maghreb, sur les questions prioritaires. Et l'utilisation de l'eau en agriculture en fait partie. Nous pouvons ainsi croiser nos expériences. II remplit le rôle de coordinateur et nous profitons de son réseau. C'est aussi un appui important dans la formation. Vingt-deux thèses de doctorat ont été soutenues ou sont en cours, et plus de 60 étudiants ont fait un master.Ce partenariat nous apporte la reconnaissance nationale et internationale, et une visibilité nécessaire aux financements de projets.

\section{DES PROJETS EN PERSPECTIVE ?}

A.H. : Pour moi, c'est surtout continuer à avancer ensemble. Plus concrètement, nous avons des projets communs en recherche/développement et enseignement supérieur et nous sommes toujours à l'affût de nouveaux projets qui pourront pérenniser notre collaboration future. A terme, notre objectif est de constituer un pôle d'excellence dans la formation et la recherche sur les agricultures irriguées au Maghreb.

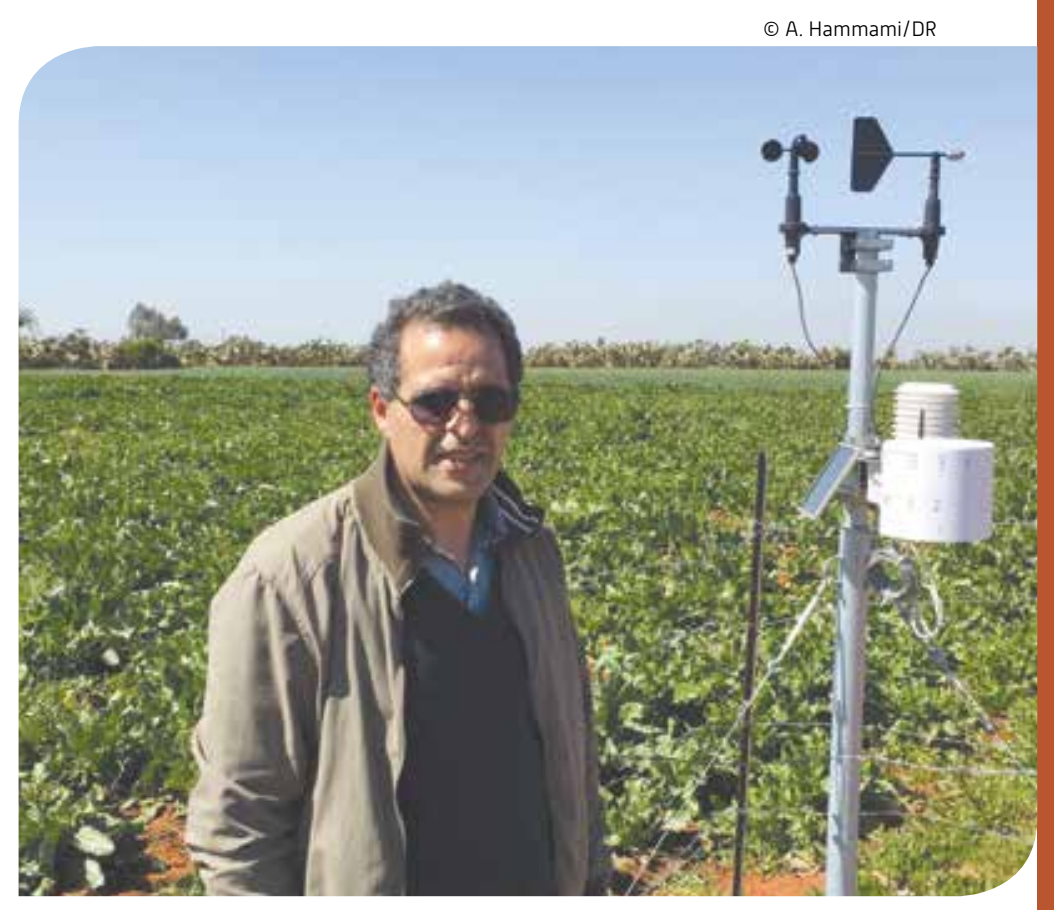

Ali Hammani est enseignant-chercheur, chef du département Eau, Environnement et Infrastructures à I'Institut agronomique et vétérinaire (IAV) Hassan II à Rabat. Il était membre du Comité de Programme du Département Environnements et Sociétés du Cirad entre 2011 et 2 à14. II participe au réseau Sirma en tant que coordinateur scientifique du projet à I'IAV.

ali.hammani@gmail.com

www.rcp.sirma.org

PARTENAIRES. Algérie > Centre de Recherche Scientifique et Technique sur les Régions Arides/Université Mohamed Khider, Biskra ; Ecole nationale supérieure agronomique d'Alger ; Université de Khémis Miliana. France > Cirad ; Centre International de Hautes Etudes Agronomiques Méditerranéennes - Institut agronomique méditerranéen de Montpellier (Ciheam - lamm) ; Centre international d'études supérieures en sciences agronomiques (Supagro Montpellier) ; Institut de recherche pour le développement (Ird) ; Institut des sciences et industries du vivant et de l'environnement (AgroParisTech) ; Institut national de recherche en sciences et technologies pour l'environnement et l'agriculture (Irstea). Maroc > Ecole Nationale d'Agriculture de Meknes (EnaMeknes) ; Institut Agronomique et Vétérinaire Hassanll de Rabat (IAV Hassan II). Tunisie > Institut national d'agronomie de Tunis (Inat) ; Institut National de Recherche en Génie Rural Eaux et Forêts (Inrgref), Tunis.

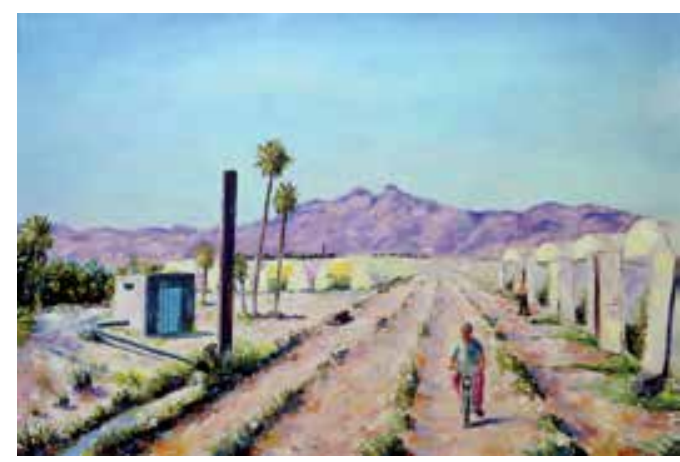

Tableau réalisé pour le DP Sirma (c) Faycel Berkat 


\section{INDICATEURS 2014}

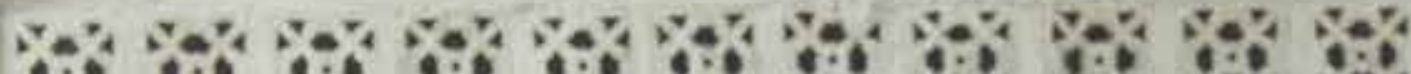

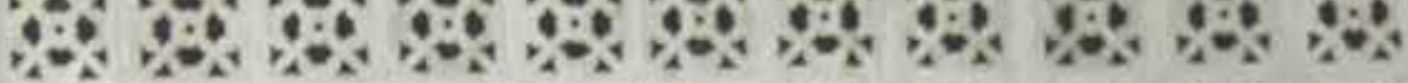

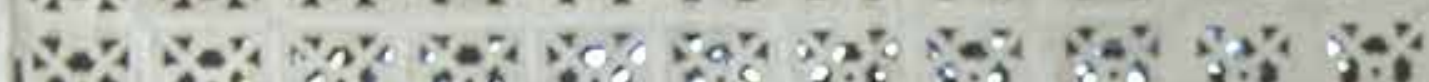

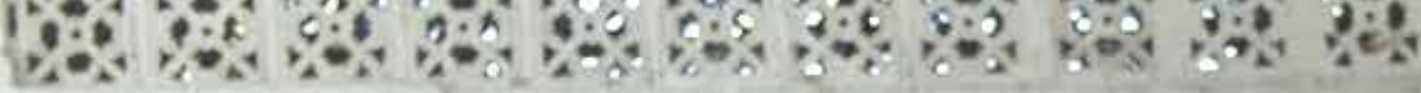

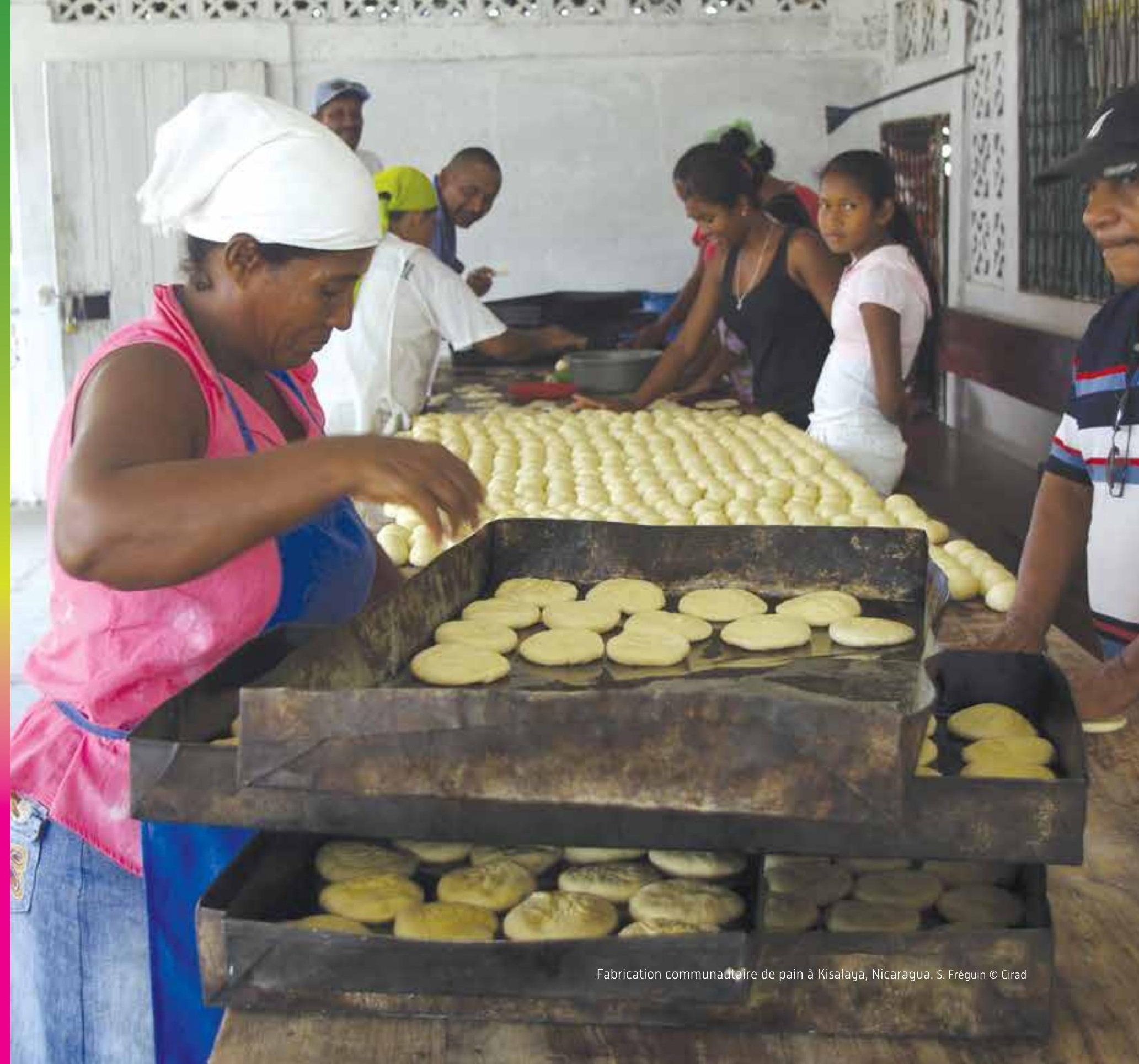




\section{> L'AMBITION D'UNE SCIENCE PARTAGÉE EN RÉPONSE AUX DÉFIS DU SUD}

Comme l'indique le document Objectifs de stratégie scientifique et partenariale (OSSP) 2014-20193, la mobilisation des équipes autour des six axes stratégiques, réactualisés, ainsi que les actions de renforcement des compétences au Sud sont au cœur des ambitions du Cirad.

En 2014, le Cirad a poursuivi ses efforts de production scientifique de qualité avec la publication d'articles dans des revues évaluées par les pairs sur des sujets s'inscrivant dans les priorités scientifiques de l'établissement et explorant les nouveaux fronts de recherche. L'essentiel des publications du Cirad sont référencées dans les axes comme en témoigne la figure ci-dessous. Les articles de revues à comité de lecture se répartissent principalement entre les axes Agriculture écologiquement intensive (36\%) et Santé des animaux et des plantes (25\%).

\section{Répartition, par axes stratégiques, des articles de revues à comité de lecture, avec ou sans facteur d'impact $^{3}$}

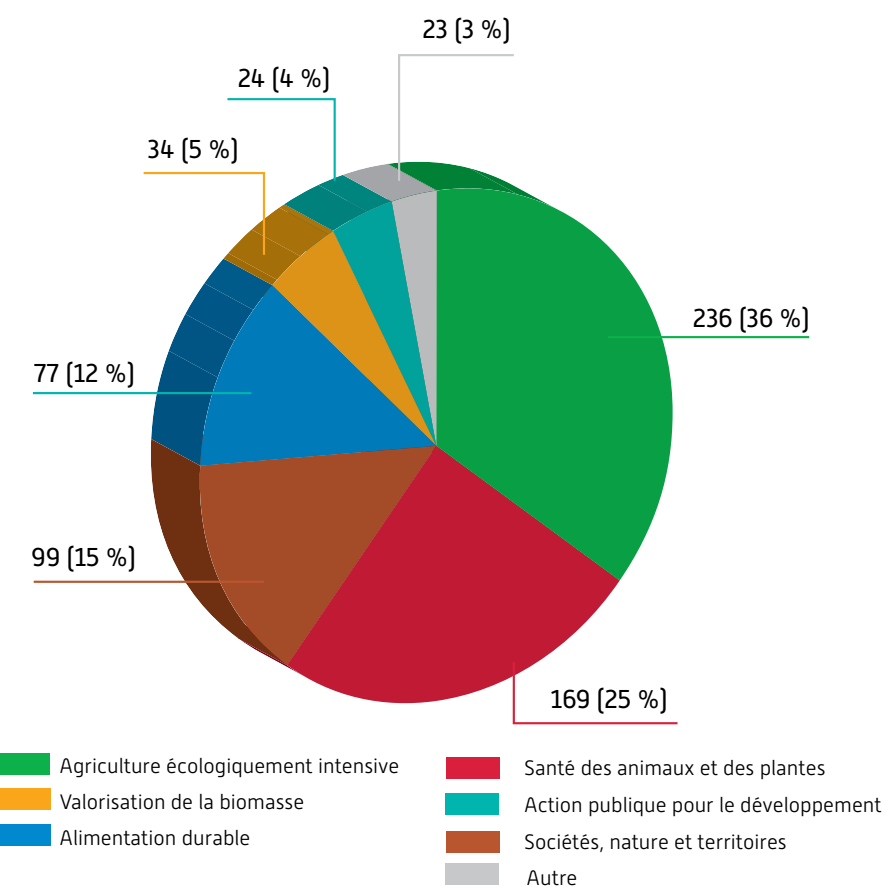

Source : Agritrop. Dist. DGD-RS. Les données 2014 sont partielles : elles reflètent l'état de la base Agritrop au 28 février 2015

1. Ambition 1. Faire référence au niveau mondial sur nos priorités scientifiques. Ambition 2 Co-construire des partenariats stratégiques de recherche agronomique pour le développement. Ambition 3. Développer les conditions d'une innovation efficace. Ambition 4. Evoluer pour assumer nos ambitions.

2. http://www.cirad.fr/qui-sommes-nous/notre-strategie
L'augmentation du nombre de copublications avec au moins un auteur du Sud entre 2010 et 2014 (de 328 à 391) reflète l'orientation du Cirad vers un partenariat équilibré et durable avec ses partenaires scientifiques du Sud. Ces copublications sont majoritaires [51\%] en 2014.

Après une année de baisse en 2013 (-12,8 \%), liée en partie à la mobilisation suscitée par la vague d'évaluation par l'Aeres de toutes les unités, le nombre de doctorants encadrés au Sud a connu un accroissement significatif $[+26 \%]^{4}$.

\section{Copublications avec des acteurs du Sud}

Un pays est qualifié de pays du Sud lorsqu'il figure sur la liste OCDE/CAD des pays bénéficiant de l'aide publique au développement.

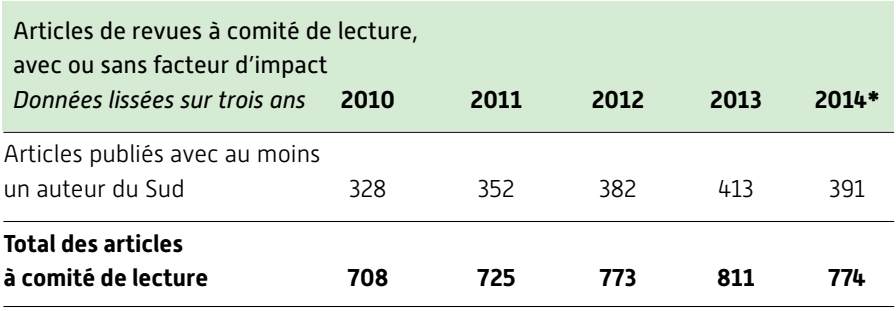

Source : Agritrop. Dist. DGD-RS. Les données 2014 sont partielles : elles reflètent l'état de la base Agritrop au 28 février 2015. * 2014 : données provisoires.

Encadrement des thésards par les chercheurs du Cirad (Source : DGD-RS)

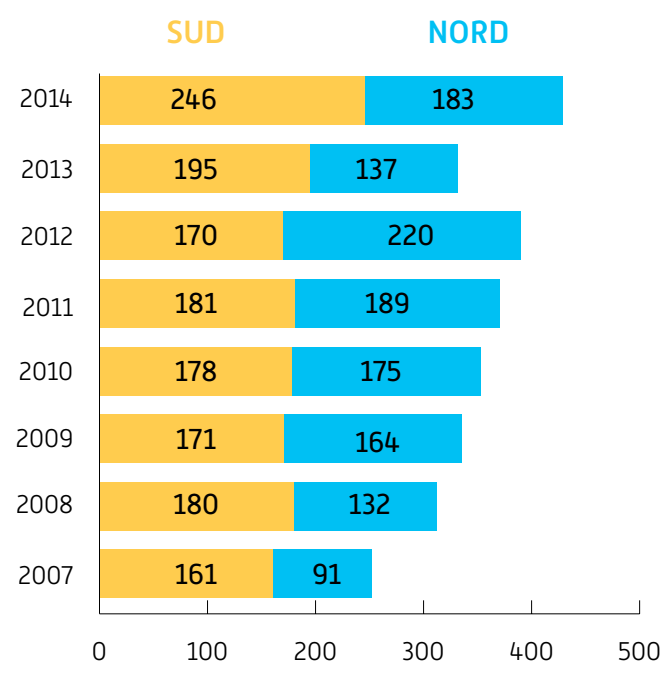

3. Suite à la redéfinition des axes stratégiques à partir de l'année 2014, seules les données de publications 2014 peuvent être restituées.

4. Par ailleurs, le système de suivi des doctorants a fait l'objet d'amélioration de traitement des informations issues de différentes sources. 


\section{> UNE PRODUCTION SCIENTIFIQUE DIVERSIFIÉE ET DE QUALITÉ}

Le Cirad entend porter la science pour le développement au meilleur niveau scientifique mondial en la gardant ancrée dans les questionnements, les terrains et ses partenariats au Sud. Aussi, a-t-il poursuivi son effort en matière de qualité de ses publications scientifiques, de compétitivité de ses équipes et de diversité de ses productions vers ses différents publics.

Pour la période 2013-2014, les articles publiés dans les revues à facteur d'impact (1 211 soit $33 \%$ ) et les communications de congrès (1 369 soit $37 \%$ du total des publications] sont les publications les plus nombreuses.

\section{Répartition, par type de document, des publications du Cirad pour la période 2013-2014}

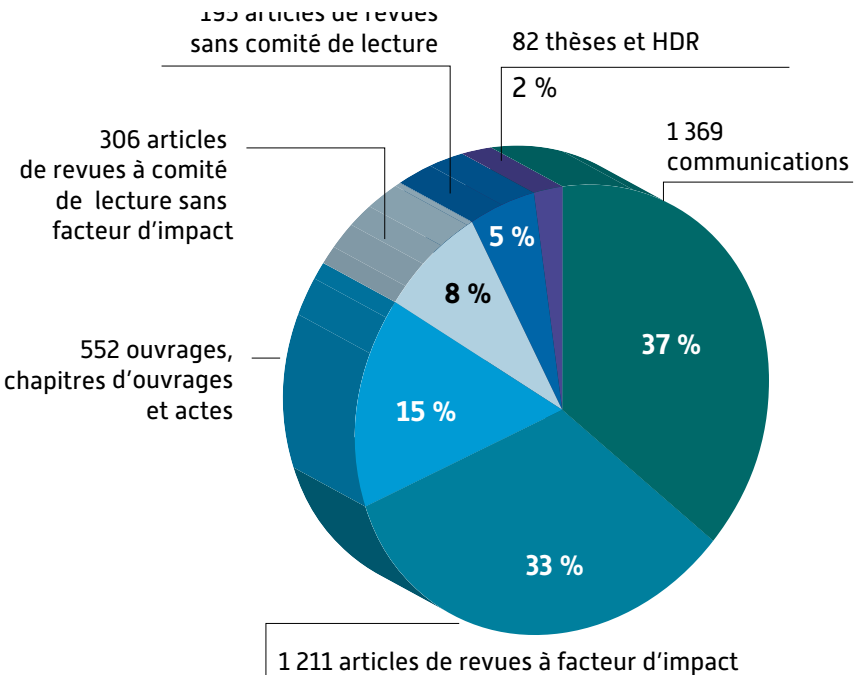

Source : Agritrop. Dist. DGD-RS. Les données 2014 sont partielles : elles reflètent l'état de la base Agritrop au 28 février 2015

\section{La formation à la recherche des cadres scientifiques du Cirad} Nombre de HDR au Cirad

\begin{tabular}{cccccc}
2009 & 2010 & 2011 & 2012 & 2013 & 2014 \\
\hline 107 & 129 & 136 & 143 & 166 & 162 \\
\hline
\end{tabular}

Source : SIRH, DGD-RD
Après plusieurs années de progression, le nombre des articles de revues à comité de lecture, avec ou sans facteur d'impact, est stable depuis deux ans. De même, le nombre de HDR après une période de forte augmentation est stable sur les deux dernières années. Les activités de valorisation sont, elles aussi, stables. L'évolution, ces dernières années, concerne l'orientation vers le co-dépôt avec les partenaires industriels ou publics qui contribuent au renforcement des réseaux de l'établissement et au partage des risques financiers liés à ces activités.

\section{Evolution des articles de revues entre 2010 et 2014}

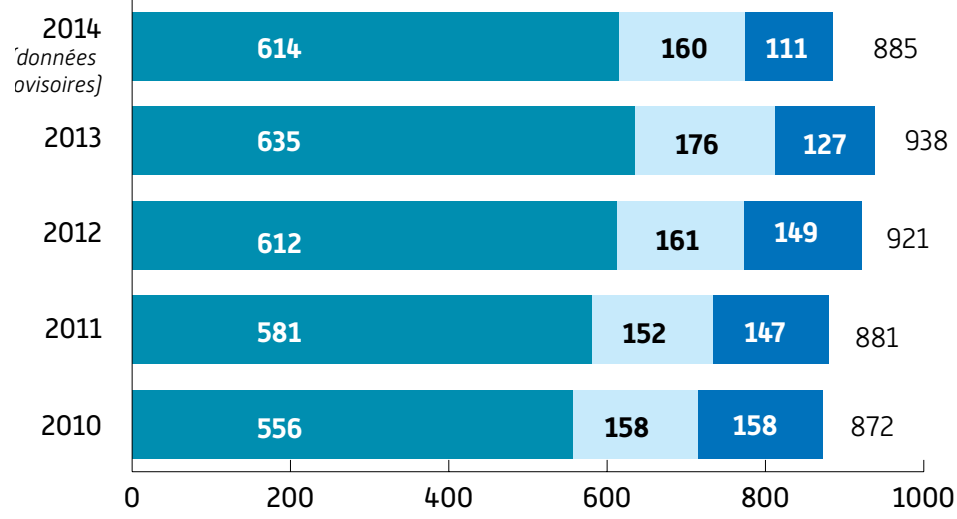

Articles de revues à facteur d'impact

Articles de revues à comité de lecture sans facteur d'impact

Articles de revues sans comité de lecture

\section{La valorisation : brevets, certificats d'obtention végétale et logiciels}

Nombre de brevets, certificats d'obtention végétale et logiciels déposés (dont nombre de brevets]

\begin{tabular}{lllllll}
2008 & 2009 & 2010 & 2011 & 2012 & 2013 & 2014 \\
\hline $13(0)$ & $26(4)$ & $10[5]$ & $8(2)$ & $8(3)$ & $9(0)$ & $8[3]$ \\
\hline
\end{tabular}

Source : DelValo, DCD-RS 


\section{> UNE RECHERCHE AGRONOMIQUE NATIONALE OUVERTE SUR L'EUROPE ET LE RESTE DU MONDE}

Le Cirad a poursuivi son action aux différents niveaux : au niveau régional français, notamment à travers la mise en place des politiques de site ; au niveau national, en particulier par son rapprochement avec l'Inra sur les plans scientifiques et de la coopération internationale ainsi qu'avec les autres organismes de recherche et d'enseignement français dans le domaine de l'environnement et de l'agriculture dans le cadre de l'alliance AllEnvi et au sein du consortium national Agreenium; au niveau européen avec des actions clés (IntensAfrica) de structuration des acteurs de la RAD; et au niveau international avec comme instrument privilégié, les 21 dispositifs en partenariat [DP] et par le renforcement des actions engagées avec le Groupe consultatif de la recherche agronomique internationale (GCRAI).

Le nombre des co-publications du Cirad avec d'autres institutions continue de progresser. Plus de la moitié (58\%) des articles de revue à comité de lecture, avec ou sans facteur d'impact, sont co-publiés avec une institution à l'international illustrant la priorité au Sud, à l'Europe et à l'international.
La mobilité géographique des Ciradiens est globalement stable sur 20132014 de même que le nombre de cadres scientifiques affectés dans les dispositifs en partenariat (DP) et dans les Dom, après une progression significative dans les DP entre 2012 et 2013. Parmi les 21 DP, 11 sont localisés en Afrique (incluant 1 DP basé dans les Dom), 5 en Amérique Latine, 4 en Asie, 1 en Méditerranée. Le nombre de missions baisse légèrement, la destination principale restant l'Afrique subsaharienne.

Dans le contexte particulier de première année de mise en œuvre du nouveau programme H2020, le Cirad a connu en 2014 un faible taux de succès aux appels d'offres du PCRDT. Cependant, le Cirad renforce par ailleurs sa mobilisation sur d'autres types de financements européens tels que les instruments de la DG Développement et coopération (DevCo)où la place de la recherche agronomique est de plus en plus reconnue.

\section{Evolution des co-publications du Cirad entre 2010 et 2014}

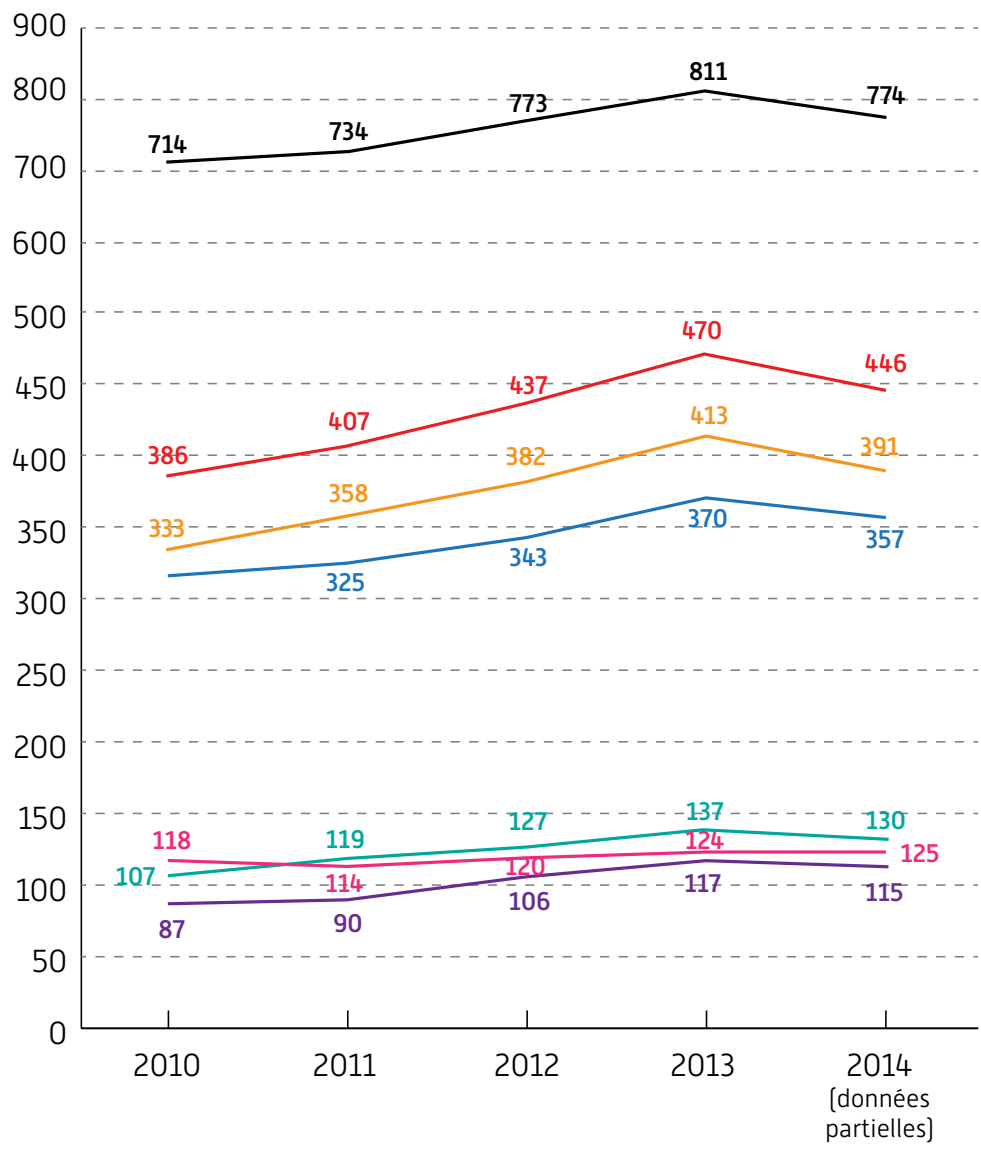

Articles publiés avec une institution internationale, hors Union européenne*

Articles publiés avec une institution d'un pays du sud

Articles publiés avec une institution en France

Articles publiés avec l'Inra

Articles publiés avec une institution de l'Union européenne, hors France

Articles publiés avec une institution d'un pays du Nord, hors Union européenne

Nombre total des articles de revues à comité de lecture, avec ou sans facteur d'impact**

[*] Une institution à l'international est une institution qui n'est pas localisée en France. Elle est localisée dans un pays du Sud ou dans un pays du Nord.

[**) Les co-publications sont calculées en compte de présence. Le compte de présence mesure la « participation » de l'institution à la production scientifique : l'institution est créditée d'une participation unitaire à une publication dès lors que sa présence dans la publication est attestée par son adresse. De ce fait, les valeurs obtenues pour les acteurs en France, de l'UE ou à l'international ne sont pas additives. 


\section{Répartition des affectations hors métropole, selon les destinations [en équivalent temps plein]}

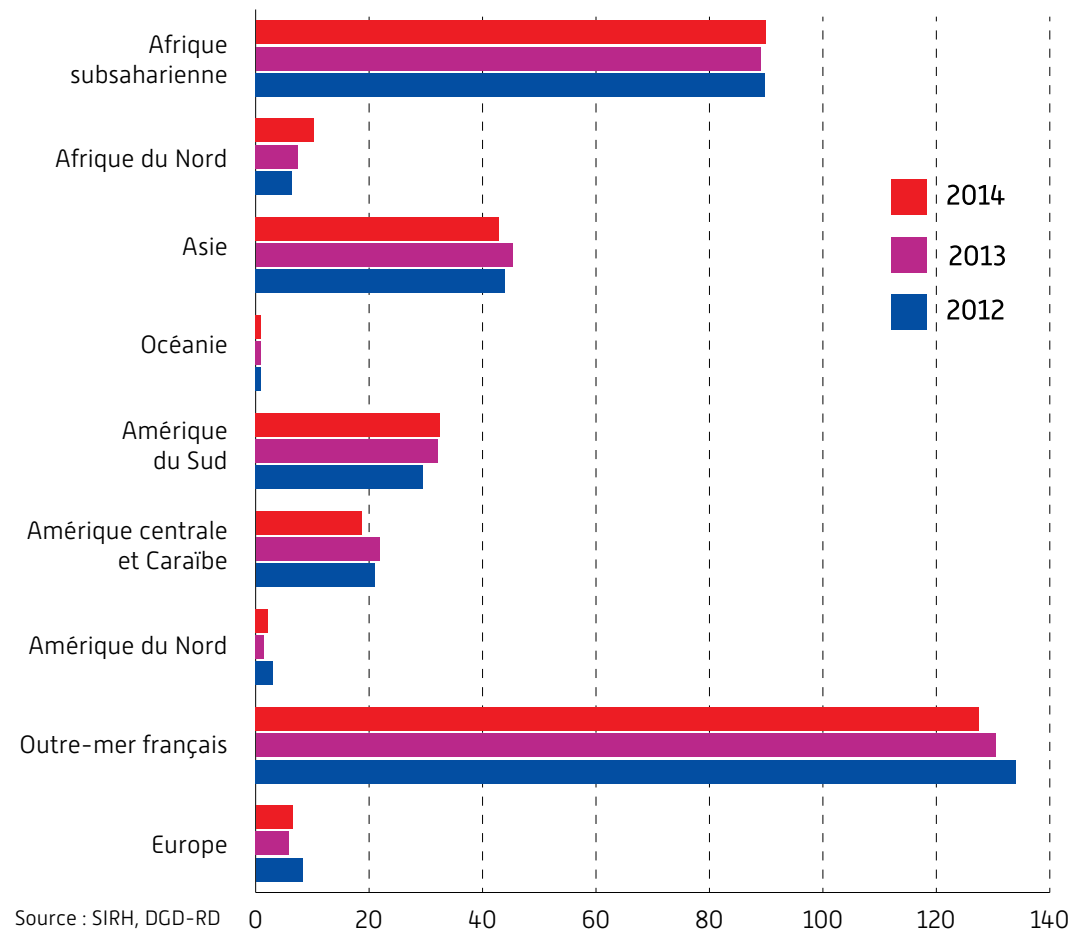

Nombre de cadres scientifiques affectés en mobilité géographique et répartition dans les dispositifs en partenariat (DP) et dans les DOM [en ETPT]

\begin{tabular}{lccc} 
& 2012 & 2013 & 2014 \\
\hline $\begin{array}{l}\text { Mobilité } \\
\text { géographique }\end{array}$ & 302 & 304 & 302 \\
\hline $\begin{array}{l}\text { Mobilité } \\
\text { en DP }\end{array}$ & 94 & 130 & 129 \\
\hline $\begin{array}{l}\text { Mobilité } \\
\text { dans les DOM }\end{array}$ & 113 & 113 & 114 \\
\hline
\end{tabular}

Source : SIRH, DGD-RD

Répartition des missions selon la destination [en \% d'équivalent temps plein]

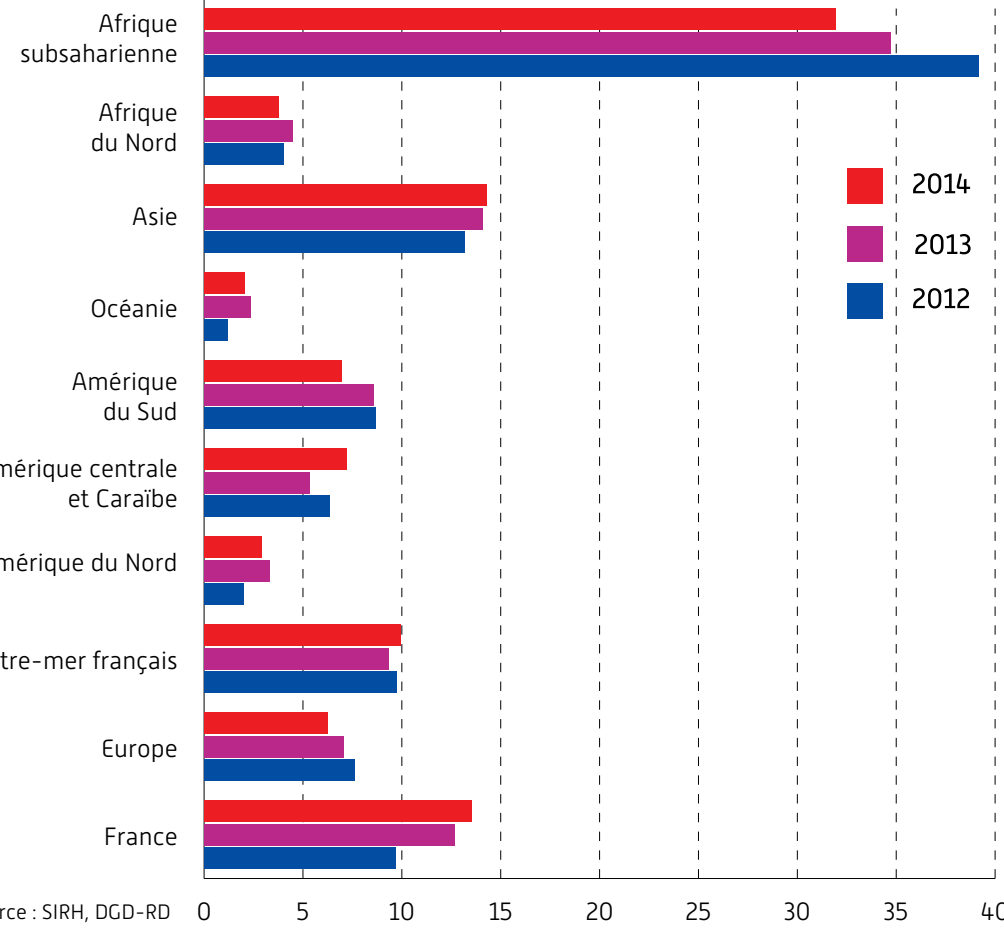




\section{Tableau des dispositifs de recherche et d'enseignement en partenariat [DP]}

\begin{tabular}{|c|c|c|}
\hline Région & DP nationaux & DP régionaux \\
\hline \multicolumn{3}{|c|}{ AFRIQUE } \\
\hline & PPZS - Sénégal- Axes 1, 5, 6 & ASAP - Afrique de l'Ouest - Axes 1, 4, 6 \\
\hline & RP-PCP - Zimbabwe - Axes 1, 4, 6 & DPFAC - Afrique centrale - Axes 1,6 \\
\hline & CRDPI - Congo - Axes 1, 6 & SISTO - Afrique de I'Ouest - Axes 3, 5, 6 \\
\hline & Forêt Biodiversité - Madagascar - Axes 2, 5, 6 & DIVECOSYS- Afrique de l'Ouest - Axe 1 \\
\hline \multicolumn{3}{|c|}{ PCP Agroforesterie - Cameroun - Axes 1, 5, 6} \\
\hline \multicolumn{3}{|c|}{ PP \& G - Afrique du Sud - Axes 5, 6} \\
\hline \multicolumn{3}{|c|}{ SPAD - Madagascar - Axes 1,6 } \\
\hline \multicolumn{3}{|l|}{ ASIE } \\
\hline & HRPP - Thaïlande - Axes 1, 6 & CANSEA - Asie du Sud-Est - Axes 1,6 \\
\hline & MALICA - Vietnam - Axes 3, 5 & GREASE - Asie du Sud-Est - Axe 4 \\
\hline \multicolumn{3}{|c|}{ AMÉRIQUE LATINE } \\
\hline & PCPAFS-PC - Costa Rica - Axes 1, 5, 6 & AMAZONIE - Bassin amazonien - Axe 6 \\
\hline \multirow{2}{*}{\multicolumn{2}{|c|}{ CIBA - Brésil - Axe 1}} & PP -AL - Amérique latine (10 pays) - Axes 5, 6 \\
\hline & & RéSA-CaribVET - Guadeloupe-Caraïbe - Axe 4 \\
\hline
\end{tabular}

Source : Délégation aux dispositifs en partenariat, DGD-RS

\section{Les projets européens de recherche et de développement (PCRDT) du Cirad entre 2010 et 2014}

\begin{tabular}{lccccc} 
& 2010 & 2011 & 2012 & 2013 & 2014 \\
\hline $\begin{array}{l}\text { Nombre de projets } \\
\text { soumis }\end{array}$ & 18 & 21 & 20 & 24 & 24 \\
\hline $\begin{array}{l}\text { Nombre de projets } \\
\text { financés }\end{array}$ & 7 & 5 & 8 & 7 & 3 \\
\hline $\begin{array}{l}\text { Taux de succès [\%] } \\
\text { Nombre de projets }\end{array}$ & 39 & 24 & 40 & 29 & 13 \\
$\begin{array}{l}\text { coordonnés par } \\
\text { le Cirad }\end{array}$ & 2 & 3 & 0 & 3 & 0 \\
\hline
\end{tabular}

Source : Délégation Europe, DCD-RS

\section{> UNE ORGANISATION ET DES MOYENS AJUSTÉS AUX NOUVEAUX DÉFIS}

En 2014, l'établissement a posé les bases d'un plan pluriannuel, le Pacte de développement des ressources, visant à consolider le modèle économique de l'établissement via des actions ciblées pour dynamiser son portefeuille de ressources contractuelles.

La très forte mobilisation des unités de recherche a permis dès 2014 d'accroître les ressources propres. Les indicateurs attestent une plus grande rentabilité de l'activité contractuelle après une période de baisse, avec notamment un accroissement des fonds européens (Fonds structurels et Fonds de R\&D]. Cependant, dans un contexte toujours contraint, la politique de maîtrise des dépenses a été maintenue en 2014.

Malgré une inflexion notable de la politique de l'emploi, l'emploi étant une priorité pour l'établissement, la tendance baissière des effectifs de l'établissement s'observe encore en 2014.
Dépenses d'exploitation hors sous-traitance interne 2010-2014, en millions d'euros

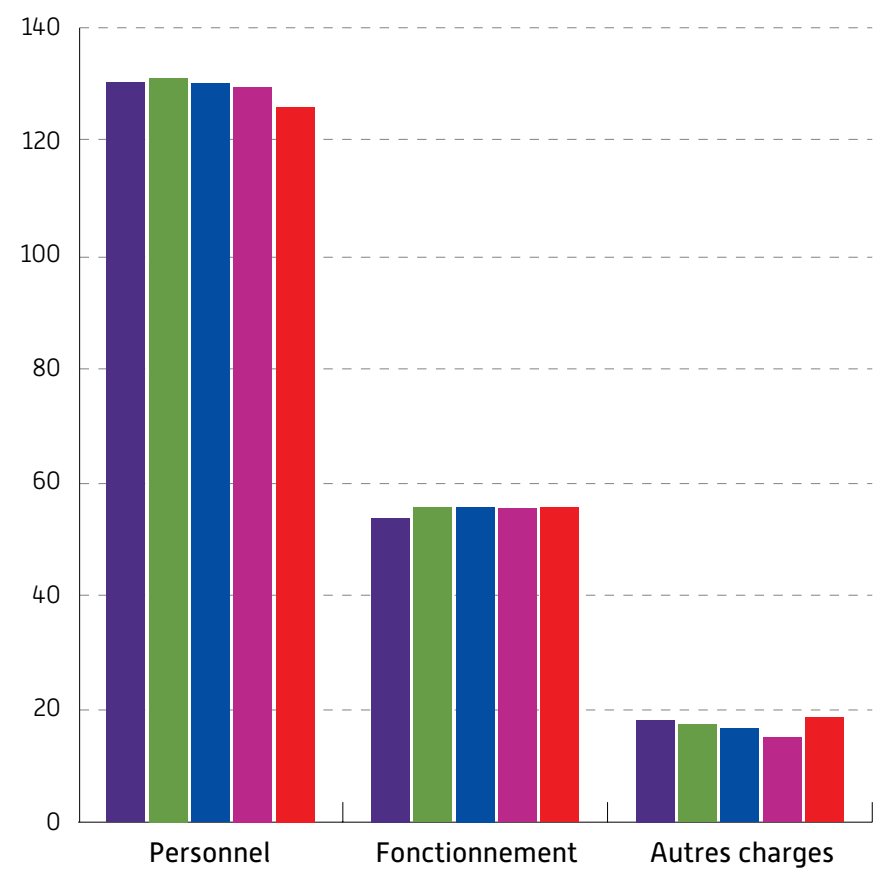

2010 
Ressources propres : volume et ventilation annuelle en pourcentage (hors cotraitance)

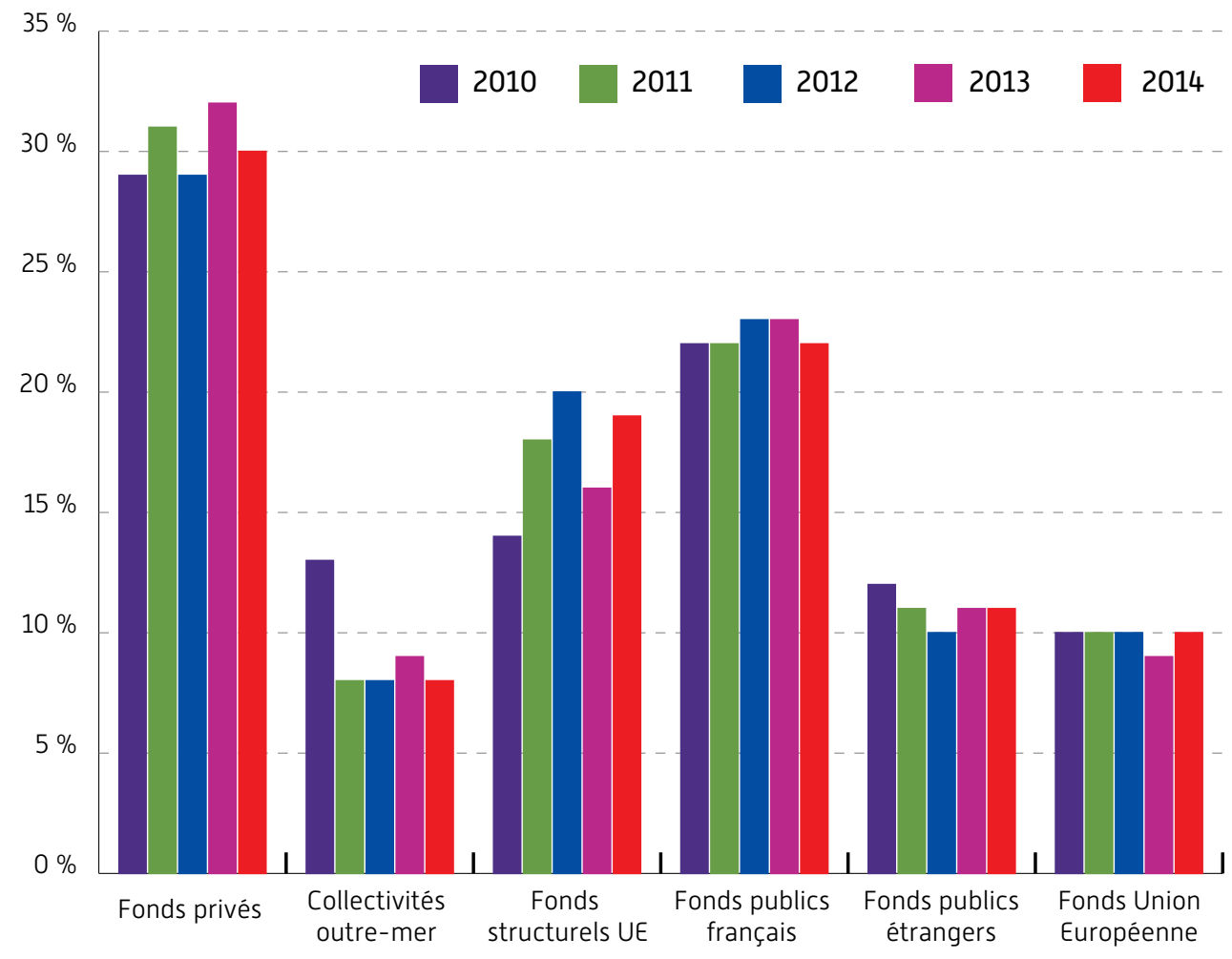

Source : Dcaf, DGD-RD [cf. « Rapport de gestion »)

Nombre total d'agents au Cirad [en équivalent temps plein]

\begin{tabular}{lcccccc} 
& $\mathbf{2 0 0 9}$ & $\mathbf{2 0 1 0}$ & $\mathbf{2 0 1 1}$ & $\mathbf{2 0 1 2}$ & $\mathbf{2 0 1 3}$ & $\mathbf{2 0 1 4}$ \\
\hline Nombre de « classés-payés » & 1764 & 1752 & 1739 & 1717 & 1681 & 1635 \\
\hline Nombre d'allocataires de thèses & 71 & 83 & 81 & 72,6 & 73,6 & 64
\end{tabular}

Source : SIRH-DGD-RD

Répartition annuelle des emplois " classés-payés " par catégories [incluant les allocataires de recherche sous contrat Cirad] (en pourcentage)

\begin{tabular}{lcccccc} 
& $\mathbf{2 0 0 9}$ & $\mathbf{2 0 1 0}$ & $\mathbf{2 0 1 1}$ & $\mathbf{2 0 1 2}$ & $\mathbf{2 0 1 3}$ & $\mathbf{2 0 1 4}$ \\
\hline Cadres & 59,2 & 59,6 & 60,6 & 62 & 62,9 & 64,1 \\
\hline Allocataires & 3,9 & 4,6 & 4,5 & 4,1 & 4,2 & 3,8 \\
\hline Maîtrise & 31,4 & 31,5 & 31,6 & 31,5 & 31 & 30,6 \\
\hline Collaborateurs & 5,5 & 4,4 & 3,4 & 2,4 & 1,8 & 1,5 \\
\hline
\end{tabular}

Source : SIRH-DGD-RD 
Rapport d'activités
Bilan et perspectives 201461

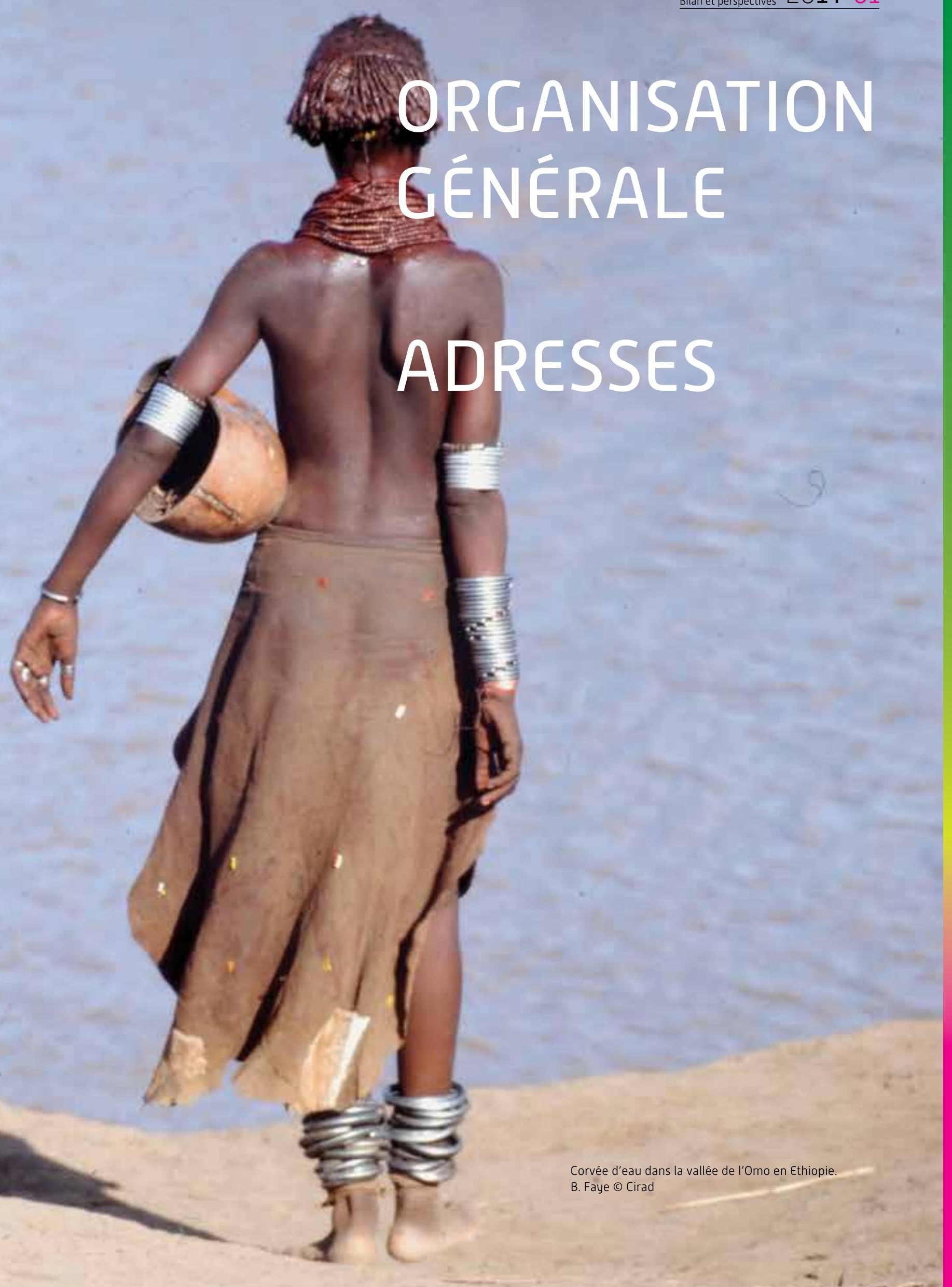




\section{Présence permanente des agents du Cirad dans le monde en 2014} (en équivalent temps plein travaillé - ETPT)

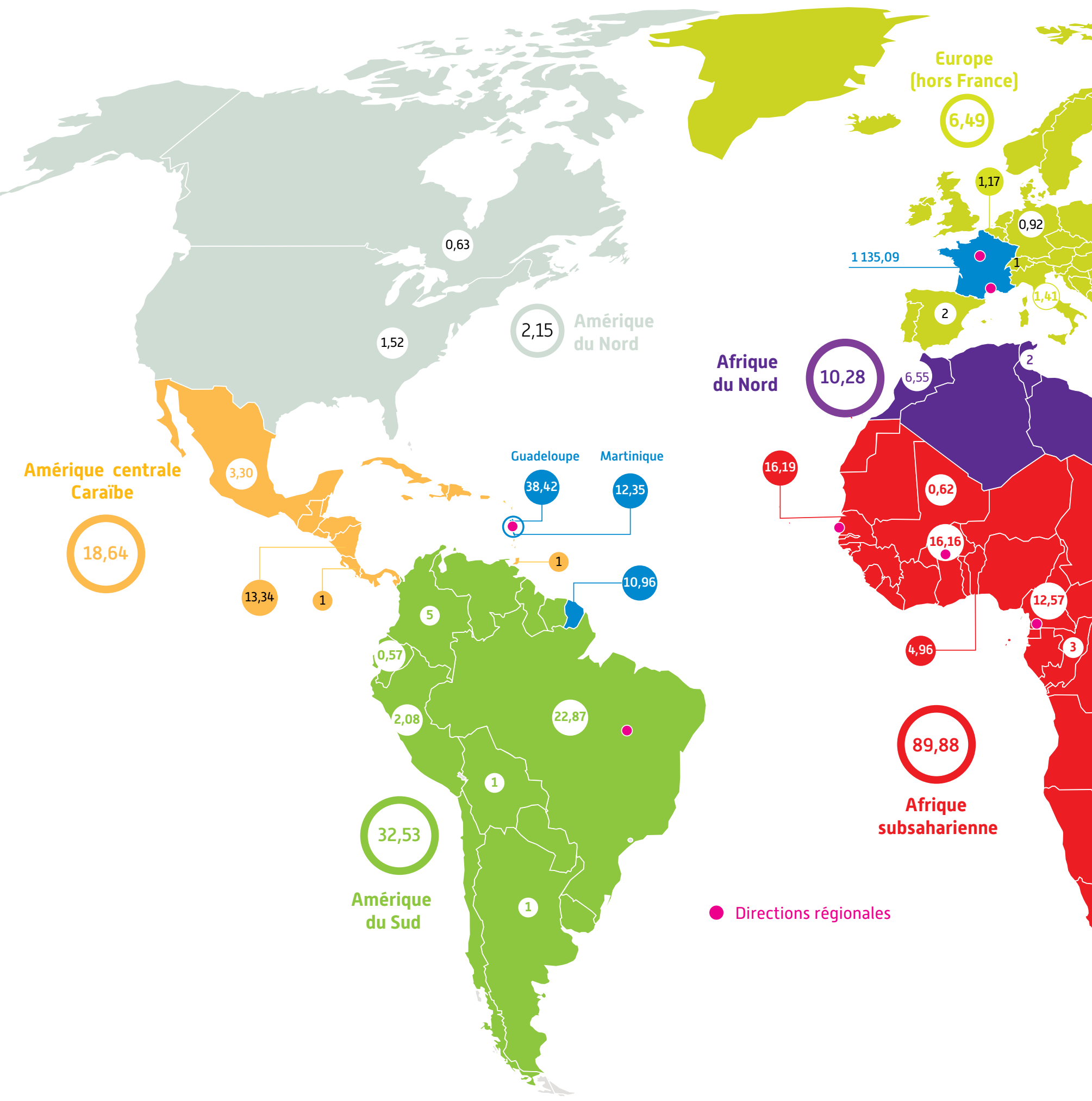




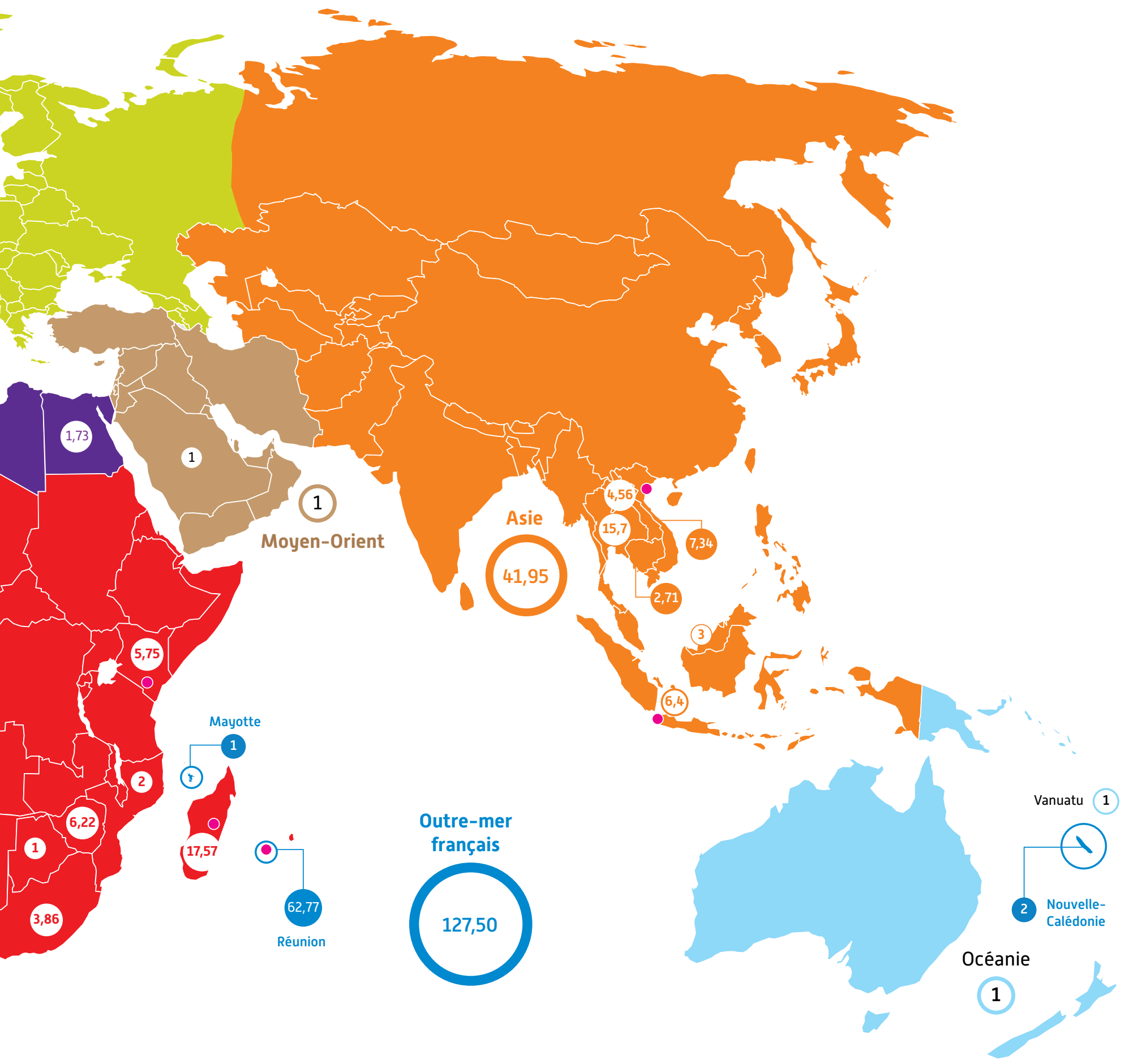


64 Rapport d'activités 2014

\section{Missions des agents du Cirad dans le monde en 2014}

(en équivalent temps plein travaillé - ETPT)

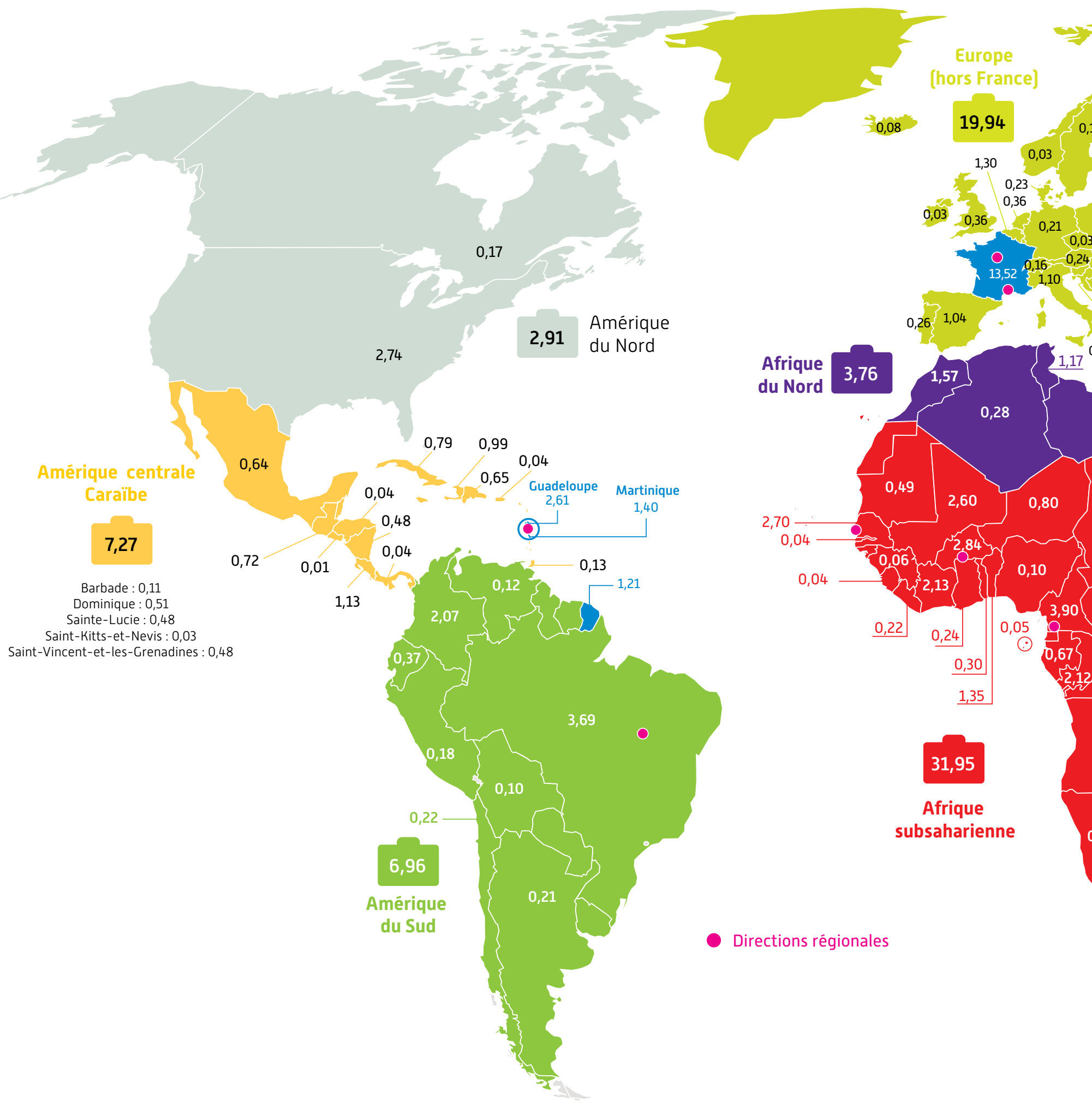




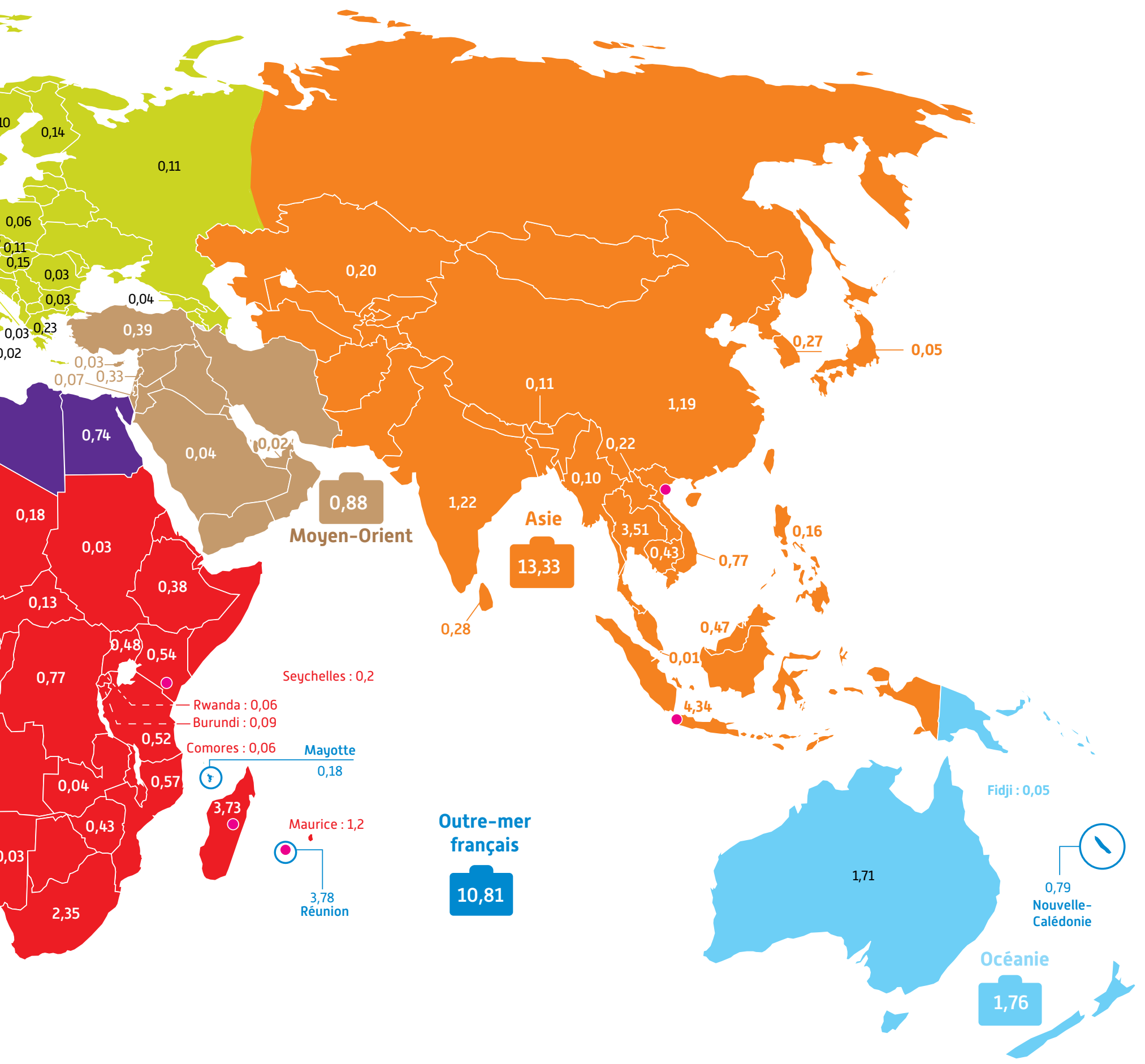




\section{Organisation générale du Cirad en avril 2015}

\author{
Conseil d'administration \\ Président \\ Michel Eddi

\begin{abstract}
M. Arnaud Martrenchar, ministère des Outre-mer

Mme Mireille Riou-Canals, ministère de de l'Agriculture,

Mme Corinne Brunon-Meunier, ministère des Affaires étrangères

M. Didier Hoffschir, ministère de de l'Enseignement supérieur et de la Recherche
\end{abstract} \\ de l'Agroalimentaire et de la Forêt
}

M. Charles Bélard,

ministère de l'Économie et des Finances

Gilles Boeuf, président du Muséum national d'histoire naturelle

Zoubida Charrouf, professeur à la faculté des Sciences de Rabat

François Houllier, président directeur général de l'Institut national de la recherche agronomique

Jean-Claude Moatti, président directeur général de l'Institut de recherche pour le développement

Isabelle Chmitelin, directrice de l'Office de développement de l'économie agricole des départements d'outre-mer

Laurence Tubiana, directrice de l'Institut du développement durable et des relations internationales

Les représentants élus du personnel François Affholder

Martine Antona

François Bousquet

Laurent Maggia

Jean-Louis Noyer

Philippe Vernier

Patrick Herbin secrétaire

Pierre-Luc Pugliese, rédacteur

\section{Conseil scientifıque \\ Membres nommés}

Président

Gilles Boeuf, président du Muséum national d'histoire naturelle

Alison Burrell, économiste

Bernard Chevassus-au-Louis, Inspecteur général de l'Agriculture
Laura Duarte, senior research associate à l'université de Brasilia

Louise Jackson, professeur botaniste écologue à UC Davis, California

Olivier Le Gall, directeur général délégué aux affaires scientifiques de l'Inra

Corinne Mence-Caster, présidente de l'université Antilles-Guyane

Harold Macauley, directeur général AfricaRice à Cotou, Bénin

Mohamed Sadiki, secrétaire général au ministère de l'Agriculture du Maroc

Marco Wopereis, directeur général adjoint AfricaRice à Cotonou, Bénin

\section{Membres élus}

Didier Bazile, agronome géographe François-Régis Goebel, entomologiste Jacques Imbernon, géographe Magalie Jannoyer, agrophysiologiste Eric Sabourin, socio-anthropologue

\section{Comité consultatif commun Inra-Cirad d'éthique pour la recherche agronomique}

Président

Louis Schweitzer,

Commissaire général à l'investissement

Fifi Benaboud, chercheur en sciences politique, coordinatrice du programme Transmed du Centre Nord-Sud du Conseil de l'Europe

Jean-Louis Bresson, médecin, professeur des universités au Centre d'Investigation Clinique

Marcel Bursztyn, socio-économiste, professeur à l'université de Brasilia, Centre pour le développement durable Paul Clavier, maître de conférences en philosophie à l'Ecole normale supérieure de Paris

Soraya Duboc, ingénieur agroalimentaire à Nestlé France

Françoise Gaill, directrice de recherche au Cnrs, chercheur dans les domaines des environnements profonds et à l'adaptation aux milieux extrêmes

Patrick du Jardin, professeur de biologie végétale à la faculté des Sciences agronomiques de Gembloux

Catherine Larrère, professeur d'éthique appliquée à l'université Paris I-Panthéon-Sorbonne
Sandra Laugier, professeur de philosophie à l'Université Paris 1 Jeanne-Marie Parly, professeur des universités en sciences économiques, Conseiller d'Etat

Hervé Théry, géographe, professeur associé à l'Universitē de São Paulo

Cérard Toulouse, directeur de recherche à l'Ecole normale supérieure de Paris Dominique Vermersch, agronome, professeur d'économie publique et d'éthique, Recteur de l'université Catholique de l'Ouest.

Pierre-Henri Duée (Inra) et Hubert Omont [Cirad], secrétaires

\section{Direction générale}

Michel Eddi,

président-directeur général

Etienne Hainzelin, conseiller

Patrick Herbin, conseiller

Anne Hébert, déléguée

à la communication

Marguerite Rodier-Goud, déléguée aux systèmes d'évaluation par interim

\section{Direction générale déléguée aux ressources}

\section{et aux dispositifs}

François Pouget, directeur

André Nau, directeur régional Ile-de-France

Michel Salas, directeur régional Languedoc-Roussillon

Jean-Luc Battini, directeur régional adjoint Languedoc-Roussillon

Dominique Martinez, directeur régional Antilles-Guyane

Gilles Mandret, directeur régional Réunion-Mayotte

Philippe Cao Van, adjoint au directeur régional Réunion-Mayotte

Marc Gélis, directeur de la comptabilité et des affaires financières

Sophie Beck-Gavelle, directeur adjoint de la comptabilité et des affaires financières, chargée des services comptable et financiers centraux
Brigitte Nesius, directeur adjoint de la comptabilité et des affaires financières, chargée des services financiers déconcentrés

Vincent Fabre-Rousseau, directeur des ressources humaines

Elisabeth Subirats, directeur adjoint des ressources humaines

Joël Sor, directeur des systèmes d'information

Myriam Valette, directeur technique des aménagements et de la maintenance

Léandre Mas, déléqué à la qualité et au développement durable

Thierry Corbineau, délégué aux affaires juridiques

André Nau, délégué au contrôle de gestion

Yann Combot, délégué aux archives

Patrice Guillaume, délégué à la santé et à la sécurité

Alexandre Polo, délégué aux achats Rémy Hugon, responsable de sécurité et de défense

\section{Direction générale déléguée à la recherche et à la stratégie}

Patrick Caron, directeur

Philippe Petithuguenin, directeur adjoint

Estelle Biénabe, adjointe au directeur Jean-Michel Sers, délégué pour l'Europe communautaire

Jacques Pagès, déléqué aux dispositifs en partenariat

Marie-Claude Deboin, déléguée à l'information scientifique et technique

Claudie Dreuil, déléguée aux actions incitatives

Cathy Grevesse, déléguée aux infrastructures de recherche

François Laporte, délégué à la valorisation et à l'innovation

Hubert Omont, délégué aux filières tropicales

Gilles Saint Martin déléqué aux chantiers stratégiques secrétaire du Conseil scientifique 


\section{Département Systèmes biologiques [Bios]}

Daniel Barthélémy, directeur

Dominique Berry, directeur adjoint

Jean-Louis Noyer, adjoint au directeur

\section{Unités de recherche*}

Amélioration génétique et adaptation des plantes méditerranéennes et tropicales (UMR Agap : Inra, Montpellier SupAgro),

Patrice This (Inra)

Bioagresseurs : analyse et maîtrise du risque [UPR],

Christian Cilas

Biologie et génétique des interactions plante-parasite (UMR BGPI : Inra, Montpellier SupAgro],

Claire Neema (Montpellier SupAgro]

Botanique et bio-informatique de l'architecture des plantes (UMR Amap : CNRS, université Montpellier 2 , Inra, IRD),

Thierry Fourcaud (Cirad)

Centre de biologie et gestion des populations (UMR CBGP : Inra, IRD, Montpellier SupAgro, Cirad], Flavie Vanlerberghe (Inra)

Contrôle des maladies animales exotiques et émergentes (UMR CMAEE : Inra), Thierry Lefrançois (Cirad)

Diversité, adaptation et développement des plantes (UMR Diade : IRD

Montpellier SupAgro, Inra, université Montpellier 2],

Alain Ghesquière (IRD)

Interactions hôtes-vecteursparasites-environnement dans les maladies tropicales négligées dues aux trypanosomatidés (UMR Intertryp : IRD), Philippe Solano [IRD]

Laboratoire des symbioses tropicales et méditerranéennes (UMR LSTM : université Montpellier 2, Inra, IRD, Montpellier SupAgro], Robin Duponnois [IRD]

Peuplements végétaux et bioagresseurs en milieu tropical (UMR PVBMT :

université de la Réunion),

Bernard Reynaud (Cirad)
Département Performances

des systèmes de production et de transformation tropicaux

\section{[Persyst]}

François-Xavier Côte, directeur

Hervé Saint Macary, directeur adjoint

Nadine Zakhia-Rozis, adjointe

au directeur

\section{Unitēs de recherche*}

Agroécologie et Intensification Durable des cultures Annuelles (UPR),

Eric Scopel

Analyses des eaux, sols et végétaux (US), Daniel Babre

Démarche intégrée pour l'obtention d'aliments de qualité (UMR Qualisud : universités Montpellier 1 et 2 ,

Montpellier SupAgro], Antoine Collignan [Montpellier SupAgro]

Ecologie fonctionnelle et biogéochimie des sols et des agroécosystèmes (UMR Eco-Sols : IRD,

Montpellier SupAgro, Inra], Jean-Luc Chotte (IRD)

Fonctionnement agroécologique et performances des systèmes $d$ e culture horticoles (UPR),

Eric Malézieux

Fonctionnement et conduite des systèmes de culture tropicaux et méditerranéens (UMR System : Inra, Montpellier SupAgro], Christian Gary (Inra)

Ingénierie des agropolymères et technologies émergentes (UMR late : université Montpellier 2, Inra, Montpellier SupAgro], Hugo de Vries (Inra)

Intensification raisonnée et écologique pour une pisciculture durable (UMR Intrépid : Ifremer), Béatrice Chatain (Ifremer)

Performance des systèmes de culture des plantes pérennes (UPR),

Eric Gohet

Production et valorisation des bois tropicaux, biomasse-énergie [UPR], Rémy Marchal

Recyclage et risque (UPR), Jean-Marie Paillat

Systèmes de culture bananiers plantains ananas (UPR), Jean-Michel Risède

\section{Département}

Environnements et sociétés

[ES]

Alain Billand, directeur

Sylvain Perret, directeur adjoint

Pascal Bonnet, adjoint au directeur

Hubert Devautour, adjoint au directeur

\section{Unités de recherche*}

Acteurs, ressources et territoires dans le développement [UMR Art-Dev : université Montpellier 3, CNRS), David Giband (université Montpellier 3] Animal et gestion intégrée des risques (UPR), François Roger

Biens et services des écosystèmes forestiers tropicaux [UPR],

Plinio Sist

Centre international de recherche sur I'environnement et le développement (UMR Cired : CNRS, EHESS,

AgroParisTech, École des Ponts-ParisTech),

Franck Lecocq (AgroParisTech)

Ecologie des forêts de Guyane (UMR Ecofog: AgroParisTech, Inra, CNRS, université des Antilles et de la Guyane), Eric Marcon (AgroParisTech)

Gestion de l'eau, acteurs et usages (UMR G-eau : Irstea, AgroParisTech, IAMM, IRD, Montpellier SupAgro], Olivier Barreteau [Irstea]

Gestion des ressources renouvelables et environnement [UPR], Martine Antona

Innovation et développement dans l'agriculture et l'agroalimentaire (UMR Innovation : Inra, Montpellier SupAgro],

Christophe Soulard (Inra)

Marchés, organisations, institutions et stratégies d'acteurs (UMR Moisa : CIHEAM-IAMM, Inra, Montpellier SupAgro],

Paule Moustier

Systèmes d'élevage méditerranéens et tropicaux (UMR Selmet: Montpellier SupAgro, Inra],

Alexandre Ickowicz

Territoires, environnement, télédétection et information spatiale (UMR Tetis : Irstea, AgroParisTech), Jean-Philippe Tonneau 


\section{Adresses}

\section{France}

Ile-de-France

André Nau,

directeur régional

42, rue Scheffer

75116 Paris

Tél. : +33153702021

andre.nau@cirad.fr

\section{Languedoc-Roussillon}

Michel Salas,

directeur régional

Avenue Agropolis

34398 Montpellier Cedex 5

Tél. : +33467615801

michel.salas@cirad.fr

\section{Antilles-Guyane}

Dominique Martinez,

directeur régional

Station de Neufchâteau, Sainte-Marie

97130 Capesterre-Belle-Eau,

Guadeloupe

Tél. : +590590861790 /

+0694451022

dominique.martinez@cirad.fr

Jean-Marc Thévenin,

correspondant

BP 701, avenue de France

97387 Kourou Cedex

Guyane

Tél. : +594594327352

jean-marc.thevenin@cirad.fr

Christian Chabrier,

représentant du Cirad en Martinique

BP 214

97285 Le Lamentin Cedex 2, Martinique

Tél. : +5965 96423044

christian.chabrier@cirad.fr

\section{Réunion-Mayotte}

Gilles Mandret, directeur régional

Station de La Bretagne, BP 20

97408 Saint-Denis Messageries Cedex 9

Réunion

Tél. : +262262528100 /

+262692763069

gilles.mandret@cirad.fr

\section{Autres localisations}

Yann Froelicher,

Correspondant en Corse

Centre Inra/Cirad

San Giuliano

20230 San Nicolao, France

Tél : +33495595911 /

+33495595959

yann.froelicher@cirad.fr

Laurent Maggia,

représentant

Centre IRD de Nouméa, BP 19239

98857 Nouméa Sud

Nouvelle-Calédonie

Tél : +687 260806

laurent.maggia@cirad.fr

\section{Afrique}

Afrique centrale

Patrice de Vernou,

directeur régional

Rue Joseph Essono Balla

BP 2572

Yaoundé, Cameroun

Tél. : +237222 212541

patrice.de_vernou@cirad.fr

Philippe Vigneron,

correspondant au Congo

BP 1291

Pointe-Noire,

République du Congo

Tél. : +24253563565

philippe.vigneron@cirad.fr

Afrique orientale et australe

Jacques Lançon,

directeur régional

C/o Icraf, United Nations Avenue

Gigiri, PO Box 30677

00100 Nairobi, Kenya

Tél. : +254207224653

jacques.lancon@cirad.fr

Mathieu Bourgarel,

correspondant au Zimbabwe

Cirad-Agirs

PO Box 1378

Harare, Zimbabwe

Tél. : +263775131601

mathieu.bourgarel@cirad.fr

\section{Afrique de l'Ouest continentale}

Patrice Grimaud,

directeur régional

688 Avenue du Professeur Ky-Zerbo

01 BP 596

Ouagadougou, BurkinaFaso

Tél. : +226 25307070

patrice.grimaud@cirad.fr

Philippe Menozzi,

correspondant au Bénin

Cirad/IRD

08 BP 841

31326 Cotonou, Bénin

Tél. : +22996725357

philippe.menozzi@cirad.f

\section{Afrique de l'Ouest côtière}

Denis Depommier,

directeur régional

37, avenue Jean XXIII

BP 6189

Dakar-Etoile, Sénégal

Tél. : +22133822 4484

denis.depommier@cirad.fr

\section{Madagascar}

Pascal Danthu

directeur régional

Ampandrianomby, BP 853

Antananarivo, Madagascar

Tél. : +2613207411 10

pascal.danthu@cirad.fr

\section{Amérique}

\section{Amérique centrale}

Fabrice Vaillant,

correspondant pour l'Amérique centrale

CITA - Universidad de Costa Rica

2060 San José, Costa Rica

Tél. : +506 25117210

fabrice.vaillant@cirad.fr

François Boucher

correspondant au Mexique

IICA - Calle San Francisco 1514

Colonia Tlacoquemecatl del Valle

03200 Mexico DF, Mexique

Tél. : +525555598519

francois.boucher@cirad.fr

Guy Henry

correspondant pour la Colombie,

l'Équateur et le Venezuela

Centro Internacional de Agricultura

Tropical

Cirad Alcue-KBBE Office - Ciat

KM 17 Recta Cali-Palmira, AA 6713,

Cali, Colombie

Tél. : +57244503124

guy.henry@cirad.fr

\section{Brésil}

Bernard Mallet,

directeur régional

SHIS-QI 16, Conj. 3, Casa 6

Lago Sul 71640-230

Brasilia DF, Brésil

Tél. : +55 6133661132

bernard.mallet@cirad.fr

\section{Asie}

Asie du Sud-Est continentale

Philippe Girard,

directeur régional

Cirad, Bureau 102, Bâtiment $2 \mathrm{G}$

Cité diplomatique de Van Phuc

$298 \mathrm{Kim} \mathrm{Ma}$

Hanoi, Vietnam

Tél. : +8443734 6775

philippe.girard@cirad.fr

Damien Jourdain,

correspondant en Thaïlande

Asian Institute of Technology

P.O.Box 4

Klong Luang Pathumthani Bangkok 12120, Thaïlande Tél. : +66 82657014 damien.jourdain@cirad.fr

Asie du Sud-Est insulaire

Alain Rival,

directeur régional

Graha Kapital 1

Jl. Kemang Raya no. 4

Jakarta 12730, Indonésie

Tél. : +62 217198641

alain.rival@cirad.fr

\section{Chine}

Zheng Li,

représentant permanent

Cirad-Inra

507 Tower A, Fuhua Mansion

8. Chaoyangmen North Avenue Beijing 100027, Chine Tél. : +86106554 1871 zhengliinra@sohu.com 
Directeur de publication : Michel Eddi, président du Conseil d'administration

Coordination générale : Patricia Doucet, délégation à la communication

Rédaction de la partie institutionnelle Faits marquants : Christine Nouaille

Rédaction de la partie Résultats de recherche : Frédéric Bourg, Caroline Dangléant, Laurence Dedieu, Patricia Doucet, Martine Lemaire, Hélène Petit

Rédaction de la partie Indicateurs : Estelle Bienabé

Diffusion et promotion : Christiane Jacquet

Conception graphique, infographie et mise en pages : Patricia Doucet

Illustration de couverture : Nathalie Le Gall

Impression : Impact Imprimerie, Saint-Gély-du-Fesc [34]

Imprimé avec des encres végétales sur papier entièrement recyclé (Cocoon Offset $90 \mathrm{~g}$ )

Le rapport d'activités du Cirad est également publié en anglais.

Dépôt légal : juin 2014 
cirad

LA RECHERCHE AGRONOMIQUE POUR LE DÉVELOPPEMENT

42, rue Scheffer

75116 Paris . France

Innovons ensemble pour

les agricultures de demain

www.cirad.fr 